Daniel Hermann

\author{
WANNIER-FunCTION BASED \\ SCATTERING-Matrix Formalism \\ for Photonic Crystal Circuitry
}





\title{
WANNIER-FunCtion BASED \\ SCATTERING-Matrix Formalism \\ for Photonic Crystal Circuitry
}

\author{
Zur Erlangung des akademischen Grades eines \\ DOKTORS DER NATURWISSENSCHAFTEN \\ von der Fakultät für Physik der \\ Universität Karlsruhe (TH) \\ genehmigte

\section{Dissertation}

von

Dipl.-Phys. Daniel Hermann aus Furtwangen

\footnotetext{
Tag der mündlichen Prüfung: 13.06 .2008

Referent:

Prof. Dr. K. Busch

Korreferent:

Prof. Dr. P. Wölfle
} 



\section{Contents}

$\begin{array}{lr}\text { Introduction } & 1\end{array}$

1. Fundamentals $\mathbf{5}$

1.1. Wave Propagation in Dielectric Media . . . . . . . . . . . . 5

1.1.1. Maxwell's Equations and Wave Equations . . . . . . . . . 5

1.1.2. Wave Equations in 2D Structures . . . . . . . . . . . 6

1.2. Photonic Crystals . . . . . . . . . . . . . . . . 7

1.2.1. Basic Concepts from Solid-State Theory . . . . . . . . . . . 8

1.2.2. Band Structure and Band Gaps . . . . . . . . . . . . . . . 10

1.2.3. Bloch Modes and Wannier Functions . . . . . . . . . . . . . 12

1.2.4. Wannier Function Generation . . . . . . . . . . . . . . 13

1.2.5. Single-Pore Infiltration . . . . . . . . . . . . . . . . . 15

1.3. Conventional Circuits . . . . . . . . . . . . . . . . 16

1.3.1. Directional Coupler . . . . . . . . . . . . . . . 16

1.3.2. Mach-Zehnder interferometer . . . . . . . . . . . . 18

2. Wannier Function Expansion $\quad 21$

2.1. Defect Structures in Photonic Crystals . . . . . . . . . . . . . . . 21

2.1.1. Point Defects . . . . . . . . . . . . . . . 22

2.2. Wave Equations in Wannier Basis . . . . . . . . . . . . . . . 23

2.2.1. 2D H-polarization . . . . . . . . . . . . . . 24

2.2.2. 2D E-polarization . . . . . . . . . . . . . . 25

2.2.3. 3D wave equations . . . . . . . . . . . . 25

2.2.4. Splitting into Point Defect Contributions . . . . . . . . 26

2.2.5. Properties of Overlap Matrices . . . . . . . . . . . . . 26

2.3. Classes of Defect Structures . . . . . . . . . . . . . . . . . 27

2.3.1. Calculation of Cavity Modes . . . . . . . . . . . 28

2.3.2. Calculation of Waveguide Modes . . . . . . . . . . . . . . 29

2.3.3. Calculation of the S-matrix of Functional Elements . . . . . . . 30

2.3.4. Optimization of Functional Elements . . . . . . . . . . . . 31

$\begin{array}{lr}\text { 3. Scattering Matrix Formalism } & \mathbf{3 5}\end{array}$

3.1. Basic Concept . . . . . . . . . . . . . . . . . 35

3.2. S-Matrix of Individual Functional Elements . . . . . . . . . . . . 36

3.2.1. Reduced S-Matrix . . . . . . . . . . . . . . . . 39

3.3. Symmetries . . . . . . . . . . . . . . . . 40 
3.3.1. Reciprocity . . . . . . . . . . . . . . . . . 40

3.3.2. Energy conservation . . . . . . . . . . . . . . . 42

3.3.3. Geometric Symmetries . . . . . . . . . . . . . . . . . . 42

3.3.4. Congruence of Functional Elements . . . . . . . . . . . . . . . 44

3.4. S-Matrix Combination . . . . . . . . . . . . . . . . 45

$\begin{array}{lr}\text { 4. Accuracy and Convergence } & 49\end{array}$

4.1. Model Systems . . . . . . . . . . . . . . . . . . . . . . 49

4.1.1. Auxiliary Wannier Functions . . . . . . . . . . . . . . . 50

4.2. Cavity Modes . . . . . . . . . . . . . . . . . . 52

4.2.1. Model System $1 \ldots \ldots \ldots$. . . . . . . . . . . 53

4.2.2. Model System $2 \ldots \ldots \ldots$. . . . . . . . . . . . . . . . . . . . . . 57

4.3. Waveguide Dispersion . . . . . . . . . . . . . . 60

4.3.1. Model System 1 . . . . . . . . . . . . . . 60

4.3.2. Model System 2 . . . . . . . . . . . . . . 63

4.4. Waveguide as a Functional Element . . . . . . . . . . . . 66

4.5. Double Bend . . . . . . . . . . . . . . . . . . 67

4.6. Simple Mach-Zehnder Interferometer . . . . . . . . . . . . . . . 69

5. Design of Large-Scale Circuits in a 2D Photonic Crystal 73

5.1. Basic Components . . . . . . . . . . . . . . . . . 73

5.1.1. Default Waveguide . . . . . . . . . . . . . 73

5.1.2. Optimized Bend . . . . . . . . . . . . . . . . 74

5.2. Directional Coupler . . . . . . . . . . . . . . . 76

5.2.1. Additional Components . . . . . . . . . . . . . 77

5.2.2. Length-Dependent Performance and Spectrum . . . . . . . . 80

5.3. Mach-Zehnder Interferometer . . . . . . . . . . . . . . . . . 82

5.3.1. Additional Components . . . . . . . . . . . . . . . . 82

5.3.2. Balanced and Unbalanced MZI . . . . . . . . . . . . . . . 84

5.4. Actively Tunable Mach-Zehnder Interferometer . . . . . . . . . . . . 88

5.4.1. Additional Components . . . . . . . . . . . . . . 88

5.4.2. Application: Switch or Modulator . . . . . . . . . . . . . 90

$\begin{array}{ll}\text { Summary and Conclusions } & 93\end{array}$

$\begin{array}{lr}\text { A. Model System } 1 & 97\end{array}$

$\begin{array}{ll}\text { B. Model System } 2 & 101\end{array}$

$\begin{array}{ll}\text { Acronyms } & 105\end{array}$

$\begin{array}{ll}\text { Bibliography } & 107\end{array}$ 


\section{Introduction}

Lately, we celebrated the 15th anniversary of the World Wide Web. On April 30 1993, the $\mathrm{CERN}^{1}$ put this technology for internet content in the public domain [1] and, thereby, started the rapid growth of the internet. Nowadays, about 1.3 billion people [2] worldwide use the internet as a source of information and a communication media. Terabytes of data are sent all around the globe every second. The main foundation for such ever increasing data rates has been provided by optical fiber technology. Optical fibers are able to guide light over large distances with little propagation loss. They provide a much larger bandwidth than electrical connections due to their operation at higher frequencies, typically in the short-wavelength infrared (around $1.5 \mu \mathrm{m}, 200 \mathrm{THz})$.

While optical communication clearly outperforms electronics for signal transport, it is considerably more difficult to process signals all-optically and to build up all-optical logical circuits. Up to now, routing in optical fiber networks, for instance, is still done with electronics. For that, the light is converted to an electronic signal, is then processed by some electronic circuitry, and is resubmitted to an optical fiber. This slows down the network, narrows its bandwidth and, moreover, is power-intensive. The electronic components tend to be the bottleneck of the communication pipeline. Clearly, new concepts and technologies for all-optical signal processing are needed.

One step in this direction is the development of on-chip photonic integrated circuits $[3,4]$. Planar waveguiding structures on a substrate provide the basis for complex functionality. Several materials and technologies are used, such as Silica-on-Silicon (similar to a planar realization of an optical fiber) [5], Silicon-on-Insulator (SOI) $[6,7]$ or III-V semiconductor structures. The latter are favorable for the simultaneous integration of active components such as laser diodes and photodetectors to create and detect light on-chip. Depending on the refractive-index contrast in the waveguiding structures, those technologies allow to scale down the size of fabricated circuits. While Silica-on-Silicon waveguides have a small index contrast (less than $1 \%$ between core and cladding, both made of silica) and sizes of tens of micrometers, high-contrast SOI Photonic Wires (silicon waveguides surrounded by air on a silica cladding, index contrast $3.45: 1.0: 1.45$ ) have dimensions of $500 \mathrm{~nm}$ or less.

A serious limitation for the size reduction of photonic integrated circuits is the loss of guidance at waveguide bends. If light is guided by total internal reflection, it can escape as soon as it hits the waveguide core interface in an angle larger than the critical angle. For low-contrast waveguides (optical fibers, Silica-on-Silicon waveguides), this effect sets in for bend radii of $1 \mathrm{~cm}$ and smaller. High-contrast Photonic

\footnotetext{
${ }^{1}$ European Organization for Nuclear Research
} 
Wires exhibit bend losses, too, albeit the bends can have radii down to a few $\mu \mathrm{m}$ $[8,9]$ before bend losses are significant. Nevertheless, all index-guided structures suffer from the fact that the waveguides are not surrounded by a photonic insulator, which would prohibit the propagation of the light outside the waveguide. Instead, the surrounding air or low-index material is perfectly conducting for light.

A solution to this problem is the use of Photonic Crystals (PhCs) [11-14] as host materials for photonic integrated circuits. This class of artificial materials is characterized by a periodically varying dielectric permittivity with a lattice period of the same order of magnitude as the wavelength of light. Analogous to the scattering of electrons at the crystal potential of an "electronic" crystal, scattering of light at the periodic dielectric structure of the $\mathrm{PhC}$ leads to the formation of a band structure in the frequency spectrum. Properly designed, a $\mathrm{PhC}$ can exhibit photonic band gaps (PBGs), i.e., frequency ranges, in which propagation of light is completely pro-

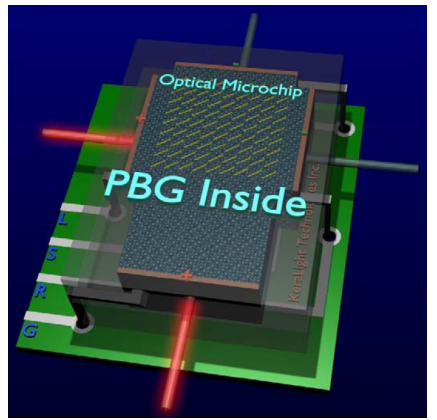

all-optical microchip [10] hibited. For frequencies in the band gap, the PhC represents a perfect photonic insulator, analogous to a semiconductor for electrons. Therefore, it is possible to create sharp bends within such a PhC by introducing defects (analogous to the "doping" of semiconductors) and to overcome the limitations of index-guided structures.

Besides the existence of band gaps, the unusual and unique dispersion properties of $\mathrm{PhCs}$ and $\mathrm{PhC}$ waveguides give rise to many other applications. Prominent examples are the slow light propagation near band edges or in flat bands [15-17], the superrefractivity (superprism effect) [18], and the negative refraction (superlenses) $[19,20]$. Furthermore, PhCs offer the possibility to investigate fundamental problems in quantum optics $[21,22]$ and condensed matter physics. Independent of each other in 1987, Eli Yablonovitch and Sajeev John initially proposed the use of PhCs in the context of the inhibition of spontaneous emission [23] and of the strong localization of photons [24], respectively. Today, they are even used in quantum-information processing applications [25]. The deliberate creation of a "designer vacuum" with the desired density of states for photons is an attractive vision for both theorists and experimentalists.

Soon after the theoretical proposal of PhCs, experimentalists and material scientists started to investigate different techniques and materials to build prototypes. Beyond the creation of one-dimensional (1D) PhCs, better known as Bragg stacks, which exhibit periodicity in only one dimension and are homogeneous in the other directions, considerable efforts have been undertaken to experimentally realize $\mathrm{PhCs}$ with periodicity in two [26-30] and three [31-41] dimensions (2D and 3D PhCs). With respect to photonic integrated circuits, 2D silicon $\mathrm{PhCs}[28]$ and $\mathrm{PhC}$ slabs $[42,43]$ are of particular interest. High-quality 2D crystals with a large extent in the homogeneous $z$-direction have been produced by electrochemically growing nanoscale pores in n-type silicon $[44,45]$. Such high index-contrast macroporous silicon 2D PhCs can have large band gaps for the propagation of light in the plane of periodicity. However, 
the majority of $\mathrm{PhC}$ structures that have been produced and characterized are $\mathrm{PhC}$ slabs, in which the extent in the "homogeneous" $z$-direction is comparable or even smaller than the lattice constant in the plane. In these structures, guidance with respect to the $z$-direction is achieved only by total internal reflection. Furthermore, $\mathrm{PhC}$ slabs with complete band gaps in the plane are rare [46]. Nevertheless, besides well-established techniques such as electron-beam lithography, existing CMOS technology can be used in the production of $\mathrm{PhC}$ slab-based photonic integrated circuits [4], which makes this option very attractive for industrial realization.

Simultaneously with the development of material and manufacturing technologies, extensive studies about different types of functional elements built into PhCs have been carried out over the past decade. The potential for broadband single-mode waveguides [47] and bends [48-50] as well as for more complex devices, such as directional couplers [51, 52] and Mach-Zehnder interferometers [53, 54] has been demonstrated. In addition, various concepts for active tunability using liquid crystals have been proposed [55-58].

In the face of the great design freedoms and the complexity of $\mathrm{PhC}$ structures, it is of utmost importance to have powerful theoretical and computational tools to characterize, simulate and optimize such structures. The plane wave expansion method (PWM) [59-63] is widely used to calculate band structures for bulk PhCs. By using supercells, it can also be utilized for cavity mode and waveguide dispersion calculations. Alternatively, powerful real-space methods [64, 65] can be utilized for band structure calculations. For non-periodic structures, by far the most simulations have been done using the Finite-Difference Time-Domain (FDTD) method [66] or other all-purpose techniques, such as Finite Element [67] or Beam Propagation methods [68]. While these methods are very powerful for structures with an arbitrary distribution of dielectric material, they lose some efficiency in highly ordered structures such as PhCs since they do not exploit the symmetry properties and band structure of the underlying PhC. Furthermore, the simulation of large-scale devices is very memory- and/or time-consuming, especially in the case when slow light regimes [53] are involved in one of the components.

In this thesis, I develop and investigate in detail a scattering-matrix approach for the efficient treatment of $\mathrm{PhC}$ circuits [69] and demonstrate its applicability to largescale $^{2} 2 \mathrm{D}$ circuits. The approach relies on the efficient calculation of the scattering matrices of basic functional elements using an expansion of fields into photonic Wannier functions [70], which represent a basis ideally suited to describe localized fields within defect structures in $\mathrm{PhCs}$.

This thesis is structured as follows. In chapter 1, I introduce the general concept of photonic crystals and give an overview about band structures, Bloch modes and Wannier functions. In addition, I introduce the working principles of some basic conventional circuits such as directional couplers and Mach-Zehnder interferometers.

Chapters 2 and 3 are devoted to the detailed description of the Wannier-function

\footnotetext{
${ }^{2}$ In this context, "large-scale" means large compared to the lattice constant of the $\mathrm{PhC}$, which lies in the sub-micron regime, typically about $0.5 \mu \mathrm{m}$.
} 
based scattering-matrix approach. In chapter 2, I provide technical details about the Wannier function expansion. The wave equation is formulated in the Wannier function basis and matrix equations for the calculation of cavity modes, waveguide guided modes and S-matrices of functional elements are derived. I briefly discuss the optimization of functional elements. In chapter 3 , I outline the scattering-matrix formalism. On the one hand, I explain the calculation of individual functional elements on the "microscopic" level using Wannier functions. On the other hand, I discuss symmetries of S-matrices and the impact of congruences of functional elements and formulate the combination of individual S-matrices to the circuit S-matrix.

In the last two chapters, I apply the method in two different ways. In chapter 4, the focus is put on testing the method for accuracy and convergence. To do this, I present Wannier function calculations of cavity modes and waveguide dispersions and compare the results to reference solutions computed with the plane-wave based MIT photonic bands package (MPB) [63]. Furthermore, checks of the self-consistency of the scattering-matrix combination are carried out. In contrast to this, in chapter 5 , I demonstrate the feasibility and full power of the scattering-matrix method on the basis of the design of an actively tunable Mach-Zehnder interferometer. The process involves the design of basic components such as an optimized bend or a tunable delay line using liquid-crystal infiltration.

Finally, a summary and conclusions are provided. 


\section{Fundamentals}

\subsection{Wave Propagation in Dielectric Media}

\subsubsection{Maxwell's Equations and Wave Equations}

Electromagnetic wave propagation in arbitrary media is governed by the Maxwell equations

$$
\begin{aligned}
\nabla \cdot \vec{D} & =\rho & \nabla \cdot \vec{B} & =0 \\
\nabla \times \vec{E} & =-\frac{\partial}{\partial t} \vec{B} & \nabla \times \vec{H} & =\vec{j}+\frac{\partial}{\partial t} \vec{D}
\end{aligned}
$$

with the electric and magnetic field $\vec{E}(\vec{r}, t)$ and $\vec{H}(\vec{r}, t)$ and the electric and magnetic flux density $\vec{D}(\vec{r}, t)$ and $\vec{B}(\vec{r}, t)$, respectively. These equations are generally applied to macroscopic averages of the fields since on a microscopic scale the fields vary strongly due to the inhomogeneous charge distribution in the medium. In the macroscopic picture, the influence of matter is described by a polarization density $\vec{P}$ and a magnetization density $\vec{M},{ }^{1}$ which enter Maxwell's equations through so-called constitutive relations

$$
\begin{aligned}
\vec{D} & =\epsilon_{0} \vec{E}+\vec{P}(\vec{E}, \vec{H}), \\
\vec{B} & =\mu_{0} \vec{H}+\vec{M}(\vec{E}, \vec{H})
\end{aligned}
$$

that connect the flux densities $\vec{D}$ and $\vec{B}$ to the fields $\vec{E}$ and $\vec{H}$.

In general, $\vec{P}(\vec{E}, \vec{H})$ and $\vec{M}(\vec{E}, \vec{H})$ are complicated functionals of $\vec{E}$ and $\vec{H}$, covering non-local, non-linear and non-Markovian effects. This gives rise to a rich variety of optical phenomena observed in various media.

In this thesis, however, we restrict ourselves to the case of non-magnetic ${ }^{2}$ materials with linear, local, instantaneous response. In this case, we have

$$
\begin{aligned}
\vec{P} & =\epsilon_{0} \chi_{e} \vec{E} & \vec{M} & =0 \\
\vec{D} & =\epsilon_{0}\left(1+\chi_{e}\right) \vec{E}=\epsilon_{0} \epsilon \vec{E} & \vec{B} & =\mu_{0} \vec{H} .
\end{aligned}
$$

\footnotetext{
${ }^{1}$ Higher moment densities, such as electric quadrupoles, are completely negligible in most materials [71]. Therefore, they are left out in our considerations.

${ }^{2}$ Most conventional materials are non-magnetic at optical frequencies [71]. However, there currently is a huge interest in constructing artificial materials (meta-materials) with magnetic response at optical frequencies. This would allow to realize negative refractive index materials $[72,73]$ for perfect lenses [74] and cloaking devices [75], for instance.
} 
The material response is described by the frequency-independent dielectric permittivity $\epsilon=\epsilon(\vec{r})$, which, in general, is a tensor. In most parts of this thesis, however, we will treat $\epsilon(\vec{r})$ to be a scalar unless otherwise specified ${ }^{3}$. We further assume that there exist no free charges $(\rho=0)$ and currents $(\vec{j}=0)$ in the materials.

By multiplying the curl equation (1.2) for $\vec{H}$ with $\epsilon^{-1}(\vec{r})$ and again applying curl to it, we can derive the electromagnetic wave equation for the $\vec{H}$-field

$$
\nabla \times \frac{1}{\epsilon(\vec{r})} \nabla \times \vec{H}(\vec{r}, t)+\frac{1}{c^{2}} \frac{\partial^{2}}{\partial t^{2}} \vec{H}(\vec{r}, t)=0 .
$$

The wave equation for the $\vec{E}$-field,

$$
\frac{1}{\epsilon(\vec{r})} \nabla \times \nabla \times \vec{E}(\vec{r}, t)+\frac{1}{c^{2}} \frac{\partial^{2}}{\partial t^{2}} \vec{E}(\vec{r}, t)=0,
$$

can be derived similarly. In frequency domain, these equations read

$$
\begin{aligned}
& \nabla \times \frac{1}{\epsilon(\vec{r})} \nabla \times \vec{H}(\vec{r})-\frac{\omega^{2}}{c^{2}} \vec{H}(\vec{r})=0 \\
& \frac{1}{\epsilon(\vec{r})} \nabla \times \nabla \times \vec{E}(\vec{r})-\frac{\omega^{2}}{c^{2}} \vec{E}(\vec{r})=0 .
\end{aligned}
$$

The major task is to develop methods for solving the wave equations for a given dielectric permittivity $\epsilon(\vec{r})$ under given boundary conditions. In band structure calculations, for instance, (1.9) is solved as a standard eigenvalue problem for the eigenvalues $\omega^{2} / c^{2}$ with Bloch boundary conditions. For the calculation of scattering matrices of functional elements, on the other hand, the frequency is fixed and (1.9) is solved as a linear system of equations with mixed boundary conditions (see section 2.3.3).

\subsubsection{Wave Equations in 2D Structures}

For arbitrary 3D structures the wave equations (1.9) and (1.10) have to be solved as fully vectorial problems. However, in the case of structures that are homogeneous in one direction ( $\hat{z}$-direction) and for propagation in the $x-y$ plane it is possible to decouple the two different polarizations, the $H$-polarization $(\vec{H}$-field parallel to $\hat{z})$ and the $E$-polarization $(\vec{E}$-field parallel to $\hat{z}) .{ }^{4}$ The resulting scalar wave equations for the $z$-components of the fields read

$$
\begin{array}{rlr}
{\left[\nabla_{2} \cdot \frac{1}{\epsilon(\vec{r})} \nabla_{2}\right] H(\vec{r})+\frac{\omega^{2}}{c^{2}} H(\vec{r})} & =0 & (H \text {-polarization }) \\
\frac{1}{\epsilon(\vec{r})} \Delta_{2} E(\vec{r})+\frac{\omega^{2}}{c^{2}} E(\vec{r}) & =0 & (E \text {-polarization })
\end{array}
$$

\footnotetext{
${ }^{3}$ The generalization to dielectric permittivity tensors is done in detail in [76]

${ }^{4}$ Often the terms TE- (transverse electric) and TM-polarization (transverse magnetic) are used in the literature instead of $H$ - and $E$-polarization, respectively. This nomenclature is sometimes ambiguous, therefore we don't use it here.
} 


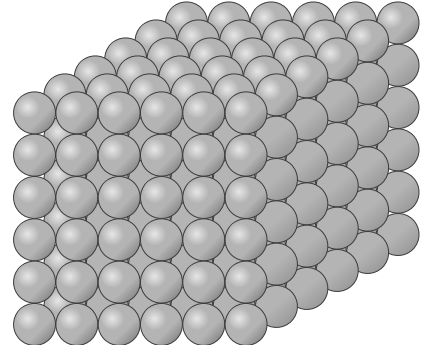

(a) $3 \mathrm{D}$

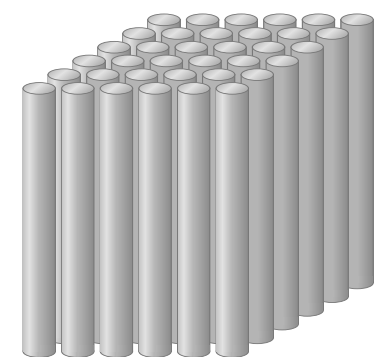

(b) $2 \mathrm{D}$

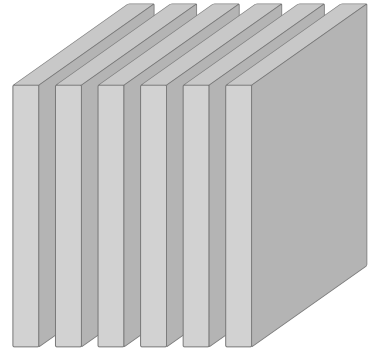

(c) $1 \mathrm{D}$

Figure 1.1.: Schematic pictures of PhCs of different dimensionality. The continuous translational symmetry is retained in (a) no, (b) one, or (c) two directions.

with the definitions

$$
\nabla_{2} \equiv\left(\begin{array}{c}
\partial_{x} \\
\partial_{y}
\end{array}\right) \quad \Delta_{2} \equiv \partial_{x}^{2}+\partial_{y}^{2} .
$$

Since we can treat the two polarizations separately and the equations are scalar, this greatly simplifies the problem. The solutions of (1.11) and (1.12) still cover the entire space of solutions of the fully $3 \mathrm{D}$ wave equations for the $2 \mathrm{D}$ structures.

For systems structured in the $x-y$-plane and homogeneous but finite in the $z$ direction (slab structures), the above $2 \mathrm{D}$ description is no longer valid since the two polarizations couple. Nevertheless, results obtained by $2 \mathrm{D}$ calculations can still be useful for qualitative discussions as the modes found in slab structures typically show significant similarities to $H$ - or $E$-polarized modes of the strictly $2 \mathrm{D}$ calculation. In many cases they can be categorized as "TE-like" or "TM-like". We will not discuss this issue in this thesis and refer the reader to the literature [42, 43] for more details.

\subsection{Photonic Crystals}

Photonic Crystals (PhCs) [11-13] are periodically structured dielectric materials with the spatial period on the order of the wavelength of light. They may consist of a periodic arrangement of dielectric "atoms" (e.g. dielectric spheres) or the inverse structure (e.g. air spheres in dielectric material) and can be classified by dimensionality. While 3D crystals exhibit a discrete translation symmetry in all three directions of space, 2D crystals have a periodic structure in a plane but are homogeneous in the third direction. 1D crystals have only one direction of discrete translational symmetry and are homogeneous in planes perpendicular to that direction. A schematic picture of all three cases is given in Fig. 1.1.

Electromagnetic waves are scattered at interfaces between materials of different dielectric permittivity. If these interfaces are arranged periodically, the situation is analogous to electronic crystals where scattering of the electron wave function on the effective crystal potential leads to the formation of an electronic band structure in 
the energy spectrum. Therefore, we expect similar phenomena to appear in Photonic Crystals. In particular, we expect a photonic band structure in the frequency spectrum of the electromagnetic waves and the occurrence of band gaps, i.e. regions of frequency where electromagnetic wave propagation is prohibited in the Photonic Crystal. On the other hand, there also exist fundamental differences between electronic and photonic wave propagation:

- the electromagnetic field has vectorial nature,

- the photon energy (frequency) is always positive,

- the photonic crystal "potential" is frequency-dependent and vanishes for $\omega \rightarrow 0$.

Due to the vectorial nature it is, for instance, more difficult to find structures with complete photonic band gaps. The positive photon energy and the vanishing "crystal potential" for $\omega \rightarrow 0$ make it more difficult to achieve localization of photons.

The existence of band gaps is the most prominent feature of Photonic Crystals (also frequently called "Photonic Band Gap Materials", PBGs). It is the precondition for the $\mathrm{PhC}$ cavities, waveguides and defect structures discussed later on in this thesis. Other applications take more emphasis on the unusual and unique dispersion properties of Photonic Crystals, such as slow light propagation [15-17] or negative refraction (superlenses) $[19,20]$. In the following sections, we will give a short overview of the concept of photonic band structures, which provides the foundation of all those linear properties of Photonic Crystals.

\subsubsection{Basic Concepts from Solid-State Theory}

A $\mathrm{PhC}$ is characterized by a dielectric permittivity

$$
\epsilon(\vec{r}+\vec{R})=\epsilon(\vec{r})
$$

which is periodic with respect to the set $\mathcal{R}=\left\{\sum_{i=1}^{d} n_{i} \vec{a}_{i} ; n_{i} \in \mathbb{Z}\right\}$ of lattice vectors $\vec{R}$ that are generated by the primitive translations $\left\{\vec{a}_{i} ; i=1, \ldots, d\right\}$. The set $\mathcal{R}$ is referred to as the direct lattice [77].

The crystal breaks the continuous translation symmetry of homogeneous materials. Therefore, momentum conservation is no longer fulfilled within the crystal. Nevertheless, a discrete translation symmetry is retained, which can be identified with the conservation of the wave vector $\vec{k}$ up to any vector $\vec{G}$ that fulfills $\mathrm{e}^{\mathrm{i} \vec{G} \cdot \vec{R}}=1$. Thus we can define a reciprocal lattice as the set $\mathcal{G}=\left\{\sum_{i=1}^{d} n_{i} \vec{b}_{i} ; n_{i} \in \mathbb{Z}\right\}$ of reciprocal lattice vectors $\vec{G}$ generated by primitive translations $\vec{b}_{i}$ that fulfill $\vec{b}_{i} \cdot \vec{a}_{j}=2 \pi \delta_{i j}$. The reciprocal lattice is predetermined by the direct lattice (real-space lattice), and it belongs to the same point group. The Wigner-Seitz cell of the reciprocal lattice, i.e., the primitive unit cell that has the full symmetry of the lattice, is called first Brillouin zone. Any wave vector $\vec{k}$ can be folded back into the first Brillouin zone since it is only determined up to a reciprocal lattice vector. 


\section{Group Theory}

Besides the discrete translation symmetry with respect to an arbitrary lattice vector $\vec{R}$, the lattice of a PhC typically exhibits rotational and mirror symmetries. Formally, such symmetries are expressed by the space group of the lattice, which is defined by the set of symmetry operations (elements of the group), under which the object is invariant (is mapped onto itself). The point group of the lattice is a subgroup of the space group that excludes translations. Many features appearing in the band structure, such as avoided crossings or degeneracies, can be attributed to particular properties of the point group. Therefore, a careful group-theoretical analysis is of great importance in the study of fields within PhCs. This is especially the case for the generation of Wannier functions discussed in section 1.2.4.

We skip the detailed discussion about group theory here. A comprehensive description can be found, for instance, in [78, 79].

\section{Bloch theorem}

Solutions of the wave equation (1.9), or $(1.10),{ }^{5}$ within a $\mathrm{PhC}$ are eigensolutions of a PDE with periodic coefficients. They must obey the Bloch theorem, i.e., they must be lattice-periodic up to a phase factor $\mathrm{e}^{\mathrm{i} \overrightarrow{ } \vec{r}}$,

$$
\vec{H}_{\vec{k}}(\vec{r})=\mathrm{e}^{\mathrm{i} \vec{k} \vec{r}} \vec{u}_{\vec{k}}(\vec{r})
$$

where $\vec{u}_{\vec{k}}(\vec{r})$ is a lattice-periodic field. These so-called Bloch modes are labeled with the wave vector $\vec{k}$.

The Bloch theorem can be derived from group-theoretical considerations: The operator of a PDE with periodic coefficients and the translation operator $\hat{T}_{\vec{R}}$, which shifts a field by any lattice vector $\vec{R}$, commute. Therefore, the eigensolutions of the PDE are at the same time eigensolutions of the translation operator $\hat{T}_{\vec{R}}$. Since $\hat{T}_{\vec{R}}$ is unitary and commutes with any other translation operator $\hat{T}_{\vec{R}^{\prime}}$ (unitary Abelian group), it has only eigenvalues of the form $\mathrm{e}^{\mathrm{i} \phi_{\vec{R}}}$ (only one-dimensional unitary representations). $\phi_{\vec{R}}$ must be linear in $\vec{R}$ (since $\hat{T}_{\vec{R}} \hat{T}_{\vec{R}^{\prime}}=\hat{T}_{\vec{R}+\vec{R}^{\prime}}$ ), so we can write it in the form $\phi_{\vec{R}}=-\vec{k} \cdot \vec{R}$, which represents a definition for the wave vector $\vec{k}$ as a parameter. So we have

$$
\hat{T}_{\vec{R}} \vec{H}(\vec{r}) \equiv \vec{H}(\vec{r}-\vec{R})=\mathrm{e}^{-\mathrm{i} \vec{k} \vec{R}} \vec{H}(\vec{r}),
$$

which is equivalent to (1.15).

We can restrict the wave vector $\vec{k}$ to the first Brillouin zone, since $\vec{k}$ is equivalent to $\vec{k}+\vec{G}$. In this case, the periodic part $\vec{u}_{\vec{k}}(\vec{r})$ acquires an additional index $n$, which labels the solutions for all equivalent wave vectors $\vec{k}+\vec{G}, \vec{G} \in \mathcal{G}$, which are folded back into the first Brillouin zone and labeled with the same $\vec{k}$ now. Eq. (1.15) then reads

$$
\vec{H}_{n \vec{k}}(\vec{r})=\mathrm{e}^{\mathrm{i} \vec{k} \vec{r}} \vec{u}_{n \vec{k}}(\vec{r}) .
$$

\footnotetext{
${ }^{5}$ We will use the $\vec{H}$-field as an example in the following discussion. However, the same arguments can be applied to the $\vec{E}$-field formulation as well.
} 
Similarly, the $\vec{E}$-field solutions of (1.10) obey

$$
\vec{E}_{n \vec{k}}(\vec{r})=\mathrm{e}^{\mathrm{i} \vec{k} \vec{r}} \vec{u}_{n \vec{k}}(\vec{r}),
$$

and both (1.17) and (1.18) can be translated to scalar solutions of the 2D wave equations.

The Bloch theorem applies not only to the solutions of the wave equation in ideal $\mathrm{PhCs}$ but also to the guided modes of waveguides within PhCs. In such waveguides, the discrete translation symmetry is retained in the direction of the waveguide. Therefore, the wave vector of guided modes is always parallel to this direction.

\subsubsection{Band Structure and Band Gaps}

As alluded to above, scattering of light by the periodic distribution of the dielectric permittivity in a Photonic Crystal leads to the formation of a photonic band structure in the frequency spectrum of light. The linear dispersion relation (light cone) in vacuum or homogeneous materials is distorted by both the occurrence of Bragg resonances, which result from constructive interference of back-scattered waves from different lattice sites, and of Mie resonances, which are caused by the resonant scattering of the light at individual scatterers ("atoms") in a regime where the wavelength of light is of the order of the scatterer size. The interplay of these two scattering mechanisms gives rise to the complicated and feature-rich structure of the frequency spectrum of light in Photonic Crystals.

If we consider wave propagation into one direction, Bragg scattering always leads to the formation of frequency ranges, in which no propagating solutions are found. These so-called stop bands vary when the propagation direction is changed. If the stop bands for all possible directions have an overlap, this frequency range is called photonic band gap. If the band gap persists for all possible polarizations, we talk about a complete photonic band gap. The existence of (complete) band gaps depends on the strength of the Bragg and Mie resonances, which determine the extent of the individual stop bands. It also depends on the geometry and topology of the crystal lattice, which influences the relative position of the stop bands with respect to each other. In crystals with a more "circular" or "spherical" unit cell, wave propagation tends to be more isotropic, so that stop bands have a larger overlap. Therefore, such crystal geometries are more favorable for the formation of (complete) photonic band gaps.

As an example, Fig. 1.2 shows a band structure of a 2-dimensional $\mathrm{PhC}$, in which the two polarizations are plotted within the same diagram. While the band gaps for one polarization, such as the first band gap for $H$-polarization, can be very large, there is only a small complete band gap when considering both polarizations. This is also seen in Fig. 1.3, where the first band gap is plotted as a function of the radius of the air holes in the triangular lattice. It is difficult to get complete band gaps in $2 \mathrm{D}$ structures. The structure shown in Fig. 1.2 (triangular lattice of cylindrical air holes with radius $r=0.45 a$ in silicon) has such a complete band gap. Later on, we 

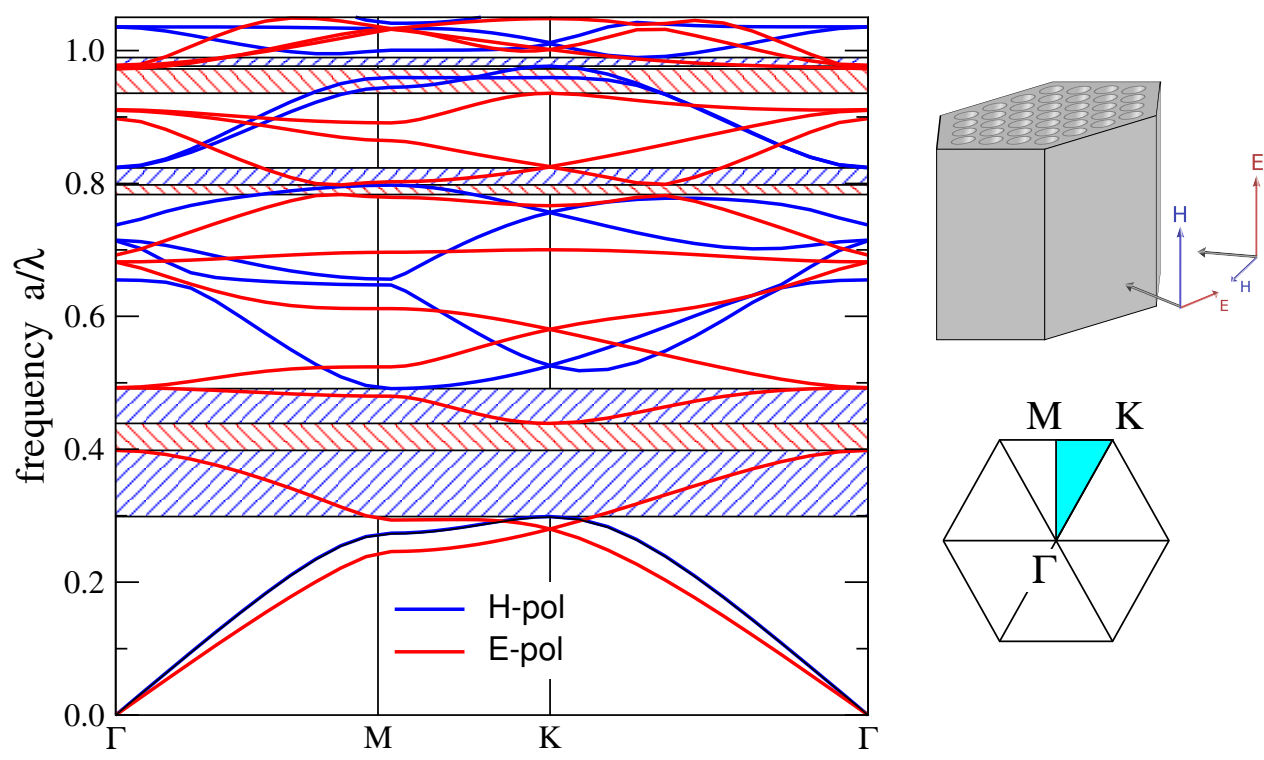

Figure 1.2.: Band structure of a 2D Photonic Crystal (triangular lattice of cylindrical air holes of radius $r=0.45 a$ in silicon $\left(\epsilon_{\mathrm{Si}}=12.0\right)$. Results for both polarizations ( $E$ - and $H$-polarization) are plotted into one diagram. The hatched areas depict the band gaps. For this structure, the first band gaps of the two polarizations overlap, so there exists a complete band gap in the vicinity of $a / \lambda=0.4$.

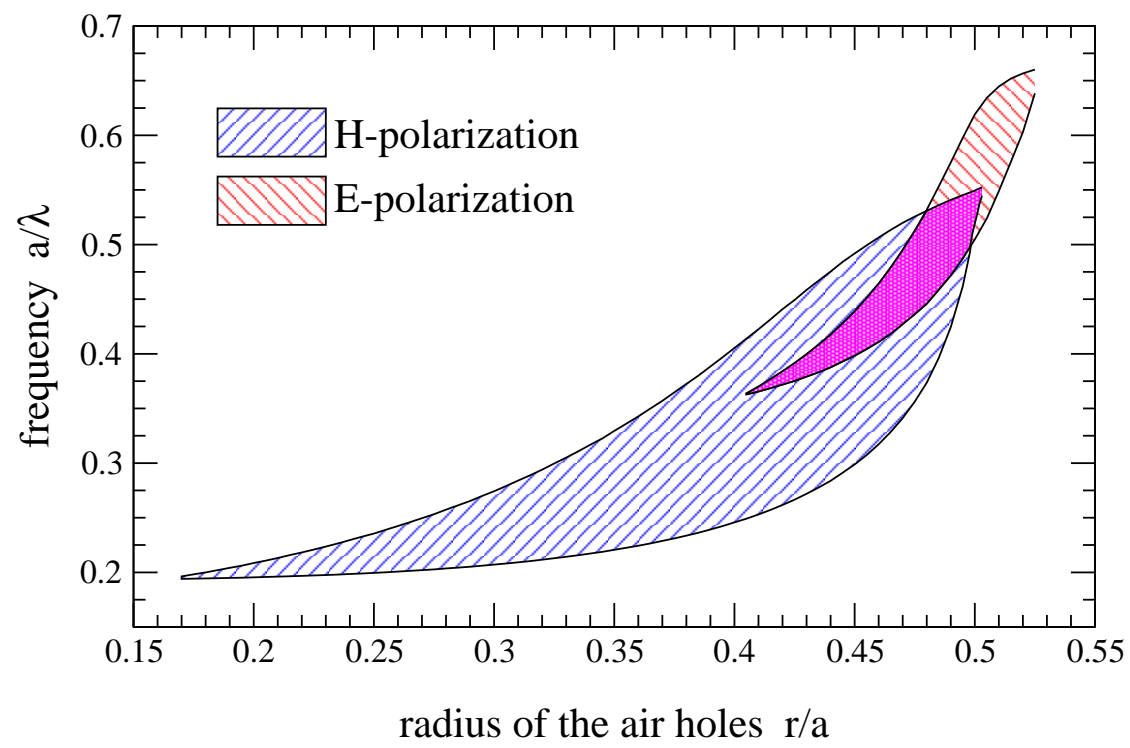

Figure 1.3.: Gap map for a triangular lattice of cylindrical air holes of different radii in silicon. A complete band gap (overlapping region of the band gaps for $E$ - and $H$-polarization) is only present for radii in the range $r=[0.405 a, 0.503 a]$. 
will concentrate on the large first band gap in $H$-polarization, in which single-mode waveguiding can be achieved.

\subsubsection{Bloch Modes and Wannier Functions}

First of all, to avoid unnecessary duplication of equations for $\vec{H}$ - and $\vec{E}$-field Bloch modes, which are otherwise equivalent, we would like to use Dirac notation where appropriate and define

$$
|n \vec{k}\rangle \equiv \begin{cases}\vec{H}_{n \vec{k}}(\vec{r}) & \text { in } \vec{H} \text {-field formulation, } \\ \vec{E}_{n \vec{k}}(\vec{r}) & \text { in } \vec{E} \text {-field formulation, }\end{cases}
$$

so that we can write scalar products more formulation-independent as

$$
\left\langle n \vec{k} \mid n^{\prime} \vec{k}^{\prime}\right\rangle \equiv\left\{\begin{array}{l}
\int_{\mathbb{R}^{d}} \mathrm{~d}^{d} r \vec{H}_{n \vec{k}}^{*}(\vec{r}) \vec{H}_{n^{\prime} \vec{k}^{\prime}}(\vec{r}), \\
\int_{\mathbb{R}^{d}} \mathrm{~d}^{d} r \vec{E}_{n \vec{k}}^{*}(\vec{r}) \epsilon(\vec{r}) \vec{E}_{n^{\prime} \vec{k}^{\prime}}(\vec{r}) .
\end{array}\right.
$$

We will return to the explicit notation when necessary.

The Bloch theorem, Eq. (1.17), forces that the time-harmonic solutions of the wave equation within a $\mathrm{PhC}$ (so-called Bloch modes) are plane waves $\mathrm{e}^{\mathrm{i} \vec{k} \vec{r}}$ modulated with an arbitrary lattice-periodic field $\vec{u}_{n \vec{k}}(\vec{r})$. There exist infinitely many Bloch modes for each wave vector $\vec{k}$ in the first Brillouin zone. Altogether, the set of Bloch modes for all wave vectors in the first Brillouin zone constitutes a complete and orthonormal basis, normalized with respect to a Wigner-Seitz cell of the $\mathrm{PhC}$,

$$
\left\langle n \vec{k} \mid n^{\prime} \vec{k}^{\prime}\right\rangle=\delta_{n n^{\prime}} \delta\left(\vec{k}-\vec{k}^{\prime}\right) .
$$

Each Bloch mode $|n \vec{k}\rangle$ is a representation of a symmetry group that is determined by the point group of the lattice, the symmetry group of the structure in the unit cell, and the symmetry group of the wave vector $\vec{k}$. For well-placed spherical or cylindrical "atoms" in the unit cell, having the maximum possible symmetry (continuous rotation symmetry), we can ignore the influence of the unit-cell structure. The wave vector typically has reduced symmetry and, therefore, the symmetry of an arbitrary Bloch mode is also reduced. At the $\Gamma$-point $(\vec{k}=0)$ or certain other high-symmetry points in the Brillouin zone (like the $K$-point in the 2D triangular lattice), the Bloch modes have the full lattice symmetry $\left(C_{6 v}\right.$ in the $2 \mathrm{D}$ triangular lattice), while along symmetry lines (e.g., $\overline{\Gamma K}$ ) or at high-symmetry points with reduced symmetry (e.g., the $M$-point in the 2D triangular lattice) they only retain the symmetry of a subgroup of the lattice point group. At arbitrary wave vectors in the Brillouin zone, the symmetry is completely lifted and the Bloch modes can be arbitrarily distributed.

The Bloch mode basis is well suited to describe fields within a slightly perturbed $\mathrm{PhC}$, since the basis is complete, knows about all symmetries of the lattice and the unit cell structure, and incorporates information about the dispersion relation and 
the band gaps of the PhC. Nevertheless, the Bloch modes are extended functions in space, so they are inefficient for describing localized fields such as defect states around local perturbations (defects) within the $\mathrm{PhC}$. Therefore, it is useful to introduce a basis equivalent to the Bloch mode basis but consisting of localized functions. This is the Wannier function basis.

Wannier functions $[80,81]$ are derived from the Bloch modes by a lattice Fourier transform,

$$
\vec{W}_{n \vec{R}}(\vec{r}) \equiv|n \vec{R}\rangle:=\frac{V_{\mathrm{WSC}}}{(2 \pi)^{3}} \int_{\mathrm{BZ}} \mathrm{d}^{3} k \mathrm{e}^{-\mathrm{i} \vec{k} \vec{R}}|n \vec{k}\rangle,
$$

where we integrate in wave-vector space over the first Brillouin zone of the reciprocal lattice. The Wannier functions are indexed by lattice vector $\vec{R}$, so each of them is associated to a specific site in the direct lattice, in contrast to a Bloch mode, which is assigned to a specific wave vector $\vec{k}$. By construction, Wannier functions at different lattice sites are related to each other by a simple shift in space,

$$
\vec{W}_{n \vec{R}}(\vec{r})=\vec{W}_{n \overrightarrow{0}}(\vec{r}-\vec{R}) .
$$

This means that it is only necessary to construct the Wannier functions $\vec{W}_{n \overrightarrow{0}}(\vec{r})$ associated to the origin site. The basis can then be easily completed by shifting these origin Wannier functions to every other lattice site $\vec{R}$.

The Wannier functions inherit many other properties of the Bloch modes they are constructed from. In particular, they form a complete and orthonormal basis,

$$
\left\langle n \vec{R} \mid n^{\prime} \vec{R}^{\prime}\right\rangle=\delta_{n n^{\prime}} \delta\left(\vec{R}-\vec{R}^{\prime}\right),
$$

which contains all information about symmetries and band gaps of the underlying $\mathrm{PhC}$. But in contrast to the Bloch modes, the Wannier functions can be generated such that they are localized (see section 1.2.4 below). This makes them ideally suited to describe localized defect states in a locally perturbed PhC. Fig. 1.4 gives a schematic illustration of the construction of Wannier functions from the Bloch modes and shows the first nine Wannier functions for the example system in Fig. 1.2. It also suggests that the generation of Wannier functions is a highly nontrivial task.

\subsubsection{Wannier Function Generation}

Despite the clear formal definition of Wannier functions, Eq. (1.22), computing them directly via (1.22) leads to functions with poor localization properties and erratic behavior. This has basically two reasons.

1. Indeterminacy of Bloch modes: The Bloch functions $\vec{H}_{n \vec{k}}(\vec{r})$ are determined except for a global phase factor,

$$
\vec{H}_{n \vec{k}}(\vec{r}) \longleftrightarrow \vec{H}_{n \vec{k}}(\vec{r}) \mathrm{e}^{\mathrm{i} \phi_{n \vec{k}}},
$$

where $\phi_{n \vec{k}}$ can be chosen for each Bloch mode individually. Numerically computed Bloch modes may therefore show a random relationship of the individual phases $\phi_{n \vec{k}}$. 


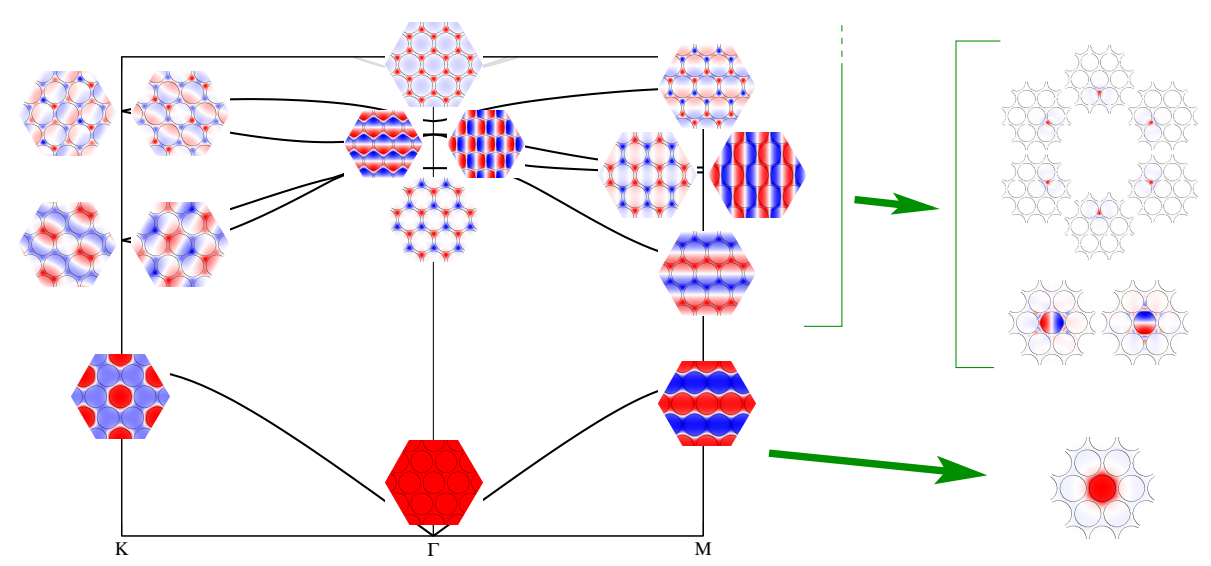

Figure 1.4.: Schematic illustration of the construction of Wannier functions from Bloch modes. The example shows the $2 \mathrm{D}$ band structure and Bloch modes $\left(H_{z}\right.$ component) corresponding to Fig. 1.2. While the first band can be treated separately and reveals a monopole-like Wannier function, it is apparent that the upper bands are significantly entangled and have to be treated simultaneously. Two dipole-like Wannier functions and a star of six hybridized Wannier functions can be constructed when taking bands two to nine into account. [82].

As a consequence, the Wannier functions resulting from (1.22) are not unique and may be largely extended and asymmetric.

2. Entanglement of bands: The band index $n$ for entangled bands is a poor criterion for extracting single bands, since it orders the Bloch modes by their frequency only and completely neglects symmetries and mode structure. When band crossings occur, the band index is not constant along a band of certain symmetry and mode structure. Bloch modes with the same band index may have totally different symmetries and mode structure. Consequently, Wannier functions constructed from such Bloch modes with the same band index typically have poor symmetry properties.

These two complications make it inevitable to generalize Eq. 1.22 to account for both the adjustment of global phase factors of Bloch modes and the disentanglement of bands. We therefore allow the Bloch modes at each wave vector $\vec{k}$ to be mixed by a unitary transformation $U_{m n}^{(\vec{k})}$,

$$
|n \vec{k}\rangle \rightsquigarrow \sum_{m} U_{m n}^{(\vec{k})}|m \vec{k}\rangle .
$$

This transformation includes the global phase adjustment of a single band, in which case the sum has only one summand $m=n$, and the unitary transformation reduces to a phase factor $\mathrm{e}^{\mathrm{i} \phi} \phi_{n \vec{k}}$ like in (1.25). We call the new basis functions in (1.26) generalized Bloch modes since, in general, these functions are no longer eigensolutions of the wave equation for a certain frequency. Finally, by using generalized Bloch modes, (1.26), as input for the generation of Wannier functions, (1.22), we get enough free parameters to be able to construct highly localized and symmetric Wannier 
functions.

Nevertheless, the difficulty is now only shifted to the determination of appropriate unitary transformations $U_{m n}^{(\vec{k})}$ that make the Wannier functions fit some predefined criteria best. Criteria may be maximal localization and/or symmetry of the Wannier functions. The localization, for instance, can be quantified by the spread functional,

$$
\Omega=\sum_{m}\left[\left\langle m \overrightarrow{0}\left|\vec{r}^{2}\right| m \overrightarrow{0}\right\rangle-(\langle m \overrightarrow{0}|\vec{r}| m \overrightarrow{0}\rangle)^{2}\right]=\min .
$$

Furthermore, the grouping of bands, reflected by the range of the summation index $m$ in (1.26) and (1.27) has to be carefully chosen.

For further details, we would like to refer the reader to the dissertation of Matthias Schillinger [82], in which he outlines and extends the instructions for Wannier function generation by Marzari, Vanderbuilt and Souza [83, 84], originally developed in the context of electronic band structure theory.

\subsubsection{Single-Pore Infiltration}

The diverse fabricational techniques to realize $\mathrm{PhCs}$ and $\mathrm{PhC}$ defect structures [2641] can not be covered in detail here. Instead of reviewing this subject, we shortly discuss only one technique, namely the single-pore infiltration of 2D PhCs [50], which we will use as a basis for the circuit design in chapter 5 .

In macroporous silicon 2D PhCs, such as model system 2 used in chapters 4 and 5, defects can be created by infiltrating individual pores with materials of different dielectric permittivity, for instance, low-index materials such as polymers. Point defects can be created very flexibly inside an existing $2 \mathrm{D} \mathrm{PhC}$ with this technique. It allows to separate the creation of the ideal $\mathrm{PhC}$ and the writing of customized defect structures inside the crystal into a two-step process. It may even be possible to rewrite circuits inside the $\mathrm{PhC}$ by using appropriate materials that can be removed. First successful experiments with single-pore infiltration have been reported in [85].

Furthermore, the infiltration of tunable materials such as nematic liquid crystals (LCs) [55] allows us to incorporate actively tunable functional elements $[57,58,76,86]$ into PhC circuits. LCs can be tuned by applying an external electric field, which aligns the anisotropic LC molecules such that their (nematic) director (the averaged orientation of the molecules) points along the field. The LC is characterized by an ordinary and an extraordinary component of the dielectric tensor, $\epsilon_{o}$ and $\epsilon_{e}$, respectively. In addition, the director angle $\theta$ within the plane of propagation in the $2 \mathrm{D} \mathrm{PhC}$ fixes the orientation of the LC. Those parameters are illustrated in Fig. 1.5. More details can be found in the diploma thesis of Patrick Mack [76]. The complete alignment of the LC molecules along the external electric field is a simplification compared to the experimental situation, where the molecules are, for instance, largely influenced by surface forces [45]. Nevertheless, we are confident that this concept is experimentally realizable.

Another option is to infiltrate single pores with magneto-optical materials to obtain non-reciprocal functionality, such as an optical isolator or circulator [87], within $\mathrm{PhC}$ 

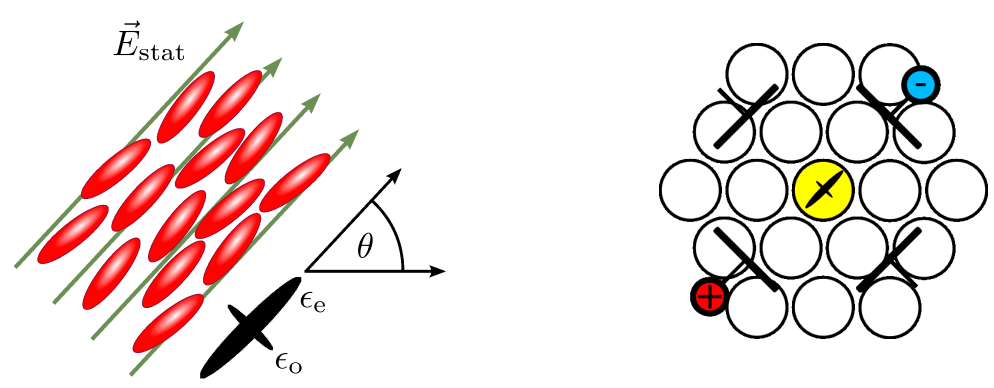

Figure 1.5.: Liquid crystals and their tunability by an electric field. The three parameters $\epsilon_{\mathrm{o}}, \epsilon_{\mathrm{e}}$ and $\theta$ fully characterize the LC in this simplified picture. In reality, surface forces may strongly influence the alignment. Pictures by courtesy of Patrick Mack [76].

circuits.

The single-pore infiltration has the potential to become a flexible and powerful alternative to well established technologies, such as focussed ion beam techniques. The point defects within model system 2 discussed later on will all be based on single-pore infiltration. Although the defect pore permittivity is the only remaining degree of freedom for creating defects in the $\mathrm{PhC}$, i.e., no radius or shape variations or hole displacements are allowed, there still is enough flexibility to design large-scale $\mathrm{PhC}$ circuits, as will be demonstrated in chapter 5 .

\subsection{Conventional Circuits}

In view of chapter 5, where directional couplers and Mach-Zehnder interferometers in $\mathrm{PhCs}$ are investigated, we briefly introduce in the present section some general concepts and working principles concerning those two device classes.

\subsubsection{Directional Coupler}

Directional couplers (DCs) were introduced as passive components for microwave circuits and have a long history, reaching back to the year 1922 [88]. Since then, a huge number of variants have been designed, and the concept has been extended to optical components; however, the basic working principles remained the same.

A DC consists of two waveguides that are coupled over a certain distance. To be specific, we first consider a typical fiber-optical DC schematically depicted in Fig. 1.6. It consists of two fibers approaching each other so that light can couple between them. We assume that the fibers are identical (symmetric DC) and single-mode, and we ignore reflections. Light enters the DC through the waveguide ports 1 or 2 . In the coupling section, the field is a superposition of the two fundamental modes of the dual-mode waveguide formed by the two coupled fibers. Those modes are even and odd with respect to a mirror reflection on the plane parallel to and equidistant from both fibers. They propagate with different wave vectors $k_{\text {even }}$ and $k_{\text {odd }}$, respectively. 


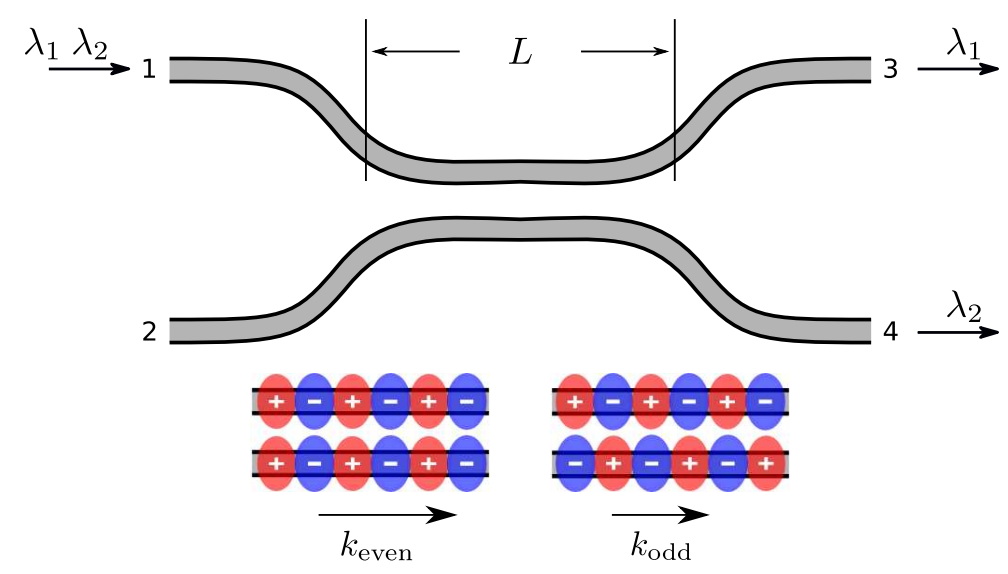

Figure 1.6.: Typical fiber-optical DC consisting of two fibers approaching each other, so that light can couple between them over a certain distance. Light entering in ports 1 and 2 (on the left) exits in ports 3 and 4 (on the right), assuming that no reflections occur. The ratio of the output amplitudes in ports 3 and 4 depends on the length of the coupling section and the wavelength of the light. The insets depict the fundamental modes in the coupling section, an even and an odd mode, composed of the original modes in the single fibers.

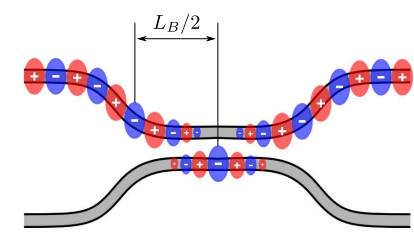

(a) bar state

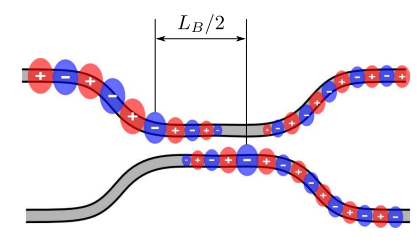

(b) $3 \mathrm{~dB}$ coupling

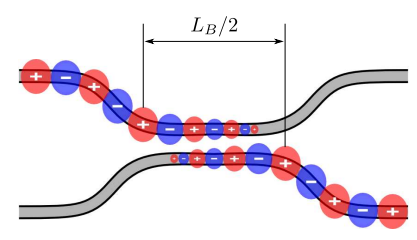

(c) cross state

Figure 1.7.: Modes of operation of a typical fiber-optical DC. Light launched into one waveguide (a) exits in the same waveguide, (b) is split up equally into both waveguides, or (c) is coupled over to the other waveguide. In this particular illustration, the wavelength of the light is varied instead of the length of the coupler (wider/narrower ellipses).

Due to mode beating, the resulting field shifts periodically between the two fibers with a period of $L_{B}=2 \pi /\left|k_{\text {even }}-k_{\text {odd }}\right|$, called beat length. At the end of the coupling section, the resulting field pattern determines how much light exits the DC in the waveguide ports 3 and 4 .

Thus, if the coupler length $L$ is equal to $L_{B}$ (or a multiple of it), light launched into one of the waveguides exits in the same waveguide (bar state), while if the coupler length is half the beat length (or an odd multiple of it), the light is completely coupled over to the other waveguide (cross state). In between (i.e. for $L=1 / 4 L_{B}, 3 / 4 L_{B}, \ldots$ ), the coupler splits up the light equally into both waveguides ( $3 d B$ coupling). These modes of operation are illustrated in Fig. 1.7. 


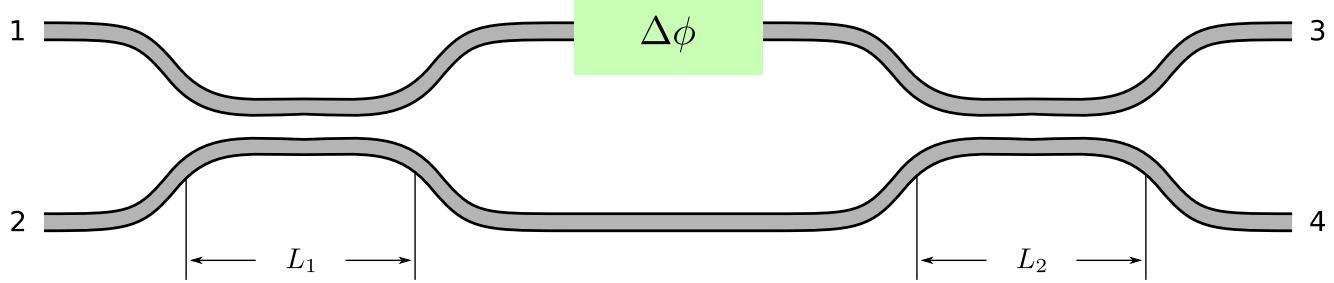

Figure 1.8.: Four-port MZI composed of two DCs and a phase shifting component in the upper arm. By manipulating the relative phase shift $\Delta \phi$ in the upper arm, signals input in port 1 and 2 can be flexibly routed to ports 3 and 4 .

\subsubsection{Mach-Zehnder interferometer}

Interferometers represent an important building block for modern optical circuitry. The underlying principle is the manipulation of the intensity of an output signal by applying controlled phase shifts to the signal within the device. The type of interferometer proposed by Zehnder [89] and Mach [90] and named after them consists of two separate arms, in which the phase of the signal can be manipulated separately. The simplest realization of a Mach-Zehnder interferometer (MZI) is composed of a beam splitter and a beam combiner, which are connected over two separate waveguides. In such a setup, a beam is split into two equal beams that are in phase. After the controlled phase shifts within the interferometer arms, the beams are combined and interfere with each other. Depending on the relative phase, the interference is constructive or destructive, resulting in an amplitude modulation of the output beam.

A slightly more complicated Mach-Zehnder interferometer is shown in Fig. 1.8. This version has two input ports 1 and 2, which can be independently operated. They are coupled over a certain distance (see the previous section about the directional coupler, DC) before the signal is split again into the two separate MZI arms. The combination of the arms is again accomplished by a DC, so that there are two output ports 3 and 4 . The simple MZI with only one input port and an equal partitioning into the two arms can, for instance, be reproduced by a single input in port 1 and an operation wavelength, for which the DC at the input side splits up the signal equally (3dB coupling) and the DC at the output side combines two phase-matched signals into one output port. Besides this special case, such a more general MZI can be operated in many different ways.

As an example, we present the operation of the MZI in Fig. 1.8 as an optical switch, which routes a signal from input port 1 to either output port 3 or 4 . The two states of the switch, illustrated in Fig. 1.9, correspond to different relative phase shifts $\Delta \phi=0$ and $\pi$, respectively. In chapter 5 , we will discuss exactly this case for an MZI based on a $\mathrm{PhC}$ with phase shifts induced by tuning the director angle of nematic liquid crystals infiltrated in the $\mathrm{PhC}$. 


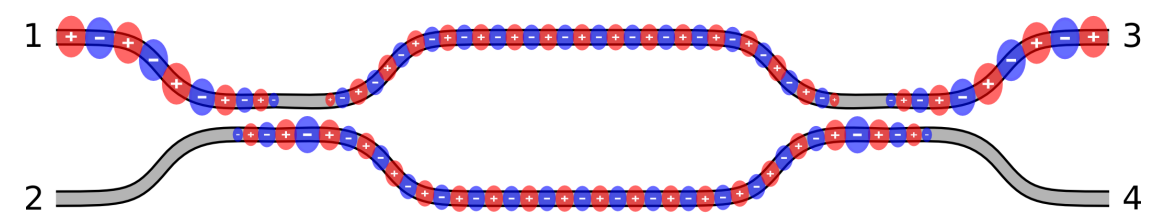

(a) $\Delta \phi=0$

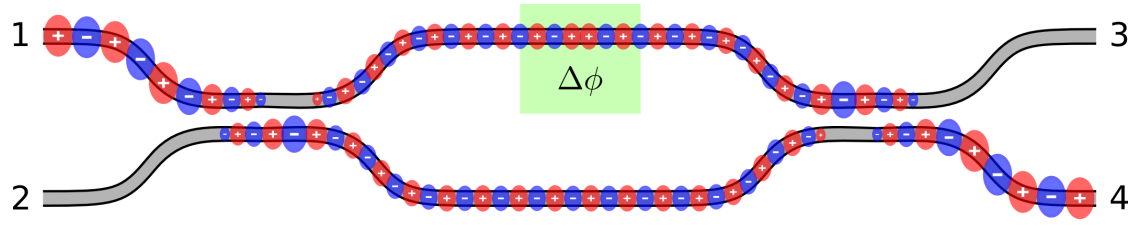

(b) $\Delta \phi=\pi$

Figure 1.9.: Two states of a switch based on an MZI. A signal input in port 1 is routed either into port 3 or port 4 , depending on the relative phase shift $\Delta \phi$ between the two arms. 
1. Fundamentals 


\section{Wannier Function Expansion}

In this chapter, we introduce the Wannier function expansion as a method to discretize the wave equation for defect structures in Photonic Crystals. First, we conceptually introduce defect structures as additional contributions to the periodic dielectric constant of a Photonic Crystal. Then, we expand the fields into Wannier functions, insert the expansion into the wave equation, and get a finite matrix problem by truncating the Wannier function basis both in real space and in band index. Details about the overlap matrices involved in this process are given. The overlap matrices can be calculated once for a given Photonic Crystal and for different point defect prototypes, and can be reused afterwards for any constellation of point defects. This allows a rapid design of functional elements in a given Photonic Crystal, once the Wannier functions and corresponding overlap matrices are generated. Finally, we give precise instructions on how to calculate cavity modes, waveguide modes and scattering matrices of functional elements and discuss the optimization of functional elements.

\subsection{Defect Structures in Photonic Crystals}

Defect Structures embedded in PhCs, as they are illustrated in Fig. 2.1, can be conceptually described by an additional contribution to the periodic dielectric constant of a $\mathrm{PhC}$,

$$
\epsilon(\vec{r})=\epsilon_{\mathrm{p}}(\vec{r})+\delta \epsilon(\vec{r}) .
$$

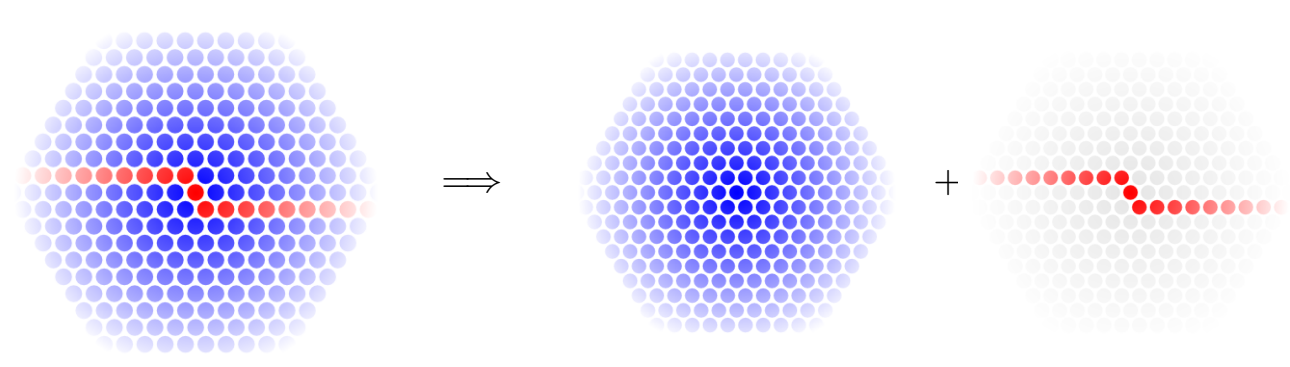

Figure 2.1.: Illustration of defect structures embedded in a PhC. The structure can be described by an additional contribution to the periodic dielectric permittivity of the underlying $\mathrm{PhC}$. This contribution itself can typically be split into contributions of point defects that are located at different lattice sites. 
Alternatively, we can use the inverse periodic dielectric constant $\eta_{\mathrm{p}}(\vec{r}):=\epsilon_{\mathrm{p}}^{-1}(\vec{r})$ as a reference and write

$$
\eta(\vec{r}):=\epsilon^{-1}(\vec{r})=\eta_{p}(\vec{r})+\delta \eta(\vec{r}),
$$

so that

$$
\delta \eta(\vec{r})=\frac{-\delta \epsilon(\vec{r})}{\epsilon_{\mathrm{p}}(\vec{r})\left[\epsilon_{\mathrm{p}}(\vec{r})+\delta \epsilon(\vec{r})\right]} .
$$

The defect part $\delta \epsilon(\vec{r})$ (or $\delta \eta(\vec{r})$, respectively ${ }^{1}$ ) often has a complicated structure. However, in most cases, it can be further simplified by decomposing it into a sum of point defect contributions.

\subsubsection{Point Defects}

We define point defects as zero-dimensional defects that are restricted to one unit cell of the Photonic Crystal. ${ }^{2}$ A point defect at the origin lattice site can be described by

$$
\delta \epsilon(\vec{r})=\Delta \epsilon \cdot \Theta(\vec{r}),
$$

where $\Theta(\vec{r})$ is a form factor describing the shape of the defect and $\Delta \epsilon$ is the defect strength. For simple point defects, such as filled or shifted pores in macroporous silicon, missing rods or rods with different radius in rod systems, $\Theta(\vec{r})$ is piecewise constant,

$$
\Theta_{\text {simple }}(\vec{r})= \begin{cases} \pm 1 & \text { for } \vec{r} \text { in regions with changed dielectric constant } \\ 0 & \text { for } \vec{r} \text { elsewhere. }\end{cases}
$$

For more complex point defects, $\Theta(\vec{r})$ may be a continuous function in the interval $[-1,1]$.

In this work we are mainly considering two-component Photonic Crystals that consist of a background matrix with dielectric constant $\epsilon_{b}$, in which pores (or rods) with dielectric constant $\epsilon_{a}$ are arranged periodically. Point defects are created by modifying one pore (or rod, respectively) in the Photonic Crystal. On the one hand, we can change the shape of the pore, increase or decrease the radius or dislocate the pore within the unit cell. In these cases, the defect strength is given by $\Delta \epsilon=\epsilon_{b}-\epsilon_{a}$. On the other hand, we can incorporate other materials into the two-component $\mathrm{PhC}$, e.g. by infiltrating a single pore and thereby changing the dielectric constant inside it. In this case, the defect strength is given by $\Delta \epsilon=\epsilon_{\mathrm{def}}-\epsilon_{a}$, where $\epsilon_{\mathrm{def}}$ is the dielectric constant of the infiltrated material. In general, the freedoms of creating simple point defects can be combined arbitrarily to form complex point defects.

\footnotetext{
${ }^{1}$ Since the treatment of $\delta \eta(\vec{r})$ is perfectly analogous to $\delta \epsilon(\vec{r})$, we restrict ourselves to the discussion of $\delta \epsilon(\vec{r})$ in most cases but use the developed expressions in both contexts later on.

${ }^{2}$ We could define point defects in a more general sense, also allowing them to exceed one unit cell. We use the restriction to one unit cell only for the sake of simplicity when defining overlap matrices below.
} 


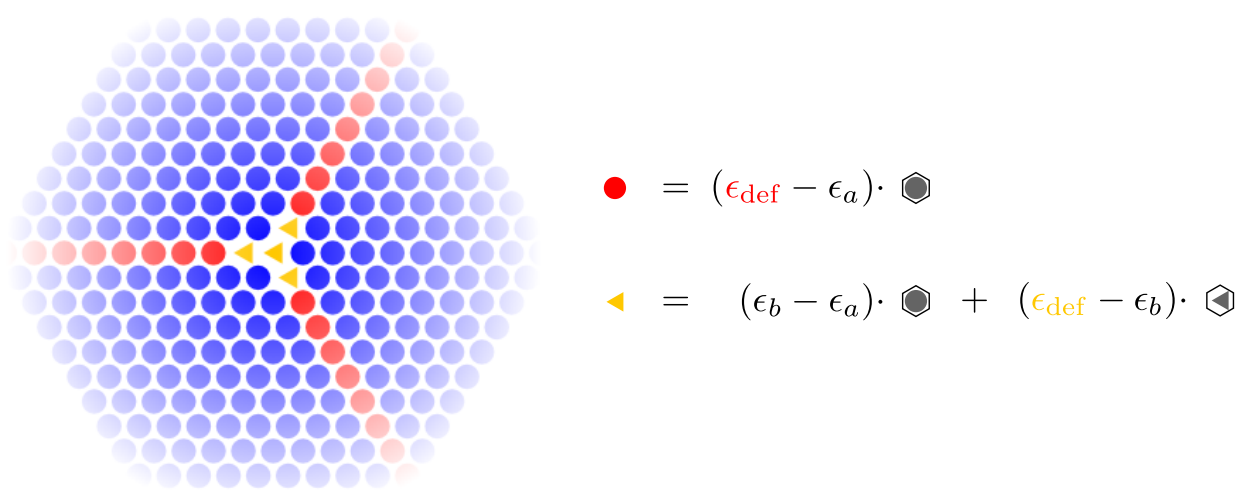

Figure 2.2.: Defect structure split up into point defects, whose dielectric permittivity can be expressed as a product of defect strength times form factor. The triangle defect is an example for a point defect that is composed of two form factors.

By combining several point defects at different positions $\vec{R}_{m}$,

$$
\delta \epsilon(\vec{r})=\sum_{m} \delta \epsilon_{m}\left(\vec{r}-\vec{R}_{m}\right) \quad \text { with } \quad \delta \epsilon_{m}(\vec{r})=\Delta \epsilon_{m} \Theta_{m}(\vec{r}),
$$

complex defect structures in a $\mathrm{PhC}$ can be easily composed. In a typical situation, only a few different prototypes of point defects $\delta \epsilon_{m}(\vec{r})$ are used and the complex structures are built by just copying a prototype and moving the copy to the proper lattice site $\vec{R}_{m}$.

\subsection{Wave Equations in Wannier Basis}

As explained in section 1.2.3, Wannier functions represent an orthonormal basis of localized functions, which is adapted to the specific $\mathrm{PhC}$ host material. This basis is ideally suited to describe localized fields in the perturbed $\mathrm{PhC}$. Each Wannier function $W_{n \vec{R}}(\vec{r})$ is labeled by a band index $n$ and the lattice vector $\vec{R}$ of the lattice site where it is centered. The band index does not necessarily correspond to a single band in the band structure, due to the entanglement of bands, which is resolved in the generation of the Wannier functions. If we take an infinite number of bands and lattice vectors into account, the Wannier basis is complete. By suitably truncating the number of bands and the spacial region, we obtain a finite basis that covers the localized fields well within the extent of the $\mathrm{PhC}$ defect structure. To simplify the notation, we introduce a composite index $\alpha=\{n, \vec{R}\}$, denoted by Greek letters, so that $W_{\alpha}(\vec{r}) \equiv W_{n \vec{R}}(\vec{r})$. Any overlap matrix element connecting two specific Wannier functions $W_{n \vec{R}}(\vec{r})$ and $W_{n^{\prime} \vec{R}^{\prime}}(\vec{r})$ can then be written in the form $M_{\alpha \beta} \equiv M_{\vec{R} \vec{R}^{\prime}}^{n n^{\prime}}$, with only two indices. 


\subsubsection{D H-polarization}

We start with the wave equation (1.11) for $H$-polarized fields in 2D systems,

$$
\left[\nabla_{2} \cdot\left\{\eta_{\mathrm{p}}(\vec{r})+\delta \eta(\vec{r})\right\} \nabla_{2}+\frac{\omega^{2}}{c^{2}}\right] H(\vec{r})=0,
$$

where the contributions to $\eta(\vec{r})$ of the periodic crystal and the defect structure have been separated and emphasized by different colors. We expand the field $H(\vec{r})$ into Wannier functions,

$$
H(\vec{r})=\sum_{\beta} H_{\beta} W_{\beta}(\vec{r})
$$

insert this expansion into (2.7), and project onto an arbitrary Wannier function $W_{\alpha}^{*}(\vec{r})$ to get

$$
\sum_{\beta}\left[C_{\alpha \beta}+D_{\alpha \beta}-\Lambda A_{\alpha \beta}\right] H_{\beta}=0 \quad \forall \alpha
$$

with

$$
\begin{aligned}
A_{\alpha \beta} & =\int \mathrm{d}^{2} r W_{\alpha}^{*}(\vec{r}) W_{\beta}(\vec{r}) \\
C_{\alpha \beta} & =\int \mathrm{d}^{2} r\left(\nabla_{2} W_{\alpha}^{*}(\vec{r})\right) \eta_{\mathrm{p}}(\vec{r})\left(\nabla_{2} W_{\beta}(\vec{r})\right) \\
D_{\alpha \beta} & =\int \mathrm{d}^{2} r\left(\nabla_{2} W_{\alpha}^{*}(\vec{r})\right) \delta \eta(\vec{r})\left(\nabla_{2} W_{\beta}(\vec{r})\right)
\end{aligned}
$$

and

$$
\Lambda=\frac{\omega^{2}}{c^{2}}
$$

In the expressions for $C_{\alpha \beta}$ and $D_{\alpha \beta}$, the gradient operator has been shifted to the Wannier function on the left,

$$
\int \mathrm{d}^{2} r W_{\alpha}^{*}(\vec{r}) \nabla_{2} \cdot \eta(\vec{r}) \nabla_{2} W_{\beta}(\vec{r})=-\int \mathrm{d}^{2} r\left(\nabla_{2} W_{\alpha}^{*}(\vec{r})\right) \eta(\vec{r})\left(\nabla_{2} W_{\beta}(\vec{r})\right),
$$

resulting in the minus sign in (2.9). Note that the matrix $A_{\alpha \beta}$, Eq. (2.10), becomes an identity matrix, $A_{\alpha \beta}=\delta_{\alpha \beta}$, if the Wannier functions are orthonormalized with respect to the $H$-field scalar product, (1.20). Since the orthonormality condition is not always fulfilled, for instance when extending the Wannier function basis by some auxiliary functions that may improve convergence (cf. chapter 4), we take into account the numerically calculated matrix $A_{\alpha \beta}$ instead of assuming it to be an identity matrix. Thus we are able to easily use non-orthonormal bases without adjustments to the algorithms presented later on. 


\subsubsection{D E-polarization}

The wave equation (1.12) for $E$-polarized fields in 2D systems,

$$
\left[\Delta_{2}+\frac{\omega^{2}}{c^{2}}\left\{\epsilon_{\mathrm{p}}(\vec{r})+\delta \epsilon(\vec{r})\right\}\right] E(\vec{r})=0
$$

is treated similarly. The expansion into Wannier functions,

$$
E(\vec{r})=\sum_{\beta} E_{\beta} W_{\beta}(\vec{r})
$$

inserted into (2.15), leads to an algebraic equation formally identical to (2.9),

$$
\sum_{\beta}\left[C_{\alpha \beta}+D_{\alpha \beta}-\Lambda A_{\alpha \beta}\right] E_{\beta}=0 \quad \forall \alpha
$$

albeit with different definitions of the overlap matrices and the frequency term,

$$
\begin{aligned}
A_{\alpha \beta} & =-\int \mathrm{d}^{2} r W_{\alpha}^{*}(\vec{r}) \Delta_{2} W_{\beta}(\vec{r}) \\
C_{\alpha \beta} & =\int \mathrm{d}^{2} r W_{\alpha}^{*}(\vec{r}) \epsilon_{\mathrm{p}}(\vec{r}) W_{\beta}(\vec{r}) \\
D_{\alpha \beta} & =\int \mathrm{d}^{2} r W_{\alpha}^{*}(\vec{r}) \delta \epsilon(\vec{r}) W_{\beta}(\vec{r})
\end{aligned}
$$

and

$$
\Lambda=\frac{c^{2}}{\omega^{2}}
$$

Note that in this case, the matrix $C_{\alpha \beta}$ (instead of $A_{\alpha \beta}$ in the $H$-polarized case) becomes an identity matrix, $C_{\alpha \beta}=\delta_{\alpha \beta}$, if the Wannier function basis is orthonormalized.

\subsubsection{D wave equations}

It is straightforward to show that the $3 \mathrm{D}$ wave equations (1.7) and (1.8) can be cast into the same formal matrix equation (2.9) (or (2.17)), but with different definitions of the overlap matrices and the frequency term. In 3D, fields and Wannier functions become vector-valued, but the Wannier function basis is still indexed by band index $n$ and lattice site $\vec{R}$, and the overlap matrix elements are again scalar quantities. Therefore, the numerical treatment of the $3 \mathrm{D}$ wave equations formulated in terms of overlap matrices is the same as in $2 \mathrm{D}$. Since we will only consider the $2 \mathrm{D}$ case later on, we skip the detailed definitions of the overlap matrix elements in 3D here. 


\subsubsection{Splitting into Point Defect Contributions}

If the defect contribution $\delta \epsilon(\vec{r})$ to the dielectric permittivity (or $\delta \eta(\vec{r})$, respectively) can be decomposed into point defects according to (2.6), the same decomposition can be applied to the defect overlap matrix $D_{\alpha \beta}$,

$$
D_{\alpha \beta}=\sum_{m} \Delta \epsilon_{m} B_{\alpha-\vec{R}_{m}, \beta-\vec{R}_{m}}^{(m)}
$$

with

$$
B_{\alpha \beta}^{(m)}=\int \mathrm{d}^{2} r W_{\alpha}^{*}(\vec{r}) \Theta_{m}(\vec{r}) W_{\beta}(\vec{r}) .
$$

The overlap matrix $B_{\alpha \beta}^{(m)}$ only depends on the form factor $\Theta_{m}(\vec{r})$ of the $m$-th point defect, independent of its location. The displacement $\vec{R}_{m}$ of the $m$-th point defect is accounted for in (2.22) by the shift of the indices $\alpha \rightarrow \alpha-\vec{R}_{m}$ and $\beta \rightarrow \beta-\vec{R}_{m}$. This is possible because of the special translation property (1.23) of the Wannier functions, so that

$$
\int \mathrm{d}^{2} r W_{\alpha}^{*}(\vec{r}) \Theta_{m}\left(\vec{r}-\vec{R}_{m}\right) W_{\beta}(\vec{r})=\int \mathrm{d}^{2} r W_{\alpha-\vec{R}_{m}}^{*}(\vec{r}) \Theta_{m}(\vec{r}) W_{\beta-\vec{R}_{m}}(\vec{r})
$$

or, in other words, the relative shift of the form factor can be cast into a shift in the indices of the Wannier functions involved in the overlap integral and, consequently, into a shift of the indices of the overlap matrix $B_{\alpha \beta}^{(m)}$ itself.

The splitting of the defect overlap matrix $D_{\alpha \beta}$ into contributions of point defects allows to assemble many different defect configurations within a $\mathrm{PhC}$ from only a few form-factor overlap matrices $B_{\alpha \beta}^{(m)}$. In simple cases where all point defects have the same form factor, e.g., in the case of holes filled with different materials in macroporous silicon, only one form-factor overlap matrix $B_{\alpha \beta}$ is necessary. The dielectric permittivity difference of the filling materials is cast into the defect strength $\Delta \epsilon_{m}$. The matrices $B_{\alpha \beta}^{(m)}$ can be precomputed and stored on disk once like the other overlap matrices $A_{\alpha \beta}$ and $C_{\alpha \beta}$.

\subsubsection{Properties of Overlap Matrices}

The overlap matrices have some useful properties, which we want to point out separately. First of all, they all are constructed from the Wannier function basis, which has the special translation property $W_{\alpha+\vec{R}}(\vec{r})=W_{\alpha}(\vec{r}-\vec{R})$, Eq. (1.23). Since $A_{\alpha \beta}$ exclusively involves Wannier functions, and $C_{\alpha \beta}$ additionally contains only the periodic contribution to the dielectric permittivity, $\epsilon_{\mathrm{p}}(\vec{r})$, these two overlap matrices are invariant under a simultaneous translation of their indices $\alpha$ and $\beta$ by an arbitrary lattice vector $\vec{R}$,

$$
A_{\alpha-\vec{R}, \beta-\vec{R}}=A_{\alpha \beta} \quad C_{\alpha-\vec{R}, \beta-\vec{R}}=C_{\alpha \beta} .
$$

On the other hand, the defect overlap matrices $B_{\alpha \beta}^{(m)}$, from which the matrix $D_{\alpha \beta}$ is constructed, do not have this index translation symmetry, since the form factor 


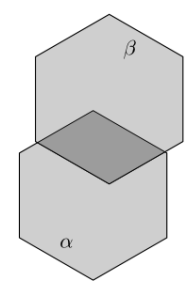

(a) $A_{\alpha \beta}$

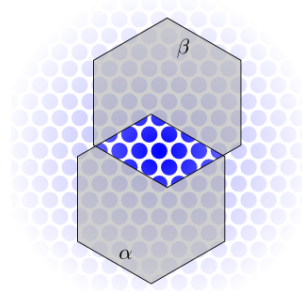

(b) $C_{\alpha \beta}$

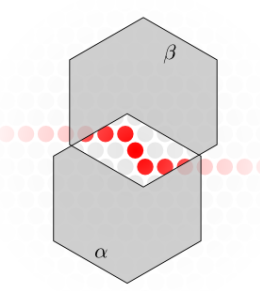

(c) $D_{\alpha \beta}$

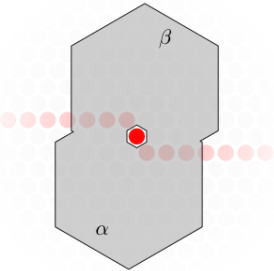

(d) $B_{\alpha \beta}^{(m)}$

Figure 2.3.: Integration domains for overlap matrices. The localized Wannier functions $|\alpha\rangle$ and $|\beta\rangle$ (and their derivatives) have a finite support, symbolized by gray hexagonal domains. The overlapping region is the integration domain for the matrices $A_{\alpha \beta}, C_{\alpha \beta}$ and $D_{\alpha \beta}$, which involve different parts in the wave equation. The defect overlap matrix $D_{\alpha \beta}$ can be further decomposed into matrices $B_{\alpha \beta}^{(m)}$, for which the integration is only performed over the form factor of the $m$-th point defect.

$\Theta_{m}(\vec{r})$ is fixed at the origin lattice site. The index translation symmetry can be used to minimize the number of overlap matrix elements that need to be computed and stored.

Secondly, due to the rapid decay of Wannier functions (and their derivatives) with distance to their central lattice site, overlap integrals quickly become very small when the distance $\left|\vec{R}_{\alpha}-\vec{R}_{\beta}\right|$ between the centers of two Wannier functions $W_{\alpha}(\vec{r})$ and $W_{\beta}(\vec{r})$ increases. Therefore, we introduce a numerical parameter $R_{\max } \in \mathbb{N}$ that measures the maximum distance of two Wannier function centers, above which any overlap integral is neglected,

$$
M_{\alpha \beta} \equiv 0 \quad \forall \alpha, \beta \text { with }\left|\vec{R}_{\alpha}-\vec{R}_{\beta}\right|>R_{\max } a .
$$

Typically, it is safe to neglect overlap integrals of Wannier functions whose central lattice sites are separated by more than $R_{\max }=4$ lattice constants. In many cases, it is even sufficient to use $R_{\max }=3$. This largely depends on the localization properties of the Wannier functions though. Note that the choice of $R_{\max }=1$, which would correspond to a tight-binding approximation with only nearest-neighbor interaction in electronic structure calculations, generally does not give satisfying results.

\subsection{Classes of Defect Structures}

Next, we distinguish three classes of defect structures in PhCs, depicted in Fig. 2.4. They are treated differently within the Wannier function expansion. Therefore, each class is discussed in a separate subsection. Furthermore, the optimization of functional elements is handled separately. 


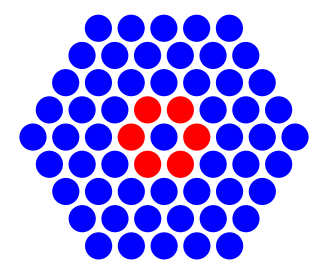

(a) cavity

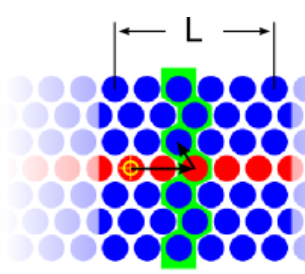

(b) waveguide

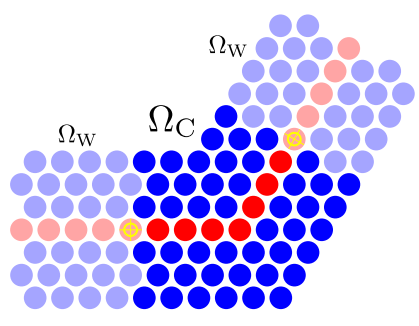

(c) functional element

Figure 2.4.: Classes of defect structures within a $\mathrm{PhC}$. We distinguish (a) cavities, (b) waveguides and (c) functional elements. Each class is treated differently within the Wannier function expansion.

\subsubsection{Calculation of Cavity Modes}

Cavities are 0-dimensional defect structures, composed of one or more point defects, i.e. they are isolated inside a $\mathrm{PhC}$ and therefore support localized eigenmodes with a discrete spectrum. The defect dielectric function of a cavity can be written as

$$
\delta \epsilon^{(\mathrm{cav})}(\vec{r})=\sum_{m=1}^{N_{\mathrm{p}}} \delta \epsilon_{m}\left(\vec{r}-\vec{R}_{m}\right),
$$

where $m$ runs over all $N_{p}$ point defects at their positions $\vec{R}_{m}$. Figure 2.4(a) shows a cavity composed of $N_{p}=6$ point defects.

We are interested in the eigenfrequencies and corresponding field distributions supported by a given configuration of point defects forming a photonic cavity. In this case, the wave equation states a generalized eigenvalue problem,

$$
\sum_{\beta}\left(M_{\alpha \beta}-\Lambda A_{\alpha \beta}\right) E_{\beta}=0 \quad \text { with } \quad M_{\alpha \beta}=C_{\alpha \beta}+D_{\alpha \beta},
$$

where the eigenvalue $\Lambda$ and the eigenvector $E_{\beta}$ are related to the frequency and field distribution of the cavity mode, respectively. The boundary conditions arise naturally by taking only sites in the vicinity of the defects (e.g., with distances up to $3 a$ to any defect) into account. This is appropriate, since for frequencies in the band gap of the underlying $\mathrm{PhC}$, fields decay exponentially into the bulk $\mathrm{PhC}$. Therefore, coefficients corresponding to Wannier functions far away from defect sites are zero for any valid solutions of the wave equation.

For the analysis of cavity modes, it is helpful to introduce a measure $V_{n}$ for the strength of the contributions of the individual Wannier function associated with band $n$,

$$
V_{n}=\sum_{\vec{R}}\left|E_{n \vec{R}}\right|^{2}
$$

Based on this quantity, it is possible to sort the important Wannier functions out from the less important ones in order to find an optimal set of Wannier functions. 


\subsubsection{Calculation of Waveguide Modes}

By arranging point defects periodically along a fixed lattice direction

$$
\vec{S}_{\mathrm{wg}}=\sum_{i} w_{i} \vec{a}_{i} \quad \text { with } \quad w_{i} \in \mathbb{N}_{0}
$$

one can create a line defect that possibly supports guided modes, i.e. modes that are localized in the transversal direction but propagate along the line defect. Properly designed, such $\mathrm{PhC}$ waveguides allow the efficient guiding of light for frequencies within a band gap.

As depicted in Fig. 2.4(b), the waveguide is split up into slices that represent its structural unit cells. The defect dielectric function can then be written as

$$
\delta \epsilon^{(\mathrm{wg})}(\vec{r})=\sum_{i=-\infty}^{\infty} \sum_{m=1}^{N_{\mathrm{p}}} \delta \epsilon_{m}\left(\vec{r}-\vec{R}_{m}^{(i)}\right) \quad \text { with } \quad \vec{R}_{m}^{(i)}=\underbrace{\vec{R}_{0}+i \vec{S}_{\mathrm{wg}}}_{\text {slice }}+\vec{\rho}_{m} .
$$

Here, the sum over $i$ addresses all slices and the sum over $m$ runs over all $N_{p}$ point defects inside one slice. Furthermore, the local lattice coordinate $\vec{\rho}$ within one slice was introduced.

To calculate the propagating and evanescent guided modes supported by the waveguide, we treat the frequency in the wave equation as a parameter only,

$$
\sum_{\beta} M_{\alpha \beta}(\omega) E_{\beta}=0 \quad \text { with } \quad M_{\alpha \beta}(\omega)=C_{\alpha \beta}+D_{\alpha \beta}-\Lambda(\omega) A_{\alpha \beta} .
$$

The Bloch theorem holds in the direction of periodicity of the waveguide. Therefore, we have to calculate solutions of (2.31) satisfying the Bloch boundary condition

$$
E\left(\vec{r}+\vec{S}_{\mathrm{wg}}\right)=\mathrm{e}^{\mathrm{i} \vec{k}(\omega) \vec{S}_{\mathrm{wg}}} E(\vec{r})
$$

and therefore characterized by a complex wave vector $\vec{k}(\omega)$. Since $\vec{k}$ must be parallel to $\vec{S}_{\mathrm{wg}}$, we can restrict ourselves to the calculation of scalars $k(\omega)$.

The waveguide modes are eigenmodes of a transfer matrix, which can be constructed as follows. We partition all relevant quantities according to the slicing of the waveguide introduced above. Therefore, we define a vector $\overrightarrow{\mathcal{E}}_{i}=\left\{E_{\alpha}\right\}_{i}$, which consists of the amplitudes of all Wannier functions centered in slice $i$. Similarly, we define the submatrices $\hat{\mathcal{M}}_{i, j}$ that contain all matrix elements $M_{\alpha \beta}$ that couple slice $i$ with slice $j$. Due to the translational invariance of the waveguide, we have $\hat{\mathcal{M}}_{i, j} \equiv \hat{\mathcal{M}}_{i-j}$. We assume that the coupling between slices separated by a distance exceeding some maximum distance $L a$ can be neglected,

$$
\hat{\mathcal{M}}_{i-j} \equiv 0 \text { for }\left|\vec{R}_{i}-\vec{R}_{j}\right| \geq L a .
$$

Putting the above definitions together, we arrive at the matrix equation

$$
\sum_{j=i-L}^{i+L} \hat{\mathcal{M}}_{i-j}(\omega) \overrightarrow{\mathcal{E}}_{j}=0
$$


which is equivalent to $(2.31)$. This equation can be rewritten in a transfer matrix form by defining $\vec{F}_{i}=\left\{\hat{\mathcal{E}}_{i}, \hat{\mathcal{E}}_{i+1}, \ldots, \hat{\mathcal{E}}_{i+2 L-1}\right\}$. As a result, we obtain

$$
\vec{F}_{i-1}=\hat{T}(\omega) \vec{F}_{i}
$$

where the transfer matrix $\hat{T}(\omega)=\left\{\hat{T}_{i, j}(\omega)\right\}$ has non-zero submatrix entries only for

$$
\begin{aligned}
\hat{T}_{1, j}(\omega) & =-\hat{\mathcal{M}}_{L}^{-1}(\omega) \hat{\mathcal{M}}_{L-j}(\omega) & & \text { for } j=1,2, \ldots, 2 L, \\
\hat{T}_{j+1, j}(\omega) & =1 & & \text { for } j=1,2, \ldots, 2 L-1 .
\end{aligned}
$$

Combining equation (2.35) with the Bloch theorem (2.32) allows us to identify the guided modes and corresponding wave vectors,

$$
\hat{T}(\omega) \vec{\phi}^{(\ell)}(\omega)=\mathrm{e}^{\mathrm{i} \vec{k}_{\ell}(\omega) \vec{S}_{\mathrm{wg}}} \phi^{(\ell)}(\omega) .
$$

Note that the resulting wave vectors $\vec{k}_{\ell}(\omega)$ are not necessarily real. In fact, most of them have a non-vanishing imaginary part, which means that the corresponding modes are evanescent in the direction of the waveguide. Therefore, equation (2.38) allows to calculate the propagating and evanescent guided modes of the waveguide. This is a very important prerequisite for the construction of the S-matrix of functional elements.

\subsubsection{Calculation of the S-matrix of Functional Elements}

The third class of defect structures are the functional elements (FEs). As the name already suggests, a functional element is designed to provide a specified functionality with respect to light guidance. A waveguide bend, for instance, tries to guide light around a corner without reflecting back too much light, whereas a beam splitter splits light incident from one waveguide equally into two outgoing waveguides. A functional element typically has several ports, i.e. connectors for waveguides, where light can come in and go out. As will be discussed in detail in chapter 3, the functionality of such a functional element can be characterized by its scattering matrix (S-matrix) containing all the information about the scattering of incoming field amplitudes into outgoing field amplitudes. ${ }^{3}$

The defect dielectric function of a functional element can be written as

$$
\delta \epsilon^{(\mathrm{fe})}(\vec{r})=\sum_{\vec{R}_{m} \in \Omega_{C}} \delta \epsilon_{m}\left(\vec{r}-\vec{R}_{m}\right)+\sum_{\vec{R}_{m} \in \Omega_{W}} \delta \epsilon_{m}\left(\vec{r}-\vec{R}_{m}\right) .
$$

The first term includes all defects located in the central region $\Omega_{C}$ of the $\mathrm{FE}$, while the second term comprises the defects located in the region $\Omega_{W}$ of the waveguides

\footnotetext{
${ }^{3}$ In fact, as the reader may have noticed, waveguides can be seen as functional elements as well. The reason to define them as a separate class of defect structures is their special importance for the scattering matrix formalism, since the guided modes of the waveguides connected to a functional element are used as incoming/outgoing channel modes for a specific frequency.
} 
connected to the ports. Note that the waveguides are now only semi-infinite, i.e. they start at the respective port positions and reach out to infinity in the outward direction. That means that the FE breaks the periodicity of the waveguides.

As for the calculation of waveguide guided modes in the previous section, we take a fixed frequency, which only acts as a parameter. The calculation of the S-matrix then involves the solution of the wave equation in Wannier function basis as a linear system of equations, written in the Wannier function basis as

$$
\sum_{\beta} M_{\alpha \beta}(\omega) E_{\beta}=0 \quad \text { with } \quad M_{\alpha \beta}(\omega)=C_{\alpha \beta}+D_{\alpha \beta}^{(\mathrm{fe})}-\Lambda(\omega) A_{\alpha \beta},
$$

where the indices $\alpha$ and $\beta$ run over the whole domain of the FE, including parts of the waveguides attached to the FE. We are interested in solutions to (2.40) under the constraint that there is a given field distribution coming in (namely only one incoming guided mode in one attached waveguide is excited). By analyzing the solution, i.e., the outgoing field distribution as a response to the incoming field, we can derive the amplitudes of outgoing guided modes and therefore the S-matrix for the FE. The details for this procedure are given in the following chapter in section 3.2.

\subsubsection{Optimization of Functional Elements}

As will become apparent in chapter 5 , it is often necessary to optimize FEs in a circuit such that they perform their individual operation best over a broad range of frequencies. For instance, an ideal bend would have no reflections for all frequencies, an ideal waveguide crossing would have no reflections and no crosstalk. Since in the scattering matrix framework (cf. chapter 3) each FE is fully characterized by its S-matrix, the relevant quantities to be optimized are all contained in the S-matrix, either as a single entry (e.g., reflectance at one port) or a combination of entries (e.g., sum of reflectance and crosstalk transmittance in a waveguide crossing). Therefore, the optimization procedure basically involves the calculation of the S-matrix for possible configurations of the FE.

In the first step, the parameter space has to be specified, in which the FE is varied. A reasonable restriction is to allow a limited set of lattice sites in the FE to take on different but discrete states. For instance, in a pore $\mathrm{PhC}$, a set of pores may be filled with different materials. This leads to a discrete and finite configuration space. However, the number of configurations $N_{\text {conf }}$ soon gets very large with an increasing number of point defects $N_{\text {sites }}$ and/or states per single point defect $N_{\text {states }}$ involved, since $N_{\text {conf }}=N_{\text {sites }}^{N_{\text {states }}}$. Therefore, a very efficient treatment of individual configurations is necessary, which is provided by the Wannier function expansion.

Once the configuration space is fixed, an appropriate optimization scheme is applied. Besides brute-force optimization (all configurations are checked), there exist a number of global optimization algorithms, such as simulated annealing [91] and genetic algorithms [92]. We do not go into details of the individual methods. Instead, we concentrate on the similarities between them and on resulting implications to the Wannier function expansion. 
In all optimization algorithms, the configuration space is scanned by evaluating a sequence of configurations and making decisions about which configurations are chosen next. In the Wannier function expansion, configurations only differ in their defect constellation, expressed by the defect overlap matrix $D_{\alpha \beta}$ in the wave equation (2.40). Therefore, the sequential evaluation of different configurations can be carried out by updating $D_{\alpha \beta}$ according to

$$
D_{\alpha \beta} \longrightarrow D_{\alpha \beta}+\delta D_{\alpha \beta},
$$

where $\delta D_{\alpha \beta}$ expresses the differences between two configurations. If the optimization algorithm uses sequences of "neighboring" configurations ${ }^{4}$, each update involves only changes of the state at one lattice site. Thus, the rank of $\delta D_{\alpha \beta}$ is small compared to the rank of the total system matrix. This allows us to avoid solving a full system of equations for each configuration separately. Instead, we can use a small-rank update to the inverse of the system matrix as follows. The system of equations ${ }^{5}$ to obtain the S-matrix of an updated configuration is given by

$$
(T+\delta D) \vec{X}=\vec{B} \quad \Longleftrightarrow \quad \vec{X}=(T+\delta D)^{-1} \vec{B}
$$

Note that in general, the inversion of the system matrix is much more expensive than the direct solution of the system. However, in the present case, in which $\delta D$ has small rank, the inverse of the updated system matrix, $(T+\delta D)^{-1}$, can be expanded in terms of the known inverse of the original system matrix, $T^{-1}$, using the Woodbury formula [93],

$$
\left(T+U V^{T}\right)^{-1}=T^{-1}-[T^{-1} U \underbrace{\left(1+V^{T} T^{-1} U\right)^{-1}}_{\text {small rank }} V^{T} T^{-1}] .
$$

Here, we have decomposed $\delta D=U V^{T}$ into two rectangular matrices $U$ and $V$ with dimensions $N \times P$ with $P \ll N$, while the system matrix $T$ has dimensions $N \times$ $N$. Correspondingly, the matrix $\left(1+V^{T} T^{-1} U\right)$, which has to be inverted in the update process, has small dimensions $P \times P$. Therefore, each update from one FE configuration to another is accelerated by the ratio of times it takes to invert a $P \times P$ matrix and to solve a $N \times N$ system of equations.

Clearly, the acceleration factor largely depends on the rank of $\delta D$, which increases with the number of Wannier functions involved in it. For larger ranks, the inversion of the "small" matrix, which has complexity $\mathcal{O}\left(N^{3}\right)$, gets more expensive than the solution of the larger system of equation, which scales with $\mathcal{O}\left(N^{2}\right)$. For instance, the rank of $\delta D$ is 294 if taking six Wannier functions on a domain of $7 \times 7$ lattice

\footnotetext{
${ }^{4}$ The term "neighboring configurations" is not well defined in general, but can be specified easily for each particular application. In our case, two configurations are "neighbors", if they can be transformed into each other by changing the state at only one lattice site. In simulated annealing, such an update scheme is inherent. For the brute-force optimization, there always exists a sequence of neighboring configurations that covers all possible configurations. In computer science, this sequence is known as a Gray code [93].

${ }^{5} \mathrm{~A}$ detailed derivation of the system of equations is given in section 3.2 in the next chapter.
} 
sites into account for an update of a single point defect. Compared to this, a system of $20 \times 20$ sites with six Wannier functions at each site has a system size of 2400 . For this case, small-rank updates are considerably faster than the solution of the full system of equations. However, if twenty-six Wannier functions are necessary, as is the case in chapter 5 , the solution of the full system of equation is more efficient. 


\section{Scattering Matrix Formalism}

In this chapter, we describe the basic concepts of the scattering matrix approach. We discuss in detail the method to obtain the scattering matrix of individual functional elements and how these individual $S$-matrices are combined to the circuit $S$-matrix. Furthermore, the importance of symmetries of functional elements is emphasized.

\subsection{Basic Concept}

In wave physics, the scattering of waves by a physical structure is of great importance. In the framework of scattering theory, complex amplitudes of outgoing waves (at $t \rightarrow \infty$ ) are related to complex amplitudes of incoming waves (at $t \rightarrow-\infty$ ) by the scattering matrix (S-matrix). Therefore, the S-matrix quantitatively characterizes a given physical structure with respect to its scattering properties. The incoming and outgoing waves are expressed in terms of channels. The channel modes are typically chosen in accord with the symmetries and boundary conditions of the system under consideration. They can, for instance, be plane waves (for planar structures) or spherical waves (for radially confined structures). To accurately describe the asymptotics of the scattering process, the channel modes should form a complete basis for the incoming and outgoing waves far away from the scatterer.

In our context, namely electromagnetic fields guided and scattered inside a $\mathrm{PhC}$ for frequencies in the band gap, light enters and leaves the structures only at clearly defined locations, the ports of the device. Waveguides are connected to these ports and the device acts as a scatterer for the incoming guided modes in these waveguides. Thus, the natural choice is to use these guided modes of the waveguides as channel modes in the framework of scattering theory. In this picture, the device is fully characterized by an S-matrix, which relates amplitudes of incoming to outgoing guided modes. We refer to this form of S-matrix as a guided-mode scattering matrix. This concept is depicted in Fig. 3.1.

The S-matrix relation can be formulated as

$$
\left(\begin{array}{c}
b_{1} \\
b_{2} \\
\vdots \\
b_{\ell} \\
\vdots
\end{array}\right)=\left(\begin{array}{ccccc}
S_{11} & S_{12} & \ldots & \ldots & \ldots \\
S_{21} & S_{22} & \ldots & \ldots & \ldots \\
\vdots & \vdots & \ddots & \ldots & \ldots \\
\vdots & \vdots & \vdots & S_{\ell \ell^{\prime}} & \ldots \\
\vdots & \vdots & \vdots & \vdots & \ddots
\end{array}\right)\left(\begin{array}{c}
a_{1} \\
a_{2} \\
\vdots \\
a_{\ell^{\prime}} \\
\vdots
\end{array}\right) \quad \text { or } \quad b_{\ell}=\sum_{\ell^{\prime}=1}^{N_{\mathrm{ch}}} S_{\ell \ell^{\prime}} a_{\ell^{\prime}},
$$

where $N_{\mathrm{ch}}$ is the number of channels and $a_{\ell}$ and $b_{\ell}$ are complex amplitudes of incoming and outgoing guided modes, respectively. The elements $S_{\ell \ell^{\prime}}$ of the S-matrix 


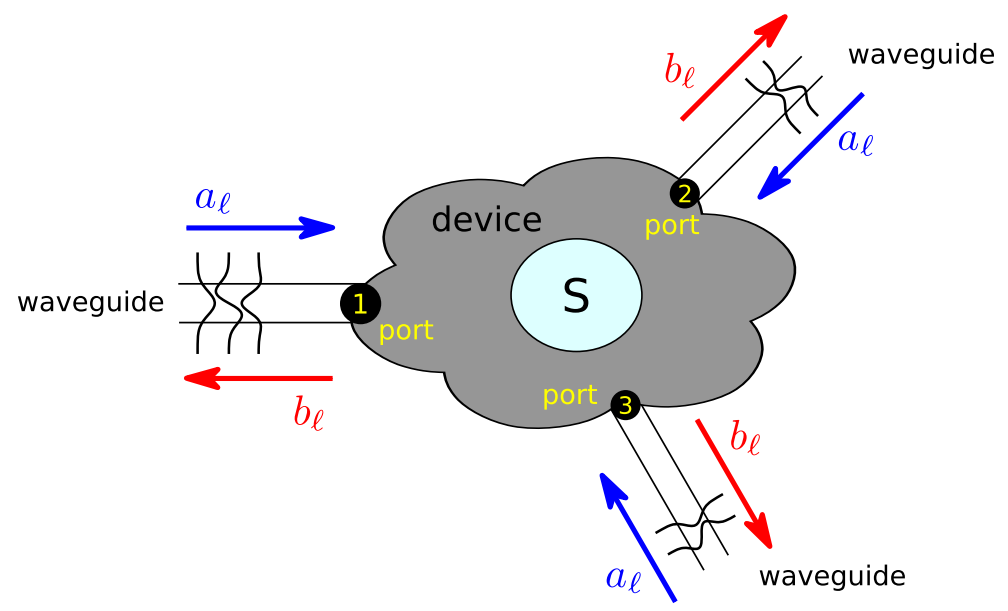

Figure 3.1.: Concept of the guided-mode scattering-matrix approach. A device is described by a scattering matrix $S$, which connects incoming (blue) to outgoing (red) guided-mode amplitudes, $a_{\ell}$ and $b_{\ell}$, in the waveguides connected to the ports of the device.

are complex quantities as well and contain information about the intensity (transmittances and reflectances) as well as the phase of the scattered field. Therefore, the S-matrix fully determines the outgoing field, $H^{\text {out }}(\vec{r})=\sum_{\ell} b_{\ell} G_{\ell}^{\text {out }}(\vec{r})$, for a given incoming field $H^{\text {in }}(\vec{r})=\sum_{\ell} a_{\ell} G_{\ell}^{\text {in }}(\vec{r})$, where $G_{\ell}^{\text {in/out }}(\vec{r})$ denote the incoming and outgoing guided modes, respectively.

Besides the computation of transmittances and reflectances of single devices, the S-matrix formalism allows to treat large-scale circuits that would otherwise be numerically expensive, if not impractical to treat. The basic idea is to split a large circuit into certain basic functional elements (FEs), such as bends or Y-splitters, and to interconnect them via waveguides of different length. This principle is depicted in Fig. 3.2. The S-matrices of the individual FEs can be computed with much less effort as compared to the full circuit S-matrix. Once they are obtained, the full S-matrix of the entire circuit is easily calculated for any lengths of the waveguides connecting the individual basic FEs. Thus, length-dependent characteristics of the circuit can be easily obtained. Due to this feature, the scattering matrix approach provides a very powerful design tool for large-scale $\mathrm{PhC}$ circuits.

\subsection{S-Matrix of Individual Functional Elements}

After the discussion of the general concept, we show in detail how to calculate the $\mathrm{S}$-matrix of a particular $\mathrm{FE}$ inside a $\mathrm{PhC}$ using a Wannier function expansion. We 


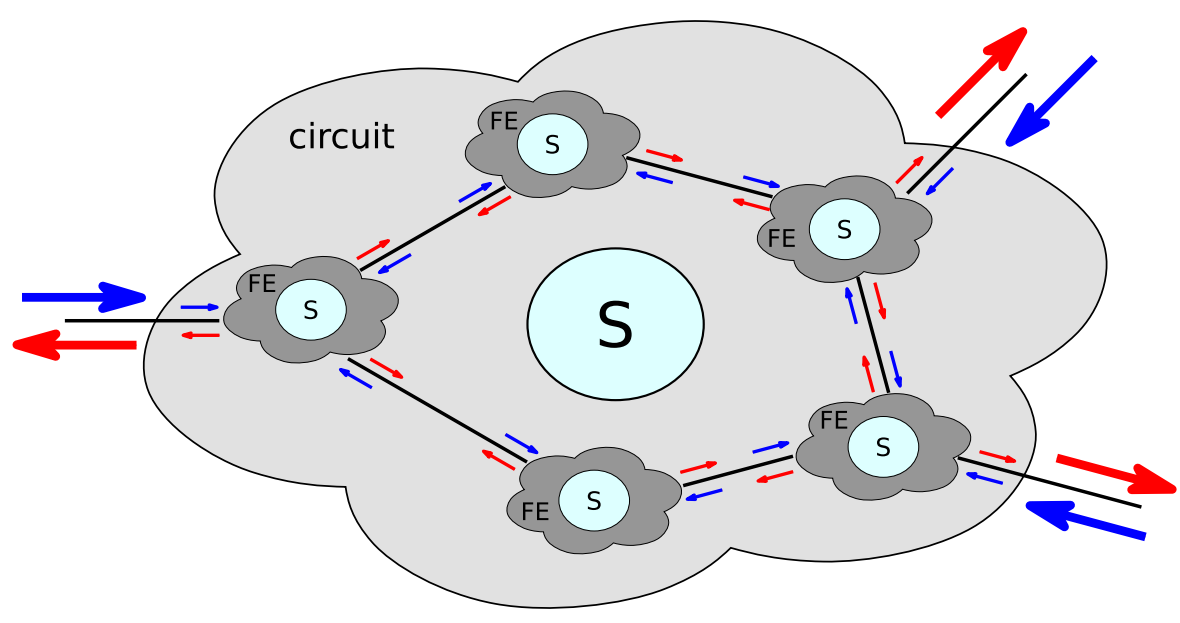

Figure 3.2.: Circuit description in the guided-mode scattering-matrix approach. The S-matrices of the individual FEs that compose the circuit are combined to the circuit S-matrix. There is no fundamental difference between the S-matrix of an FE and that of the circuit.

start with the wave equation in Wannier function basis, cf. Eq. (2.40),

$$
\sum_{\beta} M_{\alpha \beta}(\omega) H_{\beta}=0 \quad \text { for } \quad \alpha, \beta \in \Omega_{C} \cup \Omega_{W},
$$

where the composite indices $\alpha$ and $\beta$ cover the whole region of the FE. The frequency dependence of the matrix $M_{\alpha \beta}$ is omitted in the following for simplicity. Next, we divide the FE into two regions: the waveguiding region, $\Omega_{W}$ (union of all port regions), where the field can be described by incoming and outgoing guided modes, and the central region of the $\mathrm{FE}, \Omega_{C}$, where the scattering of incoming into outgoing modes takes place. These two regions are illustrated in Fig. 3.3. The guided modes in the waveguiding region $\Omega_{W}$ can either be propagating or evanescently decaying into or away from the device. They are consecutively labeled over all waveguides. Thus, in the case of identical ports, each of them having $n_{\ell}$ incoming and $n_{\ell}$ outgoing modes, the guided modes in port 1 that propagate or grow into the same direction are marked by $\ell=1, \ldots, n_{\ell}$, those in port 2 by $\ell=n_{\ell}+1, \ldots, 2 n_{\ell}$, and so on. The total number of channels counted over all ports is then given by $N_{\mathrm{ch}}=N_{\text {ports }} \cdot n_{\ell}$. This compact notation avoids an additional notational overhead related to summations over the ports of the FE. In (3.2), we separate the unknowns $H_{\beta}$ belonging to $\Omega_{C}$ from those belonging to $\Omega_{W}$,

$$
\sum_{\beta \in \Omega_{C}} M_{\alpha \beta} H_{\beta}+\sum_{\beta \in \Omega_{W}} M_{\alpha \beta} H_{\beta}=0 \quad \text { for } \quad \alpha \in \Omega_{C} \cup \Omega_{W} .
$$

The main objective is to compute the S-matrix, which relates incoming and outgoing guided mode amplitudes. Therefore our strategy is to replace the Wannier coefficients 


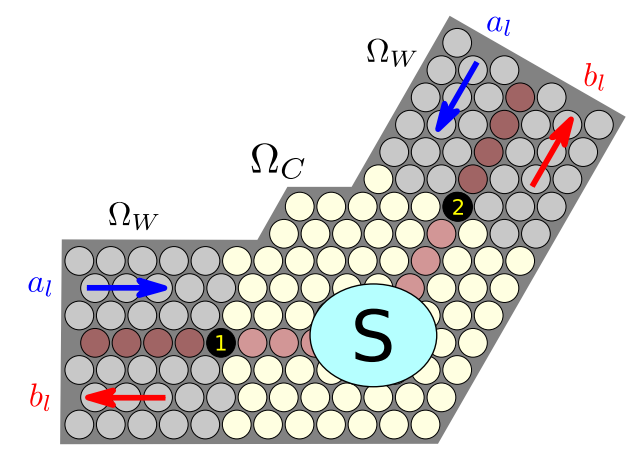

Figure 3.3.: Division of a given FE into two regions, $\Omega_{C}$ and $\Omega_{W}$. In the waveguiding region, $\Omega_{W}$ (shaded), the electromagnetic field can be described by incoming and outgoing guided modes and, therefore, the Wannier coefficients $H_{\beta}$ can be replaced by guided mode amplitudes, $a_{\ell}$ (incoming) and $b_{\ell}$ (outgoing), in that region.

$H_{\beta}$ in the waveguiding region by incoming and outgoing guided mode amplitudes $a_{\ell}$ and $b_{\ell}$, respectively. To begin with, we expand the field in one of the waveguide regions into guided modes $G_{l}^{\text {in }}(\vec{r})$ and $G_{l}^{\text {out }}(\vec{r}),{ }^{1}$

$$
H(\vec{r})=\sum_{l=1}^{N_{\mathrm{ch}}}\left(a_{l} G_{l}^{\mathrm{in}}(\vec{r})+b_{l} G_{l}^{\mathrm{out}}(\vec{r})\right) .
$$

The guided modes themselves can also be expanded into Wannier functions,

$$
G_{l}^{\text {in } / \text { out }}(\vec{r})=\sum_{\beta} G_{\beta l}^{\text {in } / \text { out }} W_{\beta}(\vec{r}) .
$$

By inserting (3.5) into (3.4) and comparing with $H(\vec{r})=\sum_{\beta} H_{\beta} W_{\beta}(\vec{r})$,

$$
H(\vec{r})=\sum_{\beta} \sum_{l=1}^{N_{\mathrm{ch}}}\left(a_{l} G_{\beta l}^{\mathrm{in}}+b_{l} G_{\beta l}^{\text {out }}\right) W_{\beta}(\vec{r}) \stackrel{!}{=} \sum_{\beta} H_{\beta} W_{\beta}(\vec{r}),
$$

we see that the Wannier coefficient $H_{\beta}$ in the waveguide region can be replaced by

$$
H_{\beta}=\sum_{l=1}^{N_{\mathrm{ch}}}\left(a_{l} G_{\beta l}^{\mathrm{in}}+b_{l} G_{\beta l}^{\mathrm{out}}\right) .
$$

\footnotetext{
${ }^{1}$ Note that since the FE destroys the periodicity of the waveguides along their respective direction, the guided modes are no longer exact solutions of the wave equation in the waveguide region $\Omega_{\mathrm{W}}$. Nevertheless, the FE contains a buffering region, resembling the waveguide, between the port and the defects that destroy the periodicity in the center. The fields scattered by the FE form the guided modes rapidly inside this buffering region, so that the guided modes can be considered to be good approximations to the exact solutions of the wave equation in the waveguide regions outside the buffer.
} 
The wave equation (3.3) can thus be written as

$$
\sum_{\beta \in \Omega_{C}} M_{\alpha \beta} H_{\beta}+\sum_{\beta \in \Omega_{W}} M_{\alpha \beta} \sum_{l=1}^{N_{\mathrm{ch}}}\left(a_{l} G_{\beta l}^{\mathrm{in}}+b_{l} G_{\beta l}^{\mathrm{out}}\right)=0 .
$$

With the definition of

$$
F_{\alpha l}^{\text {in/out }}:=\sum_{\beta \in \Omega_{W}} M_{\alpha \beta} G_{\beta l}^{\text {in/out }}
$$

and by separating incoming from outgoing amplitudes we get

$$
\sum_{\beta \in \Omega_{C}} M_{\alpha \beta} H_{\beta}+\sum_{l=1}^{N_{\mathrm{ch}}} F_{\alpha l}^{\mathrm{out}} b_{l}=-\sum_{l=1}^{N_{\mathrm{ch}}} F_{\alpha l}^{\mathrm{in}} a_{l} .
$$

The incoming amplitudes are placed on the right-hand side since they correspond to amplitudes we provide for the calculation. By contrast, we put the outgoing amplitudes, which are the unknowns to be solved for, on the left-hand side. We can write (3.10) in the matrix form

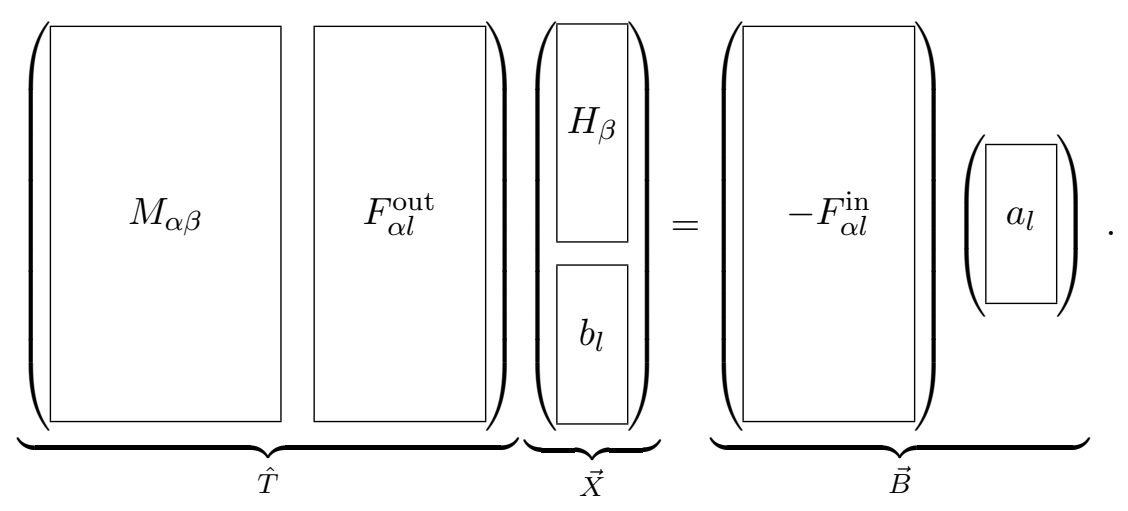

Finally, by setting $a_{l}=\delta_{l j}$ (i.e. only one incoming guided mode is excited) and solving (3.11) for $\vec{X}$ (i.e. for the outgoing amplitudes $b_{l}$ ), we obtain the $\mathrm{j}$-th column of the S-matrix,

$$
S_{l j} \equiv b_{l} \quad \text { for } \quad a_{l}=\delta_{l j} .
$$

If we solve (3.11) for several right hand sides, $a_{l}=\delta_{l j}, j=1 \ldots N_{\mathrm{ch}}$, we naturally get the full S-matrix of the FE.

As a side product, the solution vector $\vec{X}$ contains Wannier coefficients $H_{\beta}$ corresponding to the field distribution in the device region $\Omega_{C}$ for a given incoming guided mode.

\subsubsection{Reduced S-Matrix}

The waveguides connected to the ports of an FE usually support only a few propagating guided modes; the vast majority of guided modes is evanescent. These evanescent 
modes play an important role in the near field of structures that break the translational symmetry of the waveguide. In addition, they are necessary to correctly describe field components that evanescently decay away from defect structures. Therefore, we have to take them into account when computing the S-matrices of individual FEs.

On the other hand, outgoing evanescent field components die out very rapidly away from the FE and, therefore, have only negligible overlap with fields in a neighboring FE, provided that the FEs are sufficiently far separated. For the computation of the circuit S-matrix, it is, therefore, possible to work with reduced S-matrices of individual FEs, in which only the propagating modes are retained after the S-matrix has been computed with the help of all modes.

\subsection{Symmetries}

In this section, we consider various aspects related to symmetries of the structures we are interested in. We consider fundamental symmetries, such as energy conservation and reciprocity, as well as geometrical symmetries of certain functional elements. Finally, we will discuss the congruence of FEs, which is, strictly speaking, not a "symmetry" of the structures but which helps to relate S-matrices of different but related FEs. Exploiting symmetries and congruence of FEs may lead to a great alleviation of the computational effort necessary to treat large-scale circuits.

As an example, we will refer to the S-matrix of a general two-port device, which can be written as

$$
S=\left(\begin{array}{cc}
r & t \\
\bar{t} & \bar{r}
\end{array}\right)
$$

with the forward reflection and transmission coefficients $r$ and $t$ and the reverse coefficients $\bar{r}$ and $\bar{t}$, which, in general, can be matrices, if there is more than one propagating channel in the ports.

\subsubsection{Reciprocity}

Structures in which the dielectric permittivity tensor is symmetric and which do not have nonlinear response show a fundamental symmetry called reciprocity. This symmetry is found not only in electromagnetism but in many other fields, too, including particle scattering, Green's function methods and electrical network theory. Here, we give only a brief overview of the subject and refer the reader to the literature [94-96] for a more detailed discussion. A comprehensive survey on reciprocity in optics and a large list of further references can especially be found in a review article by Potton [97].

Reciprocity, in general, can be summarized in the sense that the positions of cause and effect are interchangeable:

If a current at point $\mathrm{A}$ generates a field at point $\mathrm{B}$, then the same current, placed at point $\mathrm{B}$, would produce exactly the same field in magnitude and 
phase at point $\mathrm{A}$, irrespective of the scattering and absorption taking place between point $\mathrm{A}$ and point $\mathrm{B}$.

For instance, antennae typically show exactly the same characteristics for transmission and reception. In the context of energy flow in a photonic circuit, reciprocity means that if energy is input via channel one and transmitted into channel two, the reverse energy flow from channel two to channel one would be exactly the same. This has the severe implication that it is not possible to build an optical isolator (the analogue of the diode in electronics) without breaking reciprocity. One needs non-reciprocal media, such as magneto-optical or nonlinear materials, to be able to construct an optical isolator.

On the other hand, reciprocity does not mean that reciprocal devices can not have different effects on waves in opposite directions. For example, a stack consisting of a linear polarizer combined with a quarter wave plate produces a linearly polarized output for one direction of illumination and circularly polarized output for the other direction of illumination. Nevertheless, it is still reciprocal. Reciprocity always connects channels in pairs, irrespective of other channels, so the resulting functionality of devices does not need to be symmetric at all for reciprocal devices.

There exist many different formulations of reciprocity, even in electromagnetism. Each of them applies to different aspects of the systems under consideration. In electromagnetism, the vector nature (polarization) of the fields has to be taken into account, and thus there is even more space for differentiation, for instance considering polarizers and chiral materials. We do not go into more details here, since it is not necessary for our discussion below. Instead, we would like to point out that the reciprocity principle can be obtained in a very general form [94], only assuming Maxwell's equations in linear form and symmetric dielectric permittivity tensors of the constituent materials. So the distinction of whether a given structure is reciprocal or not is basically independent of the structure geometry. Therefore, geometric asymmetries, chirality or similar properties do not destroy the reciprocity of a structure. This, for instance, implies that it is on principle not possible to design a Faraday rotator by using reciprocal materials only, even if it has complicated chiral structure.

In the context of the guided-mode scattering matrix formalism, reciprocity implies that the S-matrix in (3.1) is symmetric, i.e.,

$$
S_{\ell \ell^{\prime}}=S_{\ell^{\prime} \ell}
$$

or, for the two-port example in (3.13),

$$
\bar{t}=t .
$$

This reduces the number of independent matrix elements significantly, since only the upper (or lower) triangle of the S-matrix has to be considered. Therefore, the number of scattering parameters that fully characterize a device is also reduced for a large class of structures.

In addition, reciprocity allows to make precise, but somewhat non-intuitive, statements about device performance. An example of such a case is shown in Fig. 3.4. 


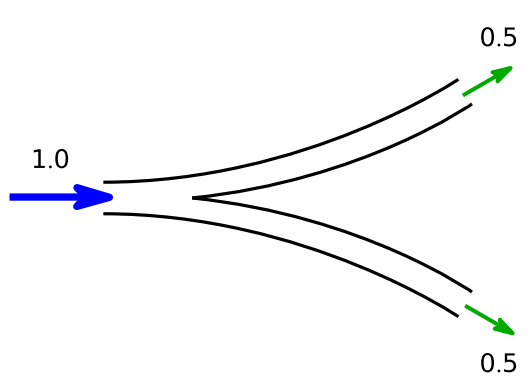

splitter

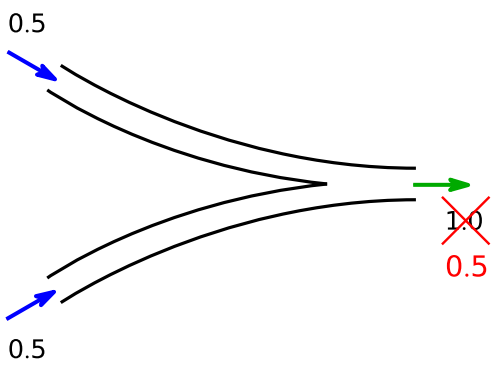

combiner

Figure 3.4.: Example for the strong implications of reciprocity. The ideal splitter on the left can not be used as an efficient combiner. Reciprocity determines that only half of the input intensity is output in the combined port.

An ideal splitter, which splits an incoming beam of intensity 1.0 (arbitrary units) into two output beams of intensity 0.5 , may intuitively be seen as a candidate for an ideal combiner when operated in reverse direction. But instead of fully merging two incoming beams of intensities 0.5 into one beam of intensity 1.0, reciprocity requires that each incoming beam must be attenuated by a factor of one half. Therefore, the resulting combined beam has an intensity of only 0.5 , the splitter can not be used as an efficient combiner. This limitation for combiners is fundamental, unless reciprocity is broken by the underlying material.

\subsubsection{Energy conservation}

For structures built from media in which the energy is conserved the S-matrix is unitary. For a two-port device with the S-matrix

$$
S=\left(\begin{array}{ll}
r & t \\
\bar{t} & \bar{r}
\end{array}\right),
$$

the unitarity of $S$ means that rows and columns sum up to one, which, more precisely, is formulated as

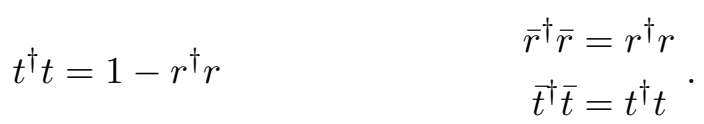

Transmission coefficients are, therefore, complementary to reflection coefficients, and the reverse coefficients $\bar{r}$ and $\bar{t}$ are equal to the forward coefficients $r$ and $t$, up to unitary transformations (phase factors).

\subsubsection{Geometric Symmetries}

In many cases, FEs exhibit additional geometric symmetries, i.e., they are invariant under spatial symmetry operations.

As with the fundamental symmetries discussed above, such symmetries are reflected in the S-matrix of the FE. They further reduce the number of independent 

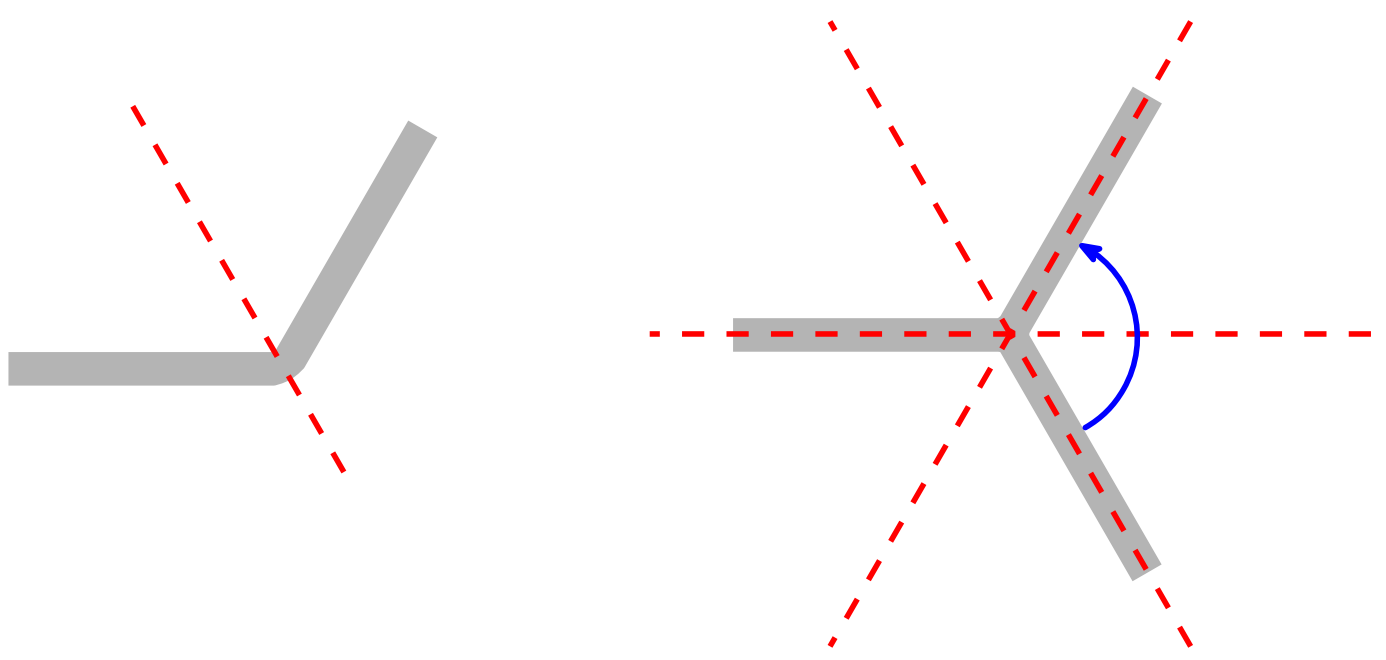

Figure 3.5.: Geometric symmetries of certain FEs, schematically illustrated for a bend (left picture) and a Y-splitter (right picture). The geometric symmetries are reflected in symmetries of the S-matrix and reduce the number of independent matrix elements of the S-matrix.

matrix elements. Fig. 3.5 schematically shows two examples, a waveguide bend and a Y-splitter. The bend is symmetric with respect to one mirror reflection (symmetry group $\mathcal{D}_{1}$ ). This leads to an S-matrix of the form

$$
S_{\text {bend }}=\left(\begin{array}{ll}
r & t \\
t & r
\end{array}\right) \text {, }
$$

which is reduced to only two independent matrix blocks, $r$ and $t$, one reflection and one transmission block, respectively. While the two identical transmission blocks can already be interpreted as a consequence of reciprocity, the equality of the two reflection blocks, even for cases without energy conservation, results exclusively from the geometric symmetry.

The Y-splitter in Fig. 3.5 belongs to the symmetry group $\mathcal{D}_{3}$, which includes all rotations about $120^{\circ}$ (cyclic group $\mathcal{C}_{3}$ ) plus three mirror reflections. The S-matrix takes the form

$$
S_{\text {splitter }}=\left(\begin{array}{ccc}
r & t & t \\
t & r & t \\
t & t & r
\end{array}\right) \text {, }
$$

with only two independent blocks, $r$ and $t$. Again, the equality of the corresponding transmission blocks above and below the diagonal is already explained by reciprocity, but the equality of all reflection and transmission blocks, $r$ and $t$, is a consequence of the high geometrical symmetry of the Y-splitter. 


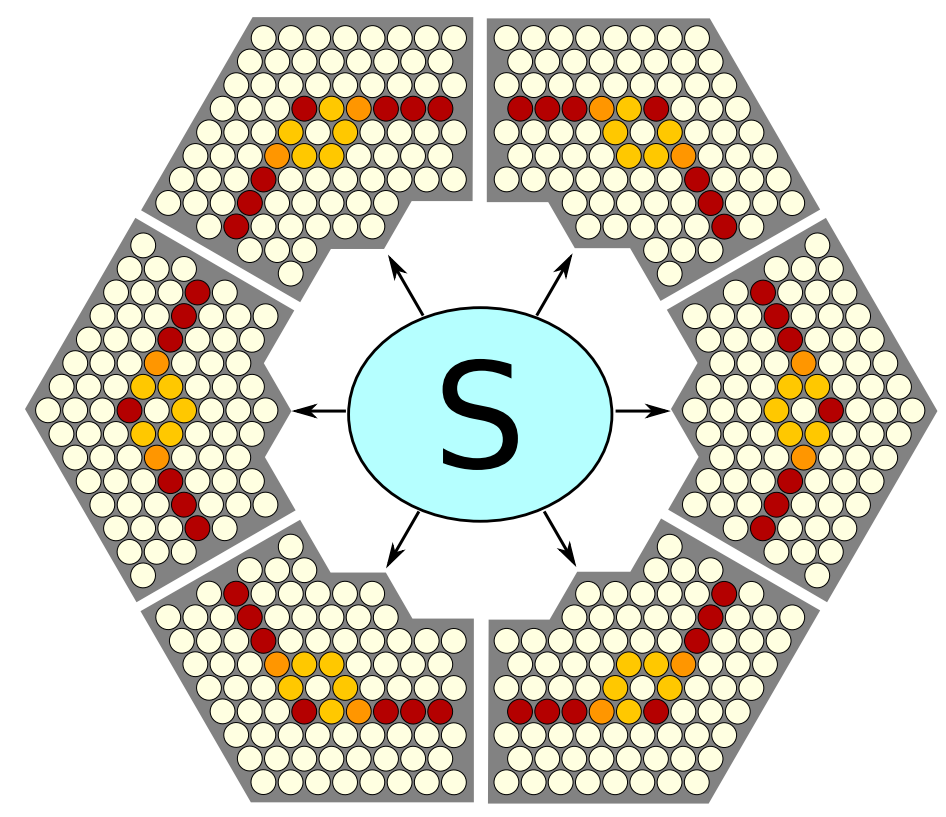

Figure 3.6.: Congruence of FEs: All six bends shown above are characterized by the same S-matrix, provided that the guided modes of the waveguides connected to them, which also appear in different orientations, are mapped exactly onto each other.

\subsubsection{Congruence of Functional Elements}

The S-matrix of an FE depends on the position of the ports and the scatterers in the $\mathrm{FE}$ relative to each other, but it does not depend on the orientation of the $\mathrm{FE}$ as a whole (including ports) within the background. For instance, in a $2 \mathrm{D} \mathrm{PhC}$ with triangular lattice, a bend can be inscribed in six orientations that all exhibit the same S-matrix. This is depicted in Fig. 3.6.

In general, we can state that all FEs that are congruent (i.e., that can be transformed into each other by translations, rotations, and mirror reflections) possess the same S-matrix, provided that the guided modes in the ports of two congruent FEs are mapped exactly onto each other by the congruence transformation. This condition is fulfilled in the example in Fig. 3.6, since the waveguide connected to the ports of the bends provides exactly one propagating guided mode, which has even symmetry under a mirror reflection at the plane along the waveguide (cf. chapter 5 for further discussion of that particular model system). Thus, the guided mode is mapped onto itself for any congruence transformation including mirror reflections. In section 5.2.1, we will discuss a counter example, the coupling section waveguide of a directional coupler, in which the guided modes are not mapped exactly onto each other by some congruence transformations. In such a case, the S-matrices of congruent FEs may differ in the ordering of rows and columns.

In summary, by carefully taking into account the congruence of FEs, we can save a 


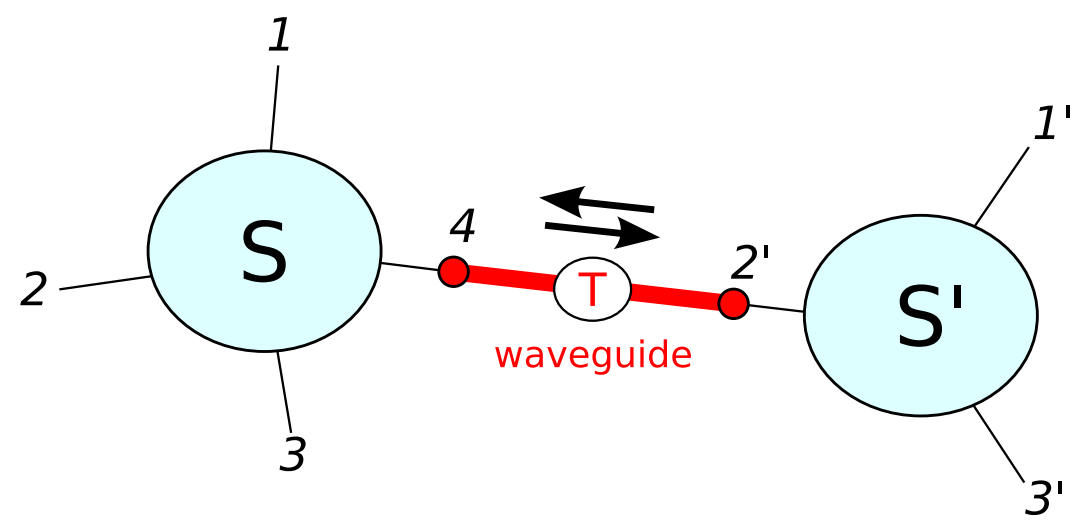

Figure 3.7.: Illustration of how to connect two individual FEs with S-matrices $S$ and $S^{\prime}$ to form a complex FE. The interior ports 4 and $2^{\prime}$ are eliminated while the exterior ports $1,2,3,1^{\prime}$, and $3^{\prime}$ form the new ports of the combined FE. The length of the waveguide connecting the two FEs appears as a phase factor in the switching matrix $T$. In addition, the switching matrix accounts for potential mode mismatch if the waveguides at ports 4 and $2^{\prime}$ are different.

major amount of computational effort when analyzing a complex circuit. For each FE, the S-matrix needs to be calculated only in one specific orientation. The S-matrices for all other orientations are equal to that S-matrix or can be derived according to the simple congruence rules described above.

\subsection{S-Matrix Combination}

Once we have the S-matrices of all individual FEs, we need to combine them to the overall circuit S-matrix. In this process, not only the scattering of radiation by FEs has to be taken into account but also the phase shifts in the waveguide connections of variable length between the FEs. Phase-sensitive features, such as Fabry-Perot resonances, have to be treated carefully in the formalism.

The S-matrix combination is done step-by-step for each connection in the circuit. The general procedure for resolving a single connection between two FEs is as follows. Each individual FE has a number of ports, where each port exhibits a certain number of channels, i.e., modes supported by the corresponding waveguide. When connecting two FEs, the resulting combined FE inherits the exterior ports of the two FEs, i.e., the ports that are not involved in the connection. The interior ports, i.e., those ports that are part of the connection between the individual FEs, are eliminated in such a way that the coupling of the two FEs is correctly accounted for in the combined Smatrix. Fig. 3.7 illustrates the general procedure of combining S-matrices of different FEs. To be more specific, we formulate the following details of calculations according 
to the particular case depicted in this figure.

The actual calculation scheme to obtain the combined S-matrix can be derived from the block-diagonal system of equations

$$
\left(\begin{array}{c}
b_{1} \\
b_{2} \\
b_{3} \\
b_{4} \\
b_{1^{\prime}} \\
b_{2^{\prime}} \\
b_{3^{\prime}}
\end{array}\right)=\left(\begin{array}{ccccccc}
S_{11} & S_{12} & S_{13} & S_{14} & 0 & 0 & 0 \\
S_{21} & S_{22} & S_{23} & S_{24} & 0 & 0 & 0 \\
S_{31} & S_{32} & S_{33} & S_{34} & 0 & 0 & 0 \\
S_{41} & S_{42} & S_{43} & S_{44} & 0 & 0 & 0 \\
0 & 0 & 0 & 0 & S_{1^{\prime} 1^{\prime}}^{\prime} & S_{1^{\prime} 2^{\prime}}^{\prime} & S_{1^{\prime} 3^{\prime}}^{\prime} \\
0 & 0 & 0 & 0 & S_{2^{\prime} 1^{\prime}} & S_{2^{\prime} 2^{\prime}} & S_{2^{\prime} 3^{\prime}} \\
0 & 0 & 0 & 0 & S_{3^{\prime} 1^{\prime}}^{\prime} & S_{3^{\prime} 2^{\prime}}^{\prime} & S_{3^{\prime} 3^{\prime}}^{\prime}
\end{array}\right)\left(\begin{array}{c}
a_{1} \\
a_{2} \\
a_{3} \\
a_{4} \\
a_{1^{\prime}} \\
a_{2^{\prime}} \\
a_{3^{\prime}}
\end{array}\right)
$$

with the additional constraint

$$
\left(\begin{array}{c}
b_{4} \\
b_{2^{\prime}}
\end{array}\right)=\underbrace{\left(\begin{array}{cc}
0 & G \\
G^{T} & 0
\end{array}\right)}_{=: T_{S}}\left(\begin{array}{c}
a_{4} \\
a_{2^{\prime}}
\end{array}\right),
$$

which accounts for the connection of ports 4 and $2^{\prime}$. In the typical case of an ideal connection without mode mismatch and perfectly corresponding incoming and outgoing modes in the two connected ports, the matrix $G$ is diagonal and contains only phase factors corresponding to the guided-modes' phase shifts over the length of the waveguide,

$$
G=\left(\begin{array}{lll}
\ddots & & 0 \\
& \operatorname{expi} k_{\ell} L & \\
0 & & \ddots
\end{array}\right) .
$$

Here, $k_{\ell}$ is the wave vector of the $\ell$-th guided mode and $L$ is the length of the connecting waveguide.

We rearrange (3.20) so that the exterior and interior ports are separated,

$$
\left(\begin{array}{l}
b_{1} \\
b_{2} \\
b_{3} \\
b_{1^{\prime}} \\
b_{3^{\prime}} \\
b_{4} \\
b_{2^{\prime}}
\end{array}\right)=\left(\begin{array}{ccccc|cc}
S_{11} & S_{12} & S_{13} & 0 & 0 & S_{14} & 0 \\
S_{21} & S_{22} & S_{23} & 0 & 0 & S_{24} & 0 \\
S_{31} & S_{32} & S_{33} & 0 & 0 & S_{34} & 0 \\
0 & 0 & 0 & S_{1^{\prime} 1^{\prime}}^{\prime} & S_{1^{\prime} 3^{\prime}}^{\prime} & 0 & S_{1^{\prime} 2^{\prime}}^{\prime} \\
0 & 0 & 0 & S_{3^{\prime} 1^{\prime}}^{\prime} & S_{3^{\prime} 3^{\prime}}^{\prime} & 0 & S_{3^{\prime} 2^{\prime}}^{\prime} \\
\hline S_{41} & S_{42} & S_{43} & 0 & 0 & S_{44} & 0 \\
0 & 0 & 0 & S_{2^{\prime} 1^{\prime}}^{\prime} & S_{2^{\prime} 3^{\prime}}^{\prime} & 0 & S_{2^{\prime} 2^{\prime}}^{\prime}
\end{array}\right)\left(\begin{array}{c}
a_{1} \\
a_{2} \\
a_{3} \\
a_{1^{\prime}} \\
a_{3^{\prime}} \\
a_{4} \\
a_{2^{\prime}}
\end{array}\right),
$$

and introduce composite symbolic indices, 'e' for exterior and 'i' for interior ports to write (3.23) and (3.21) in a more compact form,

$$
\left(\begin{array}{c}
b_{\mathrm{e}} \\
b_{\mathrm{i}}
\end{array}\right)=\left(\begin{array}{cc}
S_{\mathrm{e}, \mathrm{e}} & S_{\mathrm{e}, \mathrm{i}} \\
S_{\mathrm{i}, \mathrm{e}} & S_{\mathrm{i}, \mathrm{i}}
\end{array}\right)\left(\begin{array}{c}
a_{\mathrm{e}} \\
a_{\mathrm{i}}
\end{array}\right) \quad \text { and } \quad b_{\mathrm{i}}=T_{S} a_{\mathrm{i}} .
$$


Here, $a_{\mathrm{e}} \equiv\left(a_{1}, a_{2}, a_{3}, a_{1^{\prime}}, a_{3^{\prime}}\right), a_{\mathrm{i}} \equiv\left(a_{4}, a_{2^{\prime}}\right), b_{\mathrm{e}} \equiv\left(b_{1}, b_{2}, b_{3}, b_{1^{\prime}}, b_{3^{\prime}}\right)$, and $b_{\mathrm{i}} \equiv\left(b_{4}, b_{2^{\prime}}\right)$. The matrices $S_{\mathrm{e}, \mathrm{e}}, S_{\mathrm{e}, \mathrm{i}}, S_{\mathrm{i}, \mathrm{e}}$ and $S_{\mathrm{i}, \mathrm{i}}$ can be easily extracted from (3.23) by direct comparison. Formally, (3.24) is a system of three equations,

$$
\begin{aligned}
& b_{\mathrm{e}}=S_{\mathrm{e}, \mathrm{e}} a_{\mathrm{e}}+S_{\mathrm{e}, \mathrm{i}} a_{\mathrm{i}} \\
& b_{\mathrm{i}}=S_{\mathrm{i}, \mathrm{e}} a_{\mathrm{e}}+S_{\mathrm{i}, \mathrm{i}} a_{\mathrm{i}} \\
& b_{\mathrm{i}}=\quad T_{S} a_{\mathrm{i}} \text {, }
\end{aligned}
$$

in which the two interior composite vectors $a_{i}$ and $b_{i}$ can be eliminated. Inserting (3.27) into (3.26) and solving for $a_{\mathrm{i}}$ leads to

$$
a_{\mathrm{i}}=\left(T_{S}-S_{\mathrm{i}, \mathrm{i}}\right)^{-1} S_{\mathrm{i}, \mathrm{e}} a_{\mathrm{e}} .
$$

This can again be inserted into (3.25) to yield

$$
b_{\mathrm{e}}=\left[S_{\mathrm{e}, \mathrm{e}}+S_{\mathrm{e}, \mathrm{i}}\left(T_{S}-S_{\mathrm{i}, \mathrm{i}}\right)^{-1} S_{\mathrm{i}, \mathrm{e}}\right] a_{\mathrm{e}} .
$$

Now we can identify the combined S-matrix to be

$$
S_{\text {combined }}=S_{\mathrm{e}, \mathrm{e}}+S_{\mathrm{e}, \mathrm{i}}\left(T_{S}-S_{\mathrm{i}, \mathrm{i}}\right)^{-1} S_{\mathrm{i}, \mathrm{e}} .
$$

This expression, though derived for the particular example shown in Fig. 3.7, represents the general scheme for combining S-matrices of individual FEs. 


\section{Accuracy and Convergence}

Before using the Wannier function based scattering matrix approach presented conceptually in the previous chapters to design large-scale PhC circuits, we examine the accuracy and convergence of the approach when applied to a number of test cases for different host PhCs and polarizations. The results for cavity mode frequencies and waveguide dispersions are compared to supercell calculations done with the MIT Photonic Bands (MPB) package [63]. The S-matrix method is checked for self-consistency by comparing calculations of relatively small composed circuits, such as double bends or simple Mach-Zehnder interferometers, which can be calculated both as one block and as a combination of basic FEs.

\subsection{Model Systems}

To get an overview of the capabilities of the Wannier function approach for 2D PhCs, we consider two different model systems that somewhat represent the huge variety of host PhCs for both polarizations, $E$ - and $H$-polarization:

Model system $1 E$-polarized radiation in a square lattice of circular silicon rods with radius $r=0.18 a$. Depicted in Fig. 4.1.

Model system $2 \mathrm{H}$-polarized radiation in a triangular lattice of air holes with radius $r=0.45 a$ in silicon. Depicted in Fig. 4.2.

The dielectric permittivity of silicon is always assumed to be $\epsilon_{\mathrm{Si}}=12.0$, while air has a dielectric constant of $\epsilon_{\text {air }}=1.0$.

The two systems basically differ in their topology (rods vs. holes) and the polarization used ( $E$ vs. $H)$. The choice of radii was governed by the existence of large band gaps for the respective polarization. In Figs. 4.1 and 4.2, the band structures for the two systems are shown and the relevant band gaps are identified, which define the operational frequency range for cavities, waveguides and FEs within the host $\mathrm{PhCs}$.

For each model system, a finite number of Wannier functions per lattice site have been constructed from the Bloch modes of the system. We will show that the number of Wannier functions necessary to get converged results may vary strongly; it depends on the system (host $\mathrm{PhC}$ and polarization) as well as the defect structure that has to be modeled. Since the construction of well localized and symmetric Wannier functions is highly non-trivial, we are limited to a maximum number of twenty-three (model system 1) and thirty-eight (model system 2) Wannier functions. Within these bounds, the "completeness" of the Wannier function basis will be checked. The two sets of Wannier functions are listed in Appendices A and B, respectively. 

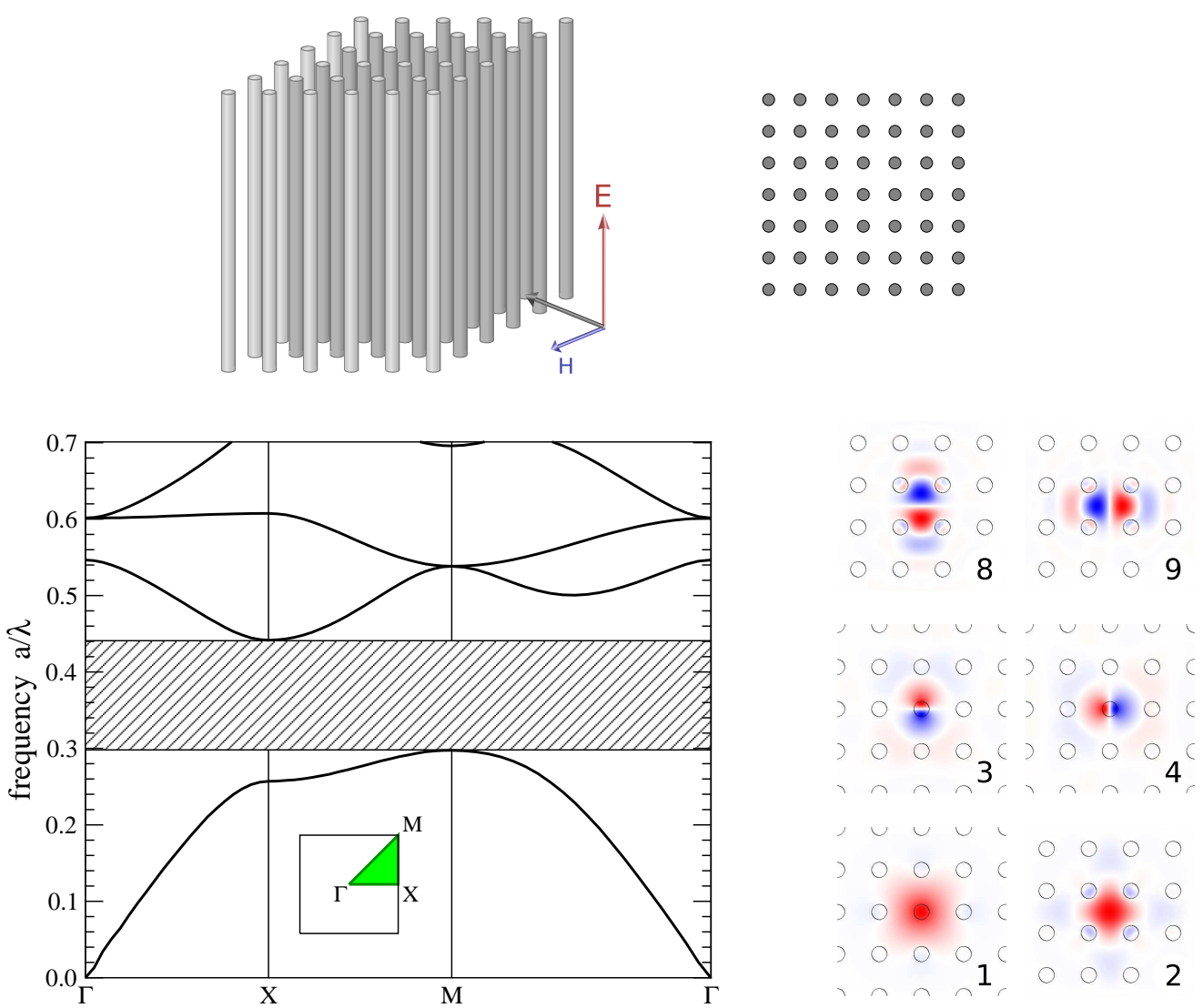

Figure 4.1.: Model system 1: square lattice of cylindrical silicon rods with radius $r=0.18 a$ for $E$-polarized radiation. The band structure shows the relevant band gap between the first and second band that is used as operational frequency range to be used later on. On the right hand side, six Wannier functions are plotted. For a complete list of all available twenty-three Wannier functions, see appendix A.

\subsubsection{Auxiliary Wannier Functions}

In some cases discussed in the following sections, it is not possible to achieve converged results with the finite number of Wannier functions produced for the original $\mathrm{PhC}$. Due to the completeness of the Wannier function basis, this primarily means that more Wannier functions are necessary and would have to be generated from higher bands. Since the construction of Wannier functions out of entangled bands can be very cumbersome, and the entanglement typically gets worse for higher bands, the production of more Wannier functions is not a realizable option, though.

Instead, it is sometimes possible to identify other sets of localized functions that can be used to complement the basis of the original Wannier functions. If a defect structure is built up of point defects supporting modes that can not be modeled by the original Wannier functions, these fields at the point defects can be much better 

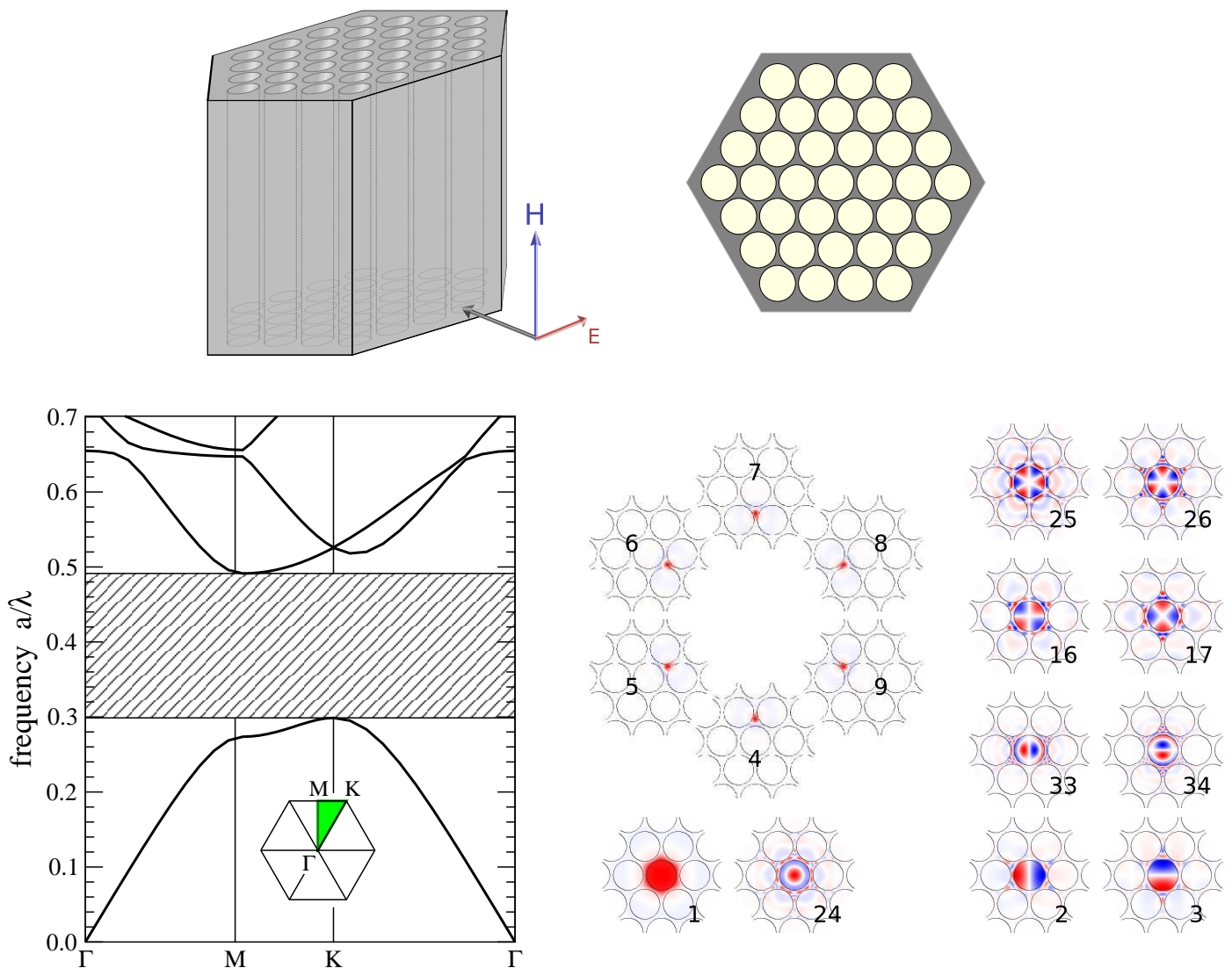

Figure 4.2.: Model system 2: triangular lattice of cylindrical air holes with radius $r=0.45 a$ in silicon for $H$-polarized radiation. The band gap between the first and second band prescribes the operational frequency range to be used later on. On the right hand side, a selection of Wannier functions is plotted. For a complete list of all available thirty-eight Wannier functions, see appendix B.

represented by the Wannier functions of a $\mathrm{PhC}$ topologically identical to the original $\mathrm{PhC}$, but consisting of a periodic arrangement of such point defects. These auxiliary Wannier functions live on the same direct lattice as the original Wannier functions, so they can be easily added to the original Wannier function basis to complement it. In addition, they contain all the information about symmetries of the lattice.

Note that in general, the resulting function basis of original and auxiliary Wannier functions is no longer orthonormal. In addition, the choice to use Wannier functions for a different $\mathrm{PhC}$ as a complement is somehow arbitrary. We could just as well use defect modes or any other set of localized functions related to the respective point defects. Nonetheless, auxiliary Wannier functions can, at least in theory, be generated in an arbitrary number. Furthermore, they represent a natural way of generalizing the Wannier function approach into the direction of structures consisting of different 


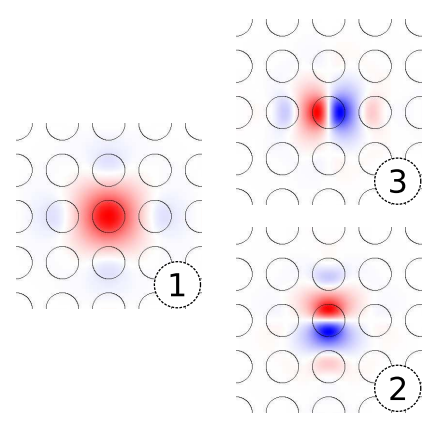

(a) $r=0.35 a$

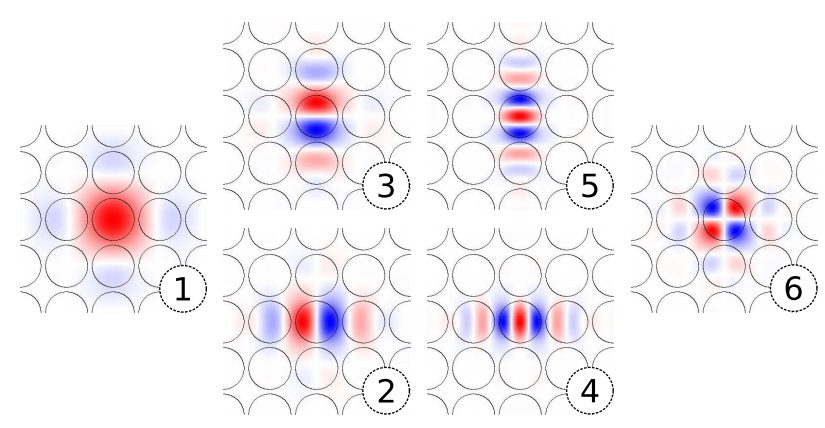

(b) $r=0.45 a$

Figure 4.3.: Sets of auxiliary Wannier functions for model system 1. They correspond to a square lattice of silicon rods with radius (a) $r=0.35 a$ and (b) $r=0.45 a$, respectively.
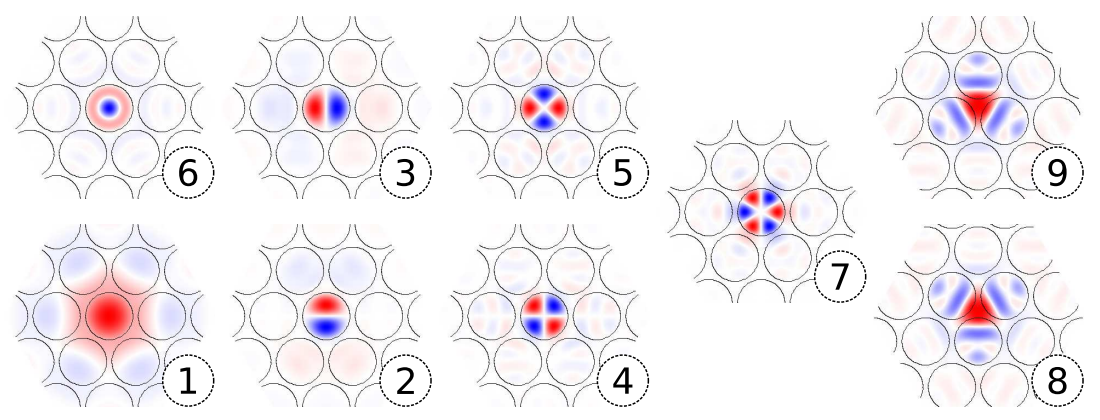

Figure 4.4.: Set of auxiliary Wannier functions for model system 2, corresponding to the inverse system, i.e., a triangular lattice of silicon rods with radius $r=0.45 a$.

domains, such as interfaces between two different PhCs [98].

It is important to keep in mind, that the task of the auxiliary Wannier functions is to improve convergence. The physics, however, is contained in the original Wannier functions. Therefore, it is favorable, and often advisable, to add auxiliary Wannier functions only in a very narrow region around the corresponding point defects, i.e., only at the defect site itself or including direct neighbor sites.

For the sake of clarity, we move up the list of concrete sets of auxiliary Wannier functions used later on. The two sets for model system 1, corresponding to a square lattice of silicon rods of radii $r=0.35 a$ and $r=0.45 a$, respectively, are depicted in Fig. 4.3. The single set for model system 2, corresponding to the inverse system, i.e., a triangular lattice of silicon rods of radius $r=0.45 a$ is shown in Fig. 4.4.

\subsection{Cavity Modes}

As a first check for the accuracy and convergence of the Wannier function expansion, we calculate modes for different cavities in both model systems. We examine cavities 


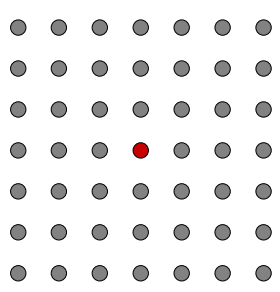

(a)

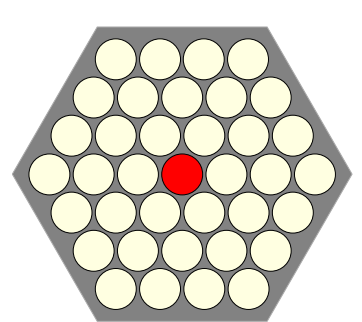

(d)

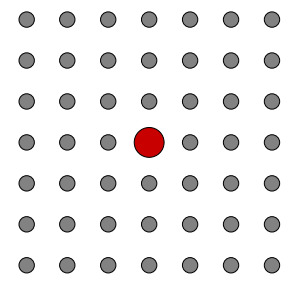

(b)

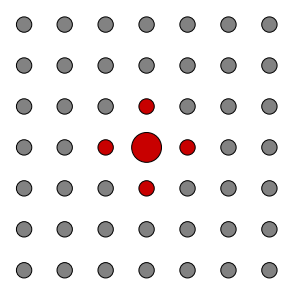

(c)

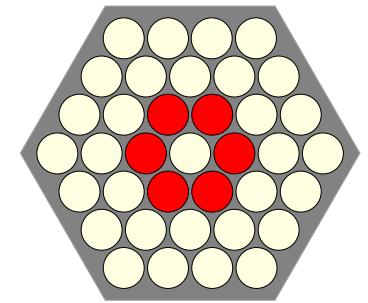

(e)

Figure 4.5.: Cavity types: model system 1: (a) variable dielectric permittivity of a single rod, (b) variable radius of a single, and (c) cavity composed of several defect rods. Cavity types in model system 2: (d) variable dielectric permittivity of a single hole (single-pore infiltration), and (e) ring cavity composed of six filled pores.

formed by a single point defect and cavities composed of several point defects. The resulting eigenfrequencies are compared to converged MPB supercell calculations, which are taken as reference solutions. Fig. 4.5 shows an overview of the cavities discussed in the following.

\subsubsection{Model System 1}

For model system 1, we discuss three different cavities depicted in the sub-figures (a) to (c) of Fig. 4.5. The first two cavities investigated will consist of a single rod with changed dielectric permittivity or with changed radius, respectively. Then we will examine a more complex cavity, in which those two kinds of point defects are mixed.

Variable Defect Rod Permittivity: We start with a single point defect created by changing the dielectric permittivity $\epsilon_{\text {def }}$ of a single rod, Fig. 4.5(a). The defect permittivity $\epsilon_{\text {def }}$ is varied between 1.0 and 50.0. The resulting cavity modes are plotted in Fig. 4.6. We get a monopole mode for $\epsilon_{\text {def }}$ smaller than $\epsilon_{\mathrm{Si}}=12.0$, the permittivity of the surrounding rods, and a twofold-degenerate dipole mode for $\epsilon_{\text {def }}$ larger than 12.0. The monopole mode evolves out of the lower band with decreasing $\epsilon_{\text {def }}$, while the dipole modes are pushed out of the upper band when increasing $\epsilon_{\text {def }}$.

To check the accuracy and convergence of the Wannier function results, we first performed the calculation with all twenty-three Wannier functions available for model 

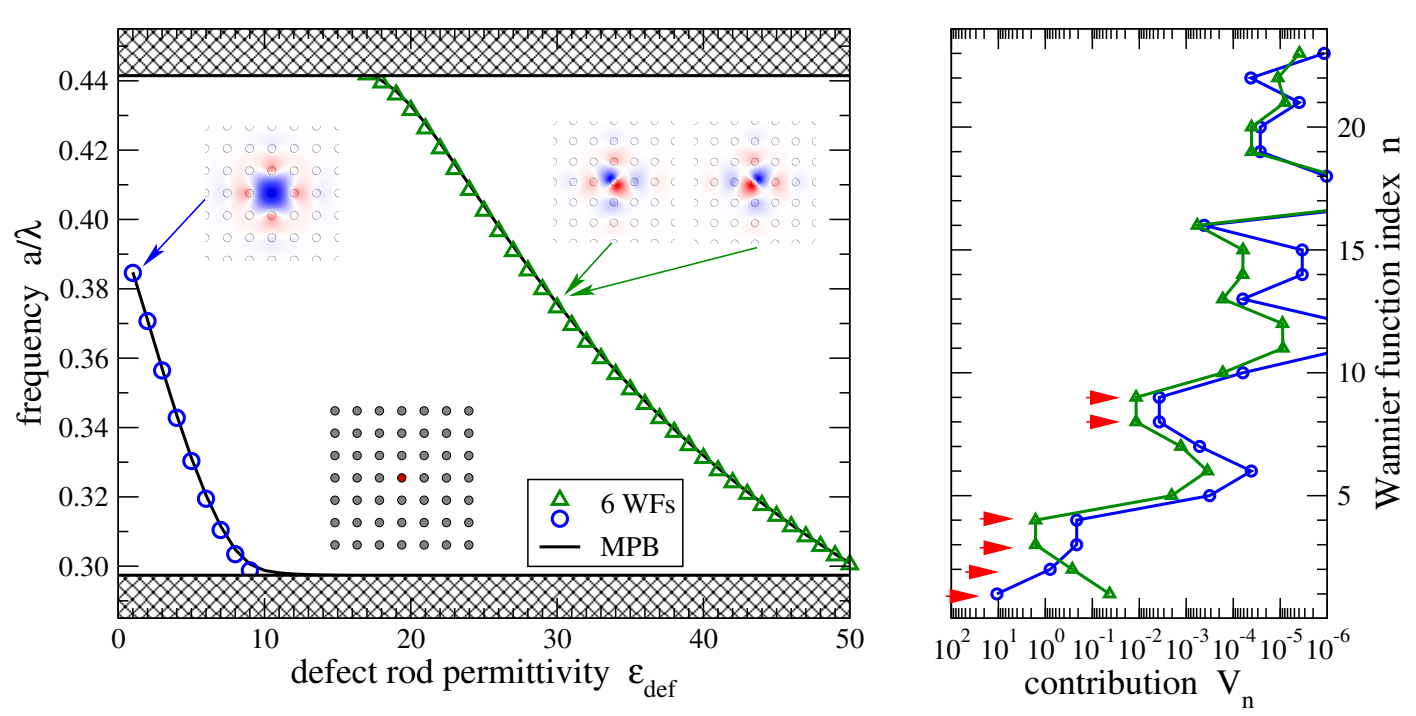

Figure 4.6.: Modes of a cavity created by varying the dielectric permittivity $\epsilon_{\text {def }}$ of a single rod. Frequencies calculated with the six most important Wannier functions (symbols) are in excellent agreement with MPB results (solid lines, $7 \times 7$ supercell, spacial resolution $a / 64$ ). The right diagram shows the contributions of individual Wannier functions to the monopole and dipole modes retrieved from a calculation using twenty-three Wannier functions. The six most important Wannier functions are indicated by red arrows.

system 1. This calculation gave converged results compared to the MPB supercell results. By analyzing the contributions $V_{n}$, Eq. (2.29) on page 28, of the individual Wannier functions to the defect modes for $\epsilon_{\text {def }}=1.0$ (monopole mode) and $\epsilon_{\text {def }}=$ 30.0 (dipole modes), we could identify the six most important Wannier functions, which contribute the most to both the monopole mode and the dipole modes. The recalculation of the cavity modes with only this reduced set of Wannier functions gives perfectly converged results for the mode frequencies. This leads us to the conclusion that it is sufficient to work with only six Wannier functions per lattice site for the cavity with variable defect rod permittivity in order to get accurate results.

We can extrapolate this conclusion to more complex defect structures composed of point defects with variable rod permittivity only. If the fields in such structures are basically superpositions of the modes of the single point defects, then we can successfully represent such fields with only six Wannier functions per lattice site. This consideration corresponds to the Linear Combination of Atomic Orbitals (LCAO) approximation for molecular orbitals in quantum chemistry or in solid-state physics.

Variable Defect Rod Radius: Instead of changing the permittivity, we can create a cavity by varying the radius of one rod in the PhC. We examine defect radii in the range of $0.18 a<r_{\text {def }}<0.50 a$. Results are plotted in Figs. 4.7 and 4.8. Similarly to 

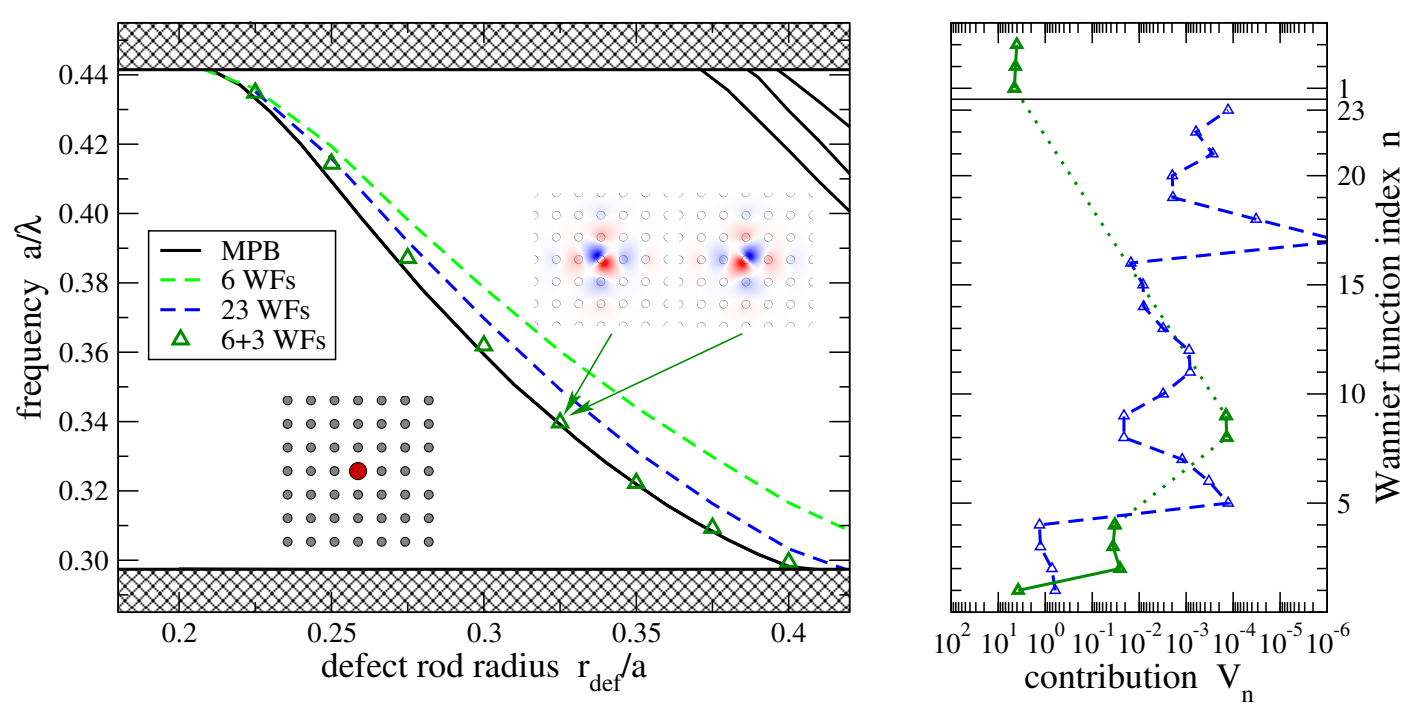

Figure 4.7.: Dipole modes of a cavity created by varying the radius $r_{\text {def }}$ of a single rod in model system 1. Wannier function results (symbols) using six or twentythree Wannier functions deviate strongly from MPB results (black solid lines, $7 \times 7$ supercell, spacial resolution $a / 64$ ). The results can be much improved by using three auxiliary Wannier functions produced for a topologically identical $\mathrm{PhC}$ with rods of radius $r=0.35 a$ to complement the six original Wannier functions.

the case when the defect rod permittivity is increased, we get a doubly-degenerate dipole mode pushed out of the upper band when increasing the radius, starting at $r_{\text {def }}=0.21 a$ (cf. Fig. 4.7). For larger defect radii $r_{\text {def }}>0.37$, also higher-order modes are evolving from the upper band, namely two quadrupole modes and one higher-order monopole mode, characterized in Fig. 4.8.

In contrast to the defects with variable permittivity, the convergence of the Wannier function results for variable defect rod radius is worse. Fig. 4.7 shows that the frequencies taking six Wannier functions per lattice site into account deviate strongly from the MPB reference solution. With twenty-three Wannier functions per lattice site, the results are improved, but are still off by up to three percent. Obviously, the number of Wannier functions per lattice site should still be increased to get accurate results. The convergence of the Wannier function expansion with the number of Wannier functions seems to be very slow.

Instead of further increasing the number of Wannier functions, we examine a different way to achieve convergence. The cavity is created by a single rod of larger radius $r_{\text {def }}$. We therefore calculate auxiliary Wannier functions for a $\mathrm{PhC}$ composed of rods with that radius $r_{\text {def }}$, which is otherwise topologically identical to the original $\mathrm{PhC}$. These auxiliary Wannier functions are supposed to reproduce the field at the largeradius defect rod better than the original Wannier functions. On the other hand, they are unnecessary and even counterproductive outside the range of the defect rod, since they are disturbing the band-gap information encoded in the original Wannier 

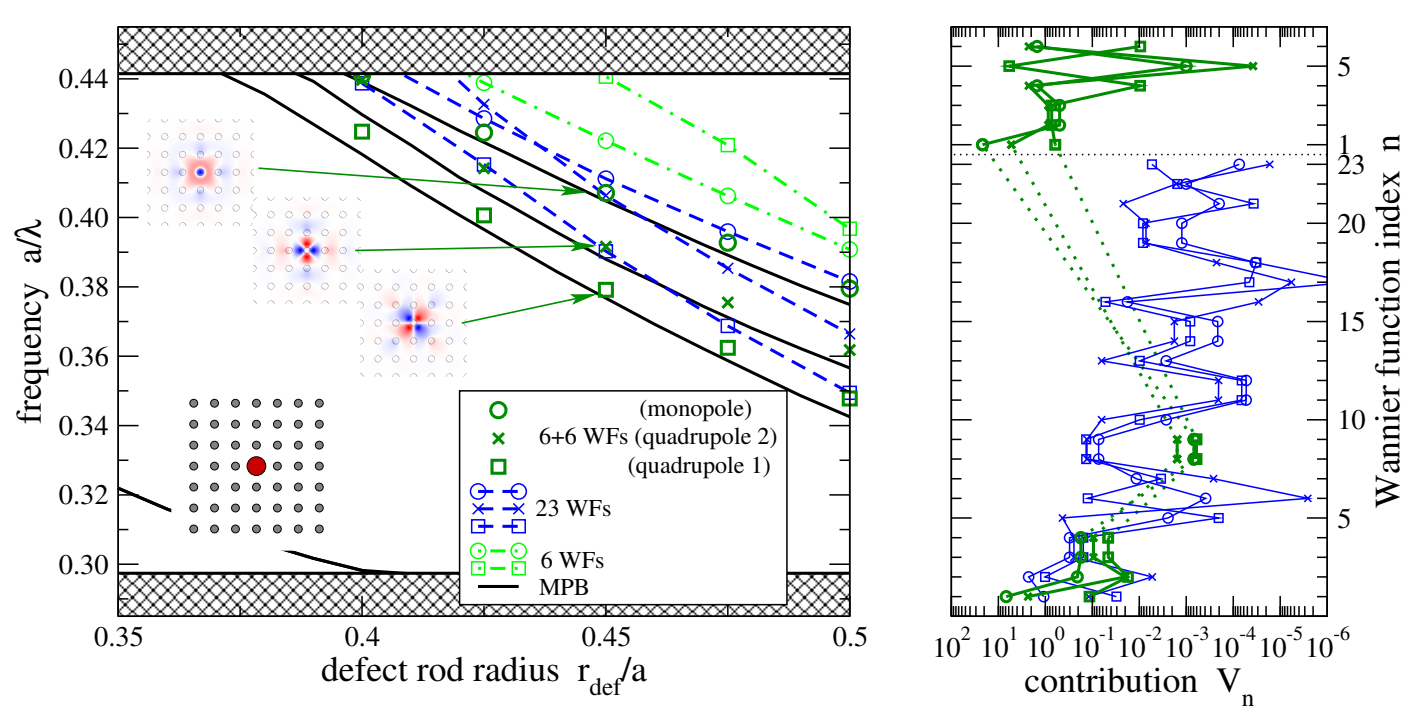

Figure 4.8.: Higher-order modes of a cavity created by varying the radius $r_{\text {def }}$ of a single rod in model system 1 . The deviations of the Wannier function results for both six and twenty-three Wannier functions from the MPB reference solution can be improved by using 6 auxiliary Wannier functions produced for a topologically identical $\mathrm{PhC}$ with rod radius $r=0.45 a$ to complement the six original Wannier functions.

functions. This can easily result in spurious modes if the auxiliary Wannier functions are used at all lattice sites of the computational domain. Thus, we use original Wannier functions at all lattice sites but only add auxiliary Wannier functions at lattice sites next to the defect rod, including the lattice site of the defect rod itself. It should also be noted that the auxiliary Wannier functions are not orthogonal to the original Wannier functions. The resulting expansion basis is no longer orthonormal, which may lead to numerical instabilities.

For the calculations with variable defect rod radius, auxiliary Wannier functions were computed for two radii, $r=0.35 a$ and $r=0.45 a$. The first three Wannier functions for $r=0.35 a$ were taken to improve results for the dipole modes, Fig. 4.7. The first six Wannier functions for $r=0.45 a$ served as auxiliary Wannier functions for the higher order modes, Fig. 4.8. Those two sets of auxiliary Wannier functions were added to the set of six original Wannier functions that was already used for the calculations with variable defect rod permittivity. Each set of auxiliary Wannier functions gives best results for a defect rod radius that corresponds to the radius the auxiliary Wannier functions were computed for. Nevertheless, the results for other defect rod radii are improved as well. The dipole mode frequencies in Fig. 4.7 deviate only up to $1.2 \%$ (maximum deviation at $r_{\text {def }}=0.25 a$ ) from the MPB results. The higher-order monopole mode frequency in Fig. 4.8 is accurate for all radii up to $1 \%$ deviation (maximum deviation at $r_{\text {def }}=0.50 a$ ). The two quadrupole modes are slightly worse but still lie within a maximum deviation of $2.5 \%$ for the second 
quadrupole mode frequency at $r_{\text {def }}=0.40 a$.

In conclusion, we can accurately compute cavity modes for single-rod defects with variable defect rod permittivity or with variable defect rod radius. We need six original Wannier functions per lattice site and, in the case of rod radius variations, up to six auxiliary Wannier functions per lattice site around the defects to get converged results.

To extend the above considerations, we compute the cavity modes of a complex cavity composed of several defects, mixing permittivity defects with radius defects. The cavity is illustrated in Fig. 4.5. The central defect is a silicon rod of radius $r_{\text {def }}=$ $0.35 a$ and is surrounded by four defects with permittivity $\epsilon_{\text {def }}=30.0$. The resulting cavity mode frequencies and mode profiles are presented in Fig. 4.9. The maximum deviation in frequency is less than $0.3 \%$ of the MPB results for all frequencies. Six original and three auxiliary Wannier functions (computed for $r=0.35 a$ ) were used for the computation.

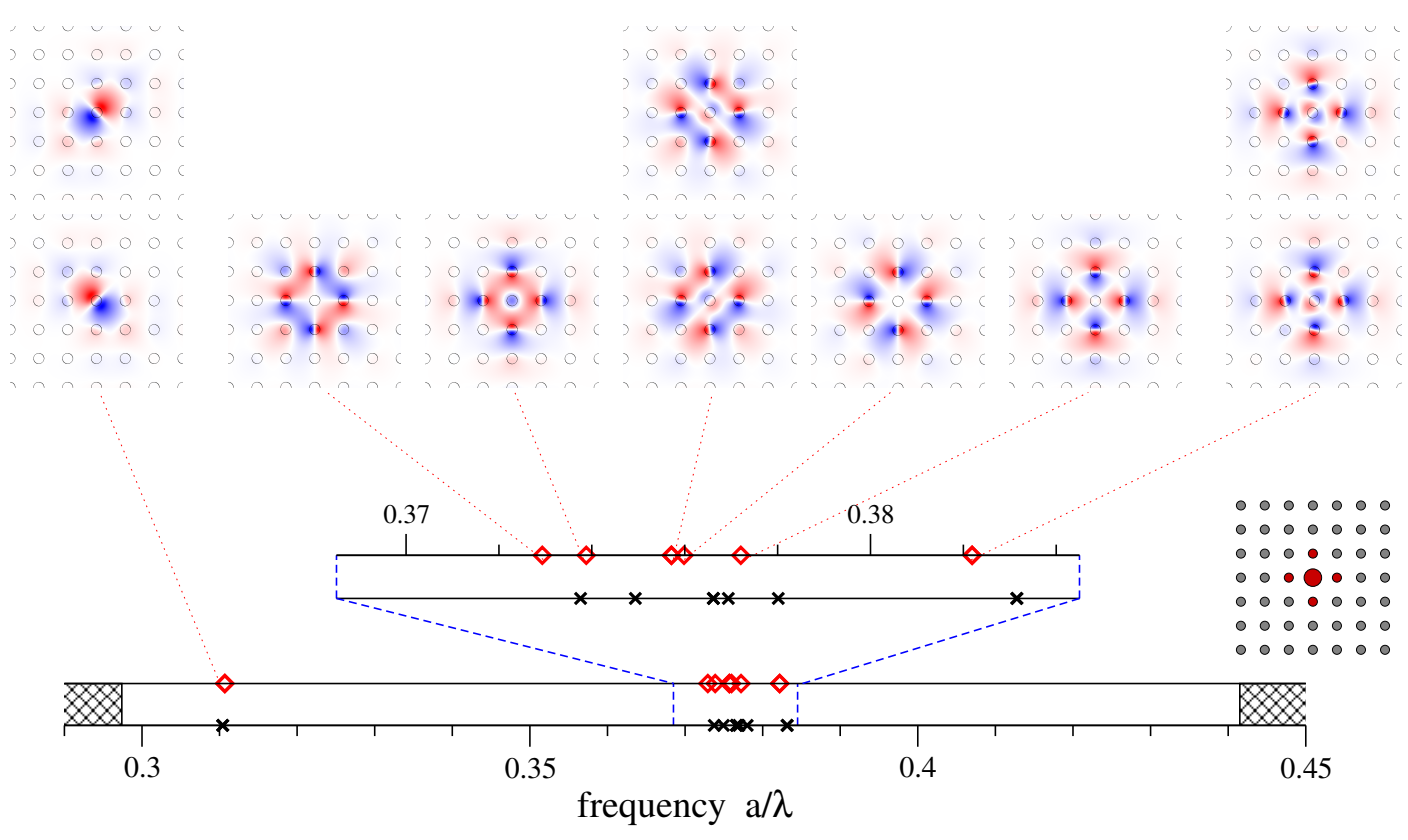

Figure 4.9.: Cavity modes of a complex cavity in model system 1 . Wannier function results are depicted by red diamonds and compared to MPB results $(7 \times 7$ supercell, spacial resolution $a / 64$ ). The frequencies agree up to a maximum deviation of $0.3 \%$.

\subsubsection{Model System 2}

In model system 2 , we restrict ourselves to defects created by single-pore infiltration as explained in section 1.2.5. We first examine a cavity created by filling a single pore with variable dielectric permittivity $\epsilon_{\text {def }}$. The resulting cavity modes and frequencies are displayed in Figs. 4.10 and 4.11. For weak defects, $\epsilon_{\text {def }}<4.0$, only a doubly- 

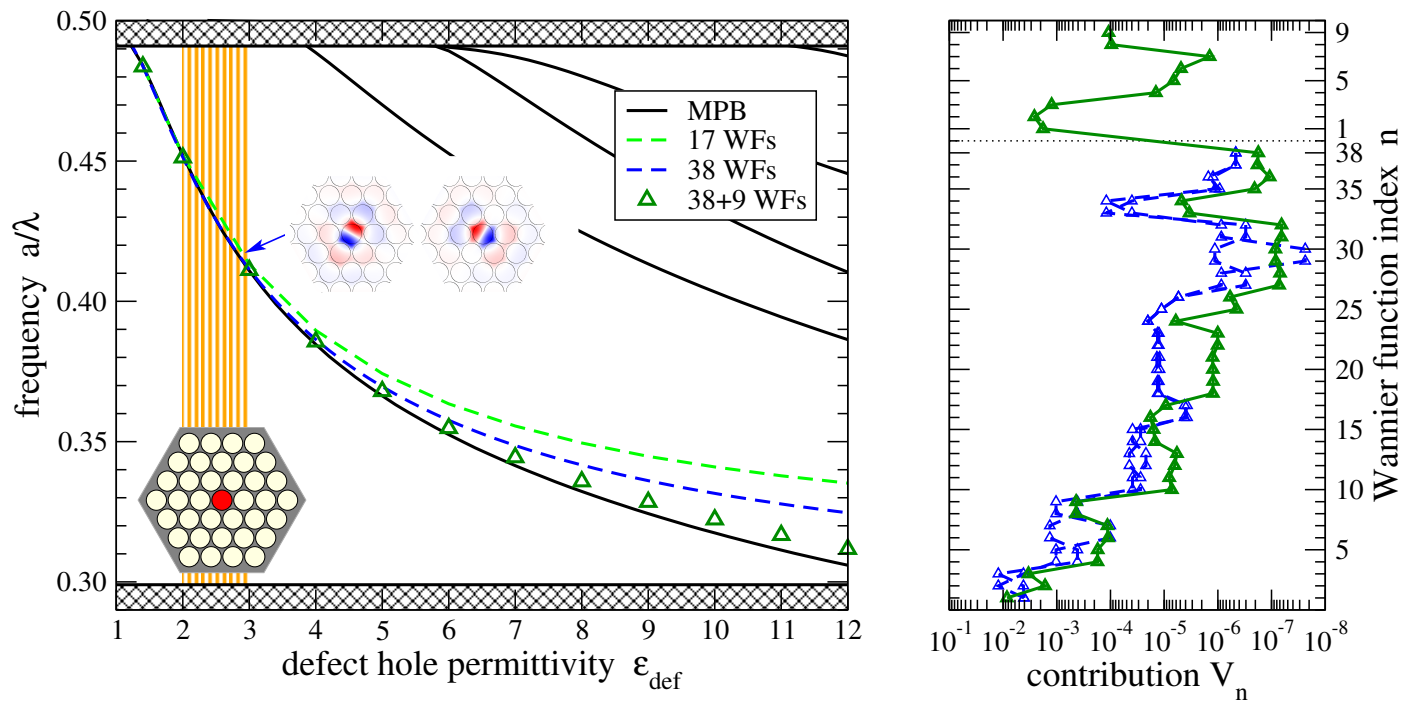

Figure 4.10.: Dipole cavity modes for a single point defect with variable dielectric permittivity $\epsilon_{\text {def }}$ in model system 2 . For weak defects (highlighted region), Wannier function results (17 or $38 \mathrm{WFs})$ agree very well with MPB calculations $(7 \times 7$ supercell, spacial resolution $a / 64)$. For stronger defects, $\epsilon_{\text {def }}>4.0$, there is a significant mismatch. It can be alleviated by adding nine auxiliary Wannier functions computed for the inverse $\mathrm{PhC}$ (silicon rods of radius $r=0.45 a$ on a triangular lattice).
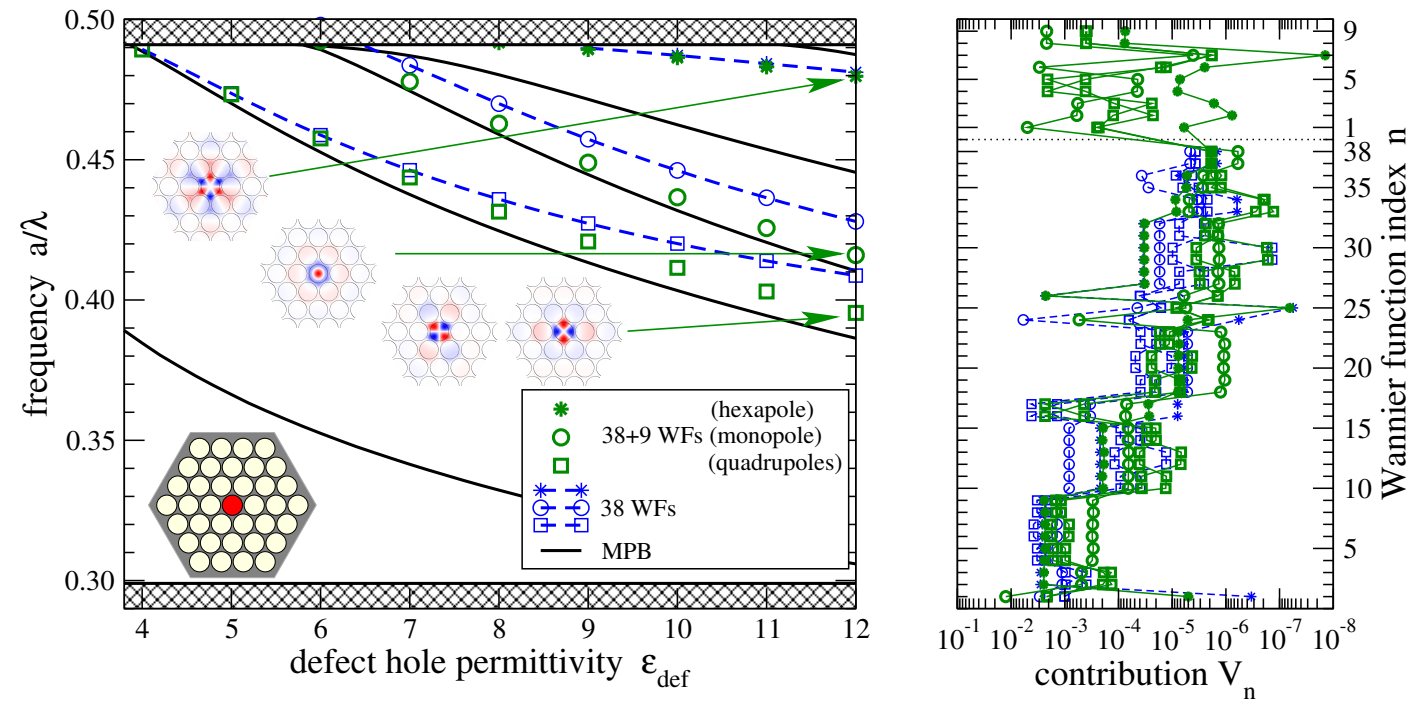

Figure 4.11.: Higher-order cavity modes for a single point defect with variable dielectric permittivity $\epsilon_{\text {def }}$ in model system 2. As in Fig. 4.10, the large mismatch between results of thirty-eight Wannier functions and MPB can be alleviated by using nine auxiliary Wannier functions. 
degenerate dipole mode is supported by the cavity. For stronger defects, $\epsilon_{\text {def }}>4.0$, higher-order modes start to evolve. Firstly, a doubly-degenerate quadrupole mode appears at the upper band edge for $\epsilon_{\text {def }} \approx 4.0$. Then a monopole and a hexapole mode are supported for increasing defect permittivity $\epsilon_{\text {def }}$.

The dipole mode for weak defects is well represented with thirty-eight or seventeen Wannier functions, cf. Fig. 4.10. The seventeen Wannier functions have been selected out of the thirty-eight Wannier functions by taking the ones with the largest contribution $V_{n}$ to the dipole mode for $\epsilon_{\text {def }}=12.0$ according to (2.29). It is apparent though from Figs. 4.10 and 4.11 that for stronger defects, such as non-etched pores $\left(\epsilon_{\text {def }}=12.0\right)$, both the dipole mode frequency and the higher order mode frequencies deviate significantly from the MPB reference solution even when taking into account all thirty-eight Wannier functions available. It would be necessary to use even more Wannier functions.

One reason for the slow convergence with number of Wannier functions may be the strict boundary conditions imposed on $\mathrm{H}$-polarized fields. The Wannier functions show significant kinks at discontinuities of the dielectric permittivity in the host $\mathrm{PhC}$. Such kinks may be counterproductive when a cavity mode for a non-etched pore (i.e., continuous dielectric permittivity at the central pore) has to be modeled.

Instead of increasing the number of Wannier functions, we use the same strategy as for the radius defect cavity calculations in model system 1 in order to get better converged results. We introduce auxiliary Wannier functions to complement the original Wannier function basis by appropriate additional field components that are supposed to better reproduce fields at infiltrated-pore defects or non-etched pores. In the present case, the $H$-polarization Wannier functions for the inverse system, i.e., a triangular lattice of silicon rods of radius $r=0.45 a$, may be a good choice as a set of auxiliary Wannier functions. Therefore, we have computed the first nine Wannier functions for the inverse system, depicted in Fig. 4.4. It is apparent from Figs. 4.10 and 4.11 that the use of those nine functions at the defect lattice site and the immediate neighbors together with the thirty-eight original Wannier functions at all lattice sites of the computational domain leads to much more accurate results for strong defects, both for the dipole modes and the higher-order modes, except for the hexapole mode, which is still not represented very well by the extended Wannier function basis.

The use of auxiliary Wannier functions is still under investigation and has to be further tested. For the cases discussed so far, both for model system 1 and 2, the results are very promising. Nevertheless, for the circuit design in chapter 5, we restrict ourselves to the case of weak defects for model system 2, for which the Wannier function expansion works fine without using auxiliary Wannier functions.

Finally, we calculate the cavity modes of a more complex cavity that consists of a ring of six pores filled with a low-index material with dielectric permittivity $\epsilon_{\text {def }}=2.89$. The resulting mode profiles and frequencies are depicted in Fig. 4.12. The frequencies are in perfect agreement with MPB reference results. 


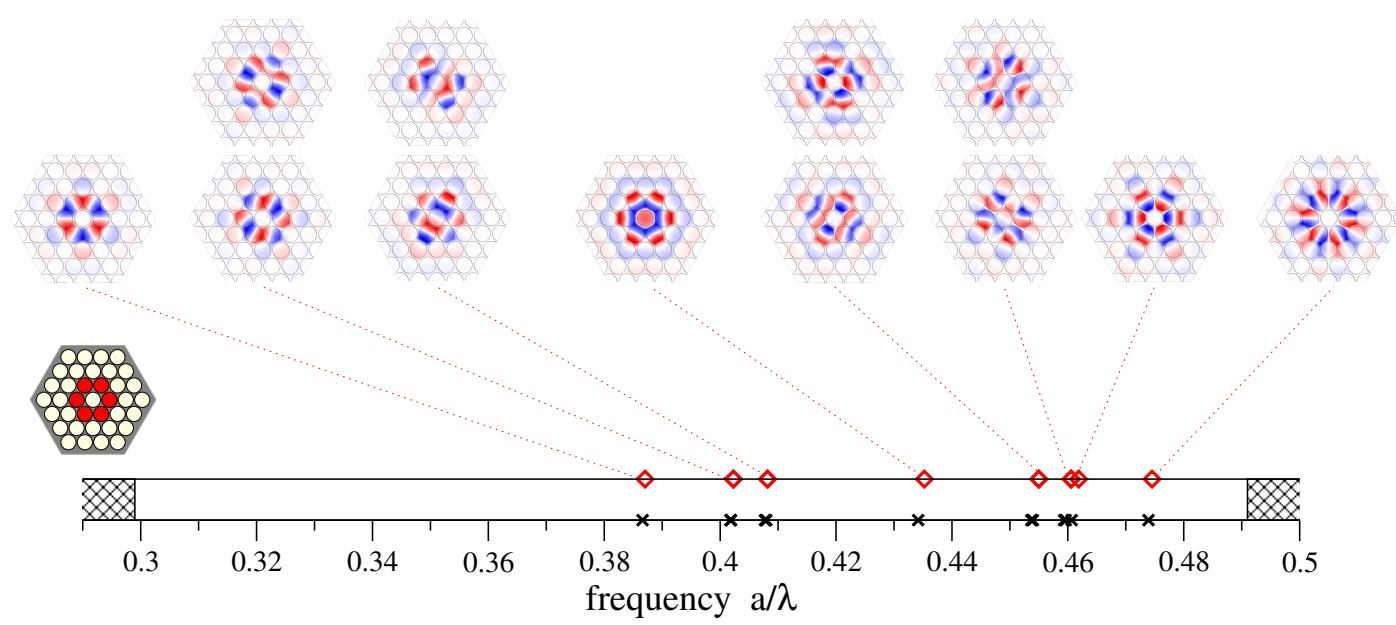

Figure 4.12.: Complex cavity (ring) in model system 2. Six pores are filled with lowindex material with $\epsilon_{\text {def }}=2.89$. The relative difference between Wannier function results (red diamonds) and converged MPB results (black crosses, supercell $9 \times 9$, spacial resolution $a / 64$ ) is less than 0.5 percent.

\subsection{Waveguide Dispersion}

Next, we discuss calculations of waveguide dispersions for different line defects in model systems 1 and 2 and compare them to MPB reference calculations. The line defects are composed of the same point defects already discussed in the previous section about cavity modes. Many conclusions about the necessary number of Wannier functions and the use of auxiliary Wannier functions can be adopted here.

\subsubsection{Model System 1}

In model system 1, we begin with the case of two line defects created by removing a row of rods or changing the dielectric permittivity of the rods to $\epsilon_{\text {def }}=30.0$, respectively. Such line defects are directly related to the cavity class of a single rod with variable dielectric permittivity discussed in Fig. 4.6, for which we got excellent agreement with MPB by using only the six most important Wannier functions per lattice site. Consequently, Fig. 4.13 shows that the waveguide dispersion of such line defects is accurately reproduced using only six Wannier functions.

Similarly, we construct a line defect of one row of rods with radius $r_{\text {def }}=0.35 a$, corresponding to the cavity class supporting a degenerate dipole mode, discussed in Fig. 4.7. As for the cavity modes, the MPB reference waveguide dispersion, plotted in Fig. 4.14, is reproduced only qualitatively by using six Wannier functions. It can be improved by taking twenty-three Wannier functions into account, but there is still a certain offset to the reference solution. Nevertheless, to get quantitative agreement with MPB, we can use three auxiliary Wannier functions computed for a topologically 


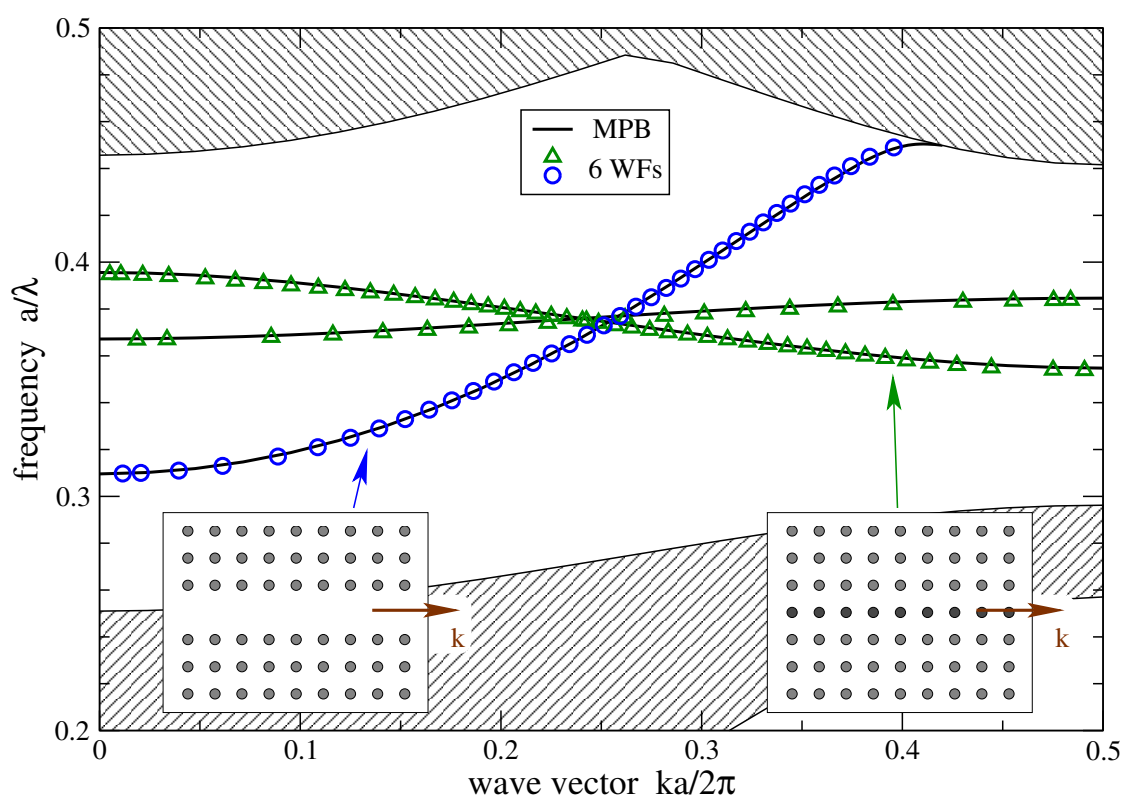

Figure 4.13.: Waveguide dispersion of a line defect of missing rods (blue circles) and rods with $\epsilon_{\mathrm{def}}=30.0$ (green triangles) in model system 1. In both cases, the calculated results using six Wannier functions agree perfectly with MPB results (supercell size $9 a$, spacial resolution $a / 64)$.

identical $\mathrm{PhC}$ with rods of radius $r=0.35 a$ in addition to the six original Wannier functions. These results are consistent with the ones obtained in Fig. 4.7.

The third class we consider is a line defect created by changing the radius of a row of rods to $r_{\text {def }}=0.45 a$. This waveguide corresponds to the cavity class discussed in Fig. 4.8, which supports several higher-order modes. Accordingly, the waveguide dispersion in Fig. 4.15 shows a richer spectrum of guided modes. This spectrum can hardly be reproduced by using only six Wannier functions per lattice site. Again, the use of twenty-three Wannier functions improves the results significantly. Almost quantitative agreement to the MPB reference solution can be obtained by applying auxiliary Wannier functions computed for a topologically identical $\mathrm{PhC}$ with rods of radius $r=0.45 a$ in addition to the six original Wannier functions. However, the use of auxiliary Wannier functions leads to the appearance of a spurious mode in the waveguide dispersion for this particular case. The spurious mode arises in Fig. 4.15 as a steep band with quadratic dispersion at the Brillouin zone edge. For the calculations, we used five out of the six auxiliary Wannier functions for $r=0.45 a$ in Fig. 4.3(b) and applied them only at the defect sites. We left out the first, monopolelike Wannier function for $r=0.45 a$, since it lead to even more spurious modes appearing at different wave vectors in the dispersion.

The origin of spurious modes can be clearly attributed to the auxiliary Wannier functions, which introduce field components that are in conflict with the band gap 


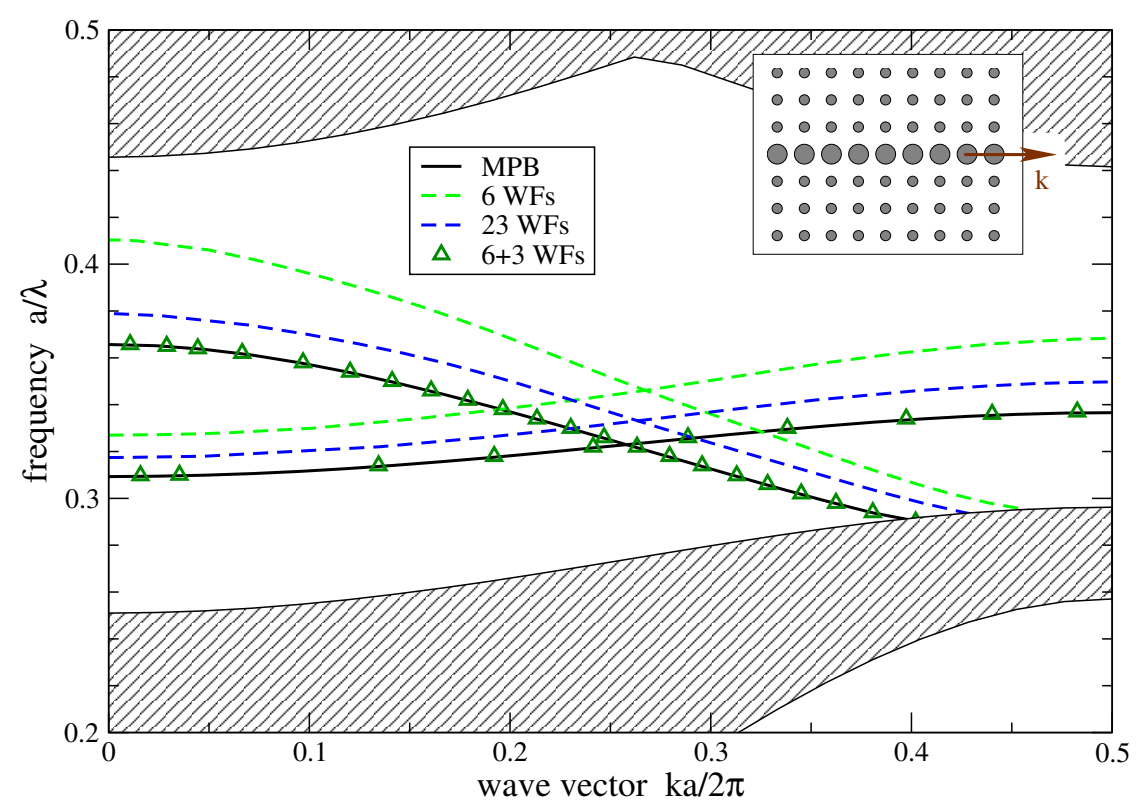

Figure 4.14.: Waveguide dispersion of a line defect of rods with radius $r=0.35 a$ in model system 1 . While the results using six as well as twenty-three Wannier functions (dashed lines) reproduce the MPB reference solution (solid lines, supercell size $9 a$, spacial resolution $a / 64$ ) only qualitatively, the results become quantitatively correct when we use six original Wannier functions plus three auxiliary Wannier functions computed for a topologically identical $\mathrm{PhC}$ with rods of radius $r=0.35 a$, see Fig. 4.3(a).

of the underlying PhC. Since the Wannier function basis consists of only a small number of very specialized functions, the projection of the exact, infinite problem to the finite Wannier basis must be such that the number and characteristics of solutions is retained. This prerequisite seems to be broken in some cases when we use auxiliary Wannier functions. On the other hand, the non-orthogonality of the basis may lead to numerical instabilities. Both statements may be the reason for the appearance of spurious modes. This problem is still under investigation. Note, however, that the present case, radius defects with $r_{\text {def }}=0.45 a$, is a very strong deviation from the underlying periodic structure. Weaker defects, for example the ones with $r_{\text {def }}=0.35 a$ discussed above, can be handled more successfully with auxiliary Wannier functions without getting spurious modes.

Finally, we compute the waveguide dispersion of a more complicated line defect created by changing three adjacent rows of the $\mathrm{PhC}$. In the central row, we put rods with radius $r_{\text {def }}=0.35 a$, while in the other two rows, we remove the rods or replace them with rods of different permittivity $\epsilon_{\text {def }}=30.0$, respectively. The dispersion of this asymmetric waveguide is plotted in Fig. 4.16. It shows excellent agreement between Wannier function results and MPB reference calculations. We 


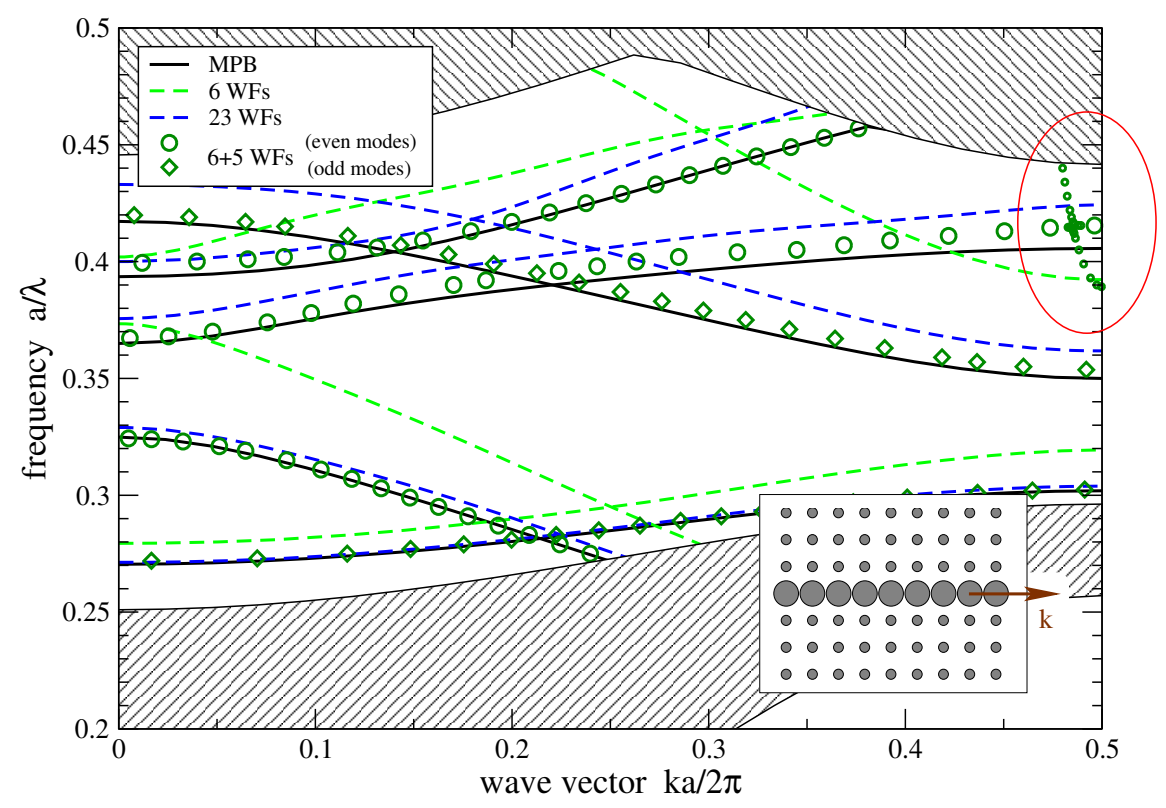

Figure 4.15.: Waveguide dispersion of a line defect of rods with radius $r=0.45 a$ in model system 1 . The MPB reference solution (solid lines, supercell size $9 a$, spacial resolution $a / 64$ ) can not be reproduced by using six Wannier functions, and the results for twenty-three Wannier functions deviate significantly. Using five auxiliary Wannier functions computed for a topologically identical $\mathrm{PhC}$ with rods of radius $r=0.45 a$ (WFs 2-6 in Fig. 4.3(b)) improves the quantitative agreement to MPB. However, a spurious mode appears, highlighted by the red ellipse in the top-right corner of the spectrum.

used six original Wannier functions plus three auxiliary Wannier functions for a $\mathrm{PhC}$ with rods of radius $r=0.35 a$.

\subsubsection{Model System 2}

In model system 2, we investigate three different waveguides in the framework of single-pore infiltration. The simplest one is formed by a row of pores filled with a low-index material of dielectric permittivity $\epsilon_{\text {def }}=2.89$. The point defects in this waveguide correspond to the cavity discussed in Fig. 4.10 in the regime of weak defects. A single point defect of this type supports two dipole modes only, therefore, the waveguide dispersion, depicted in Fig. 4.17, shows two bands crossing each other. The results calculated using the first twenty-six Wannier functions of model system 2 are in very good agreement with the MPB reference solution.

Next, we discuss a one-row waveguide formed by stronger defects, in which the pores are not etched or, equivalently, are filled with silicon $\left(\epsilon_{\text {def }}=12.0\right)$. Fig. 4.18 shows that this waveguide is strongly multi-mode over wide frequency ranges, as 


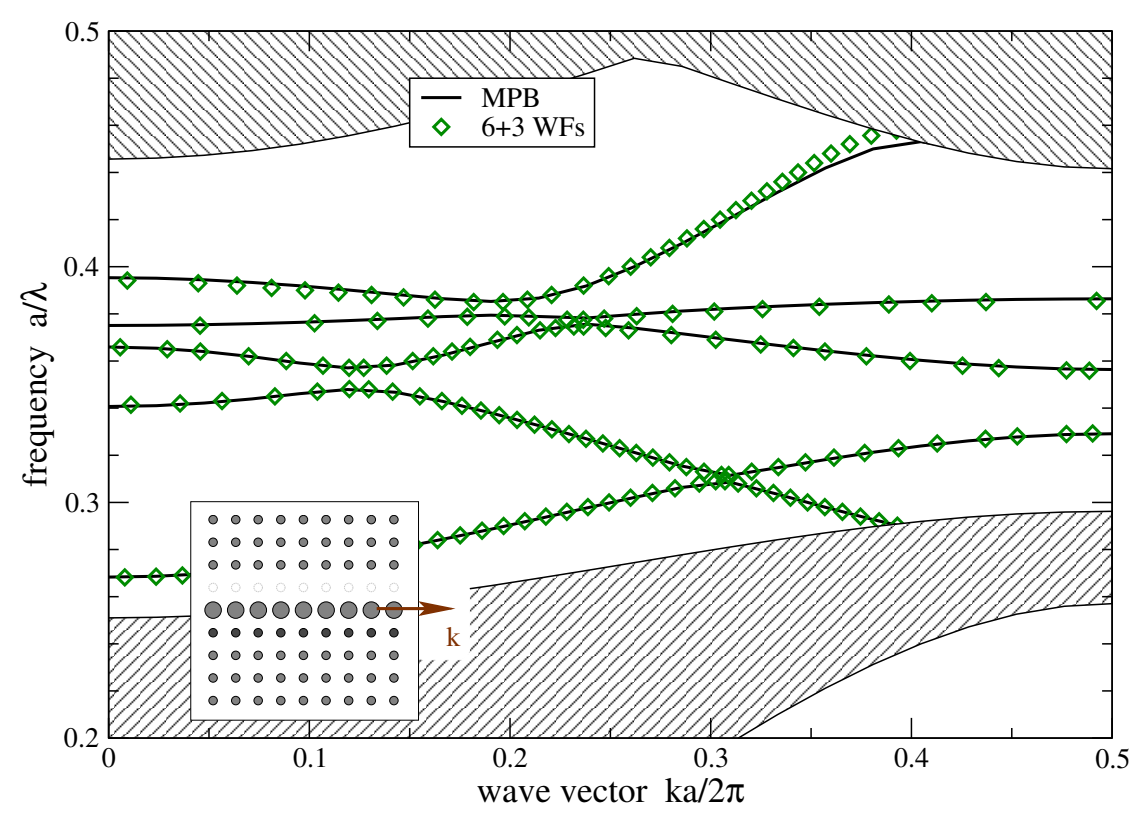

Figure 4.16.: Complex waveguide in model system 1 consisting of one row of missing rods, one row of rods with radius $r_{\text {def }}=0.35 a$ and one row of rods with permittivity $\epsilon_{\text {def }}=30.0$. Results calculated with six original Wannier functions plus three auxiliary Wannier functions for a PhC with $r=0.35 a$ (cf. Fig. 4.3(a)) are in excellent agreement with MPB calculations (supercell size 11a, spacial resolution $a / 64$ ).

the single non-etched pore supports seven cavity modes (cf. Fig. 4.11), which are all reflected in the waveguide dispersion. It is apparent from Fig. 4.18 that it is not possible to get quantitatively correct results with the full set of thirty-eight original Wannier functions for model system 2. Some bands can be qualitatively identified, but they are significantly shifted to higher frequencies. Again, we examine the results when taking nine auxiliary Wannier functions for the complementary system of rods into account. Indeed, the results get quantitatively better. However, the deviation, especially for higher bands, is still large.

We complete the discussion of waveguide dispersions by an example for a more complex waveguide in model system 2 , which is formed by three adjacent rows of filled pores. The pores in the two outer rows are filled with a material of $\epsilon_{\text {def }}=2.89$, while the central pores are filled with a material of $\epsilon_{\text {def }}=2.40$. The dispersion of this waveguide is depicted in Fig. 4.19. This waveguide only consists of pores filled with low-index material. Consequently, using twenty-six Wannier functions, we get good agreement as compared to the reference solution. 


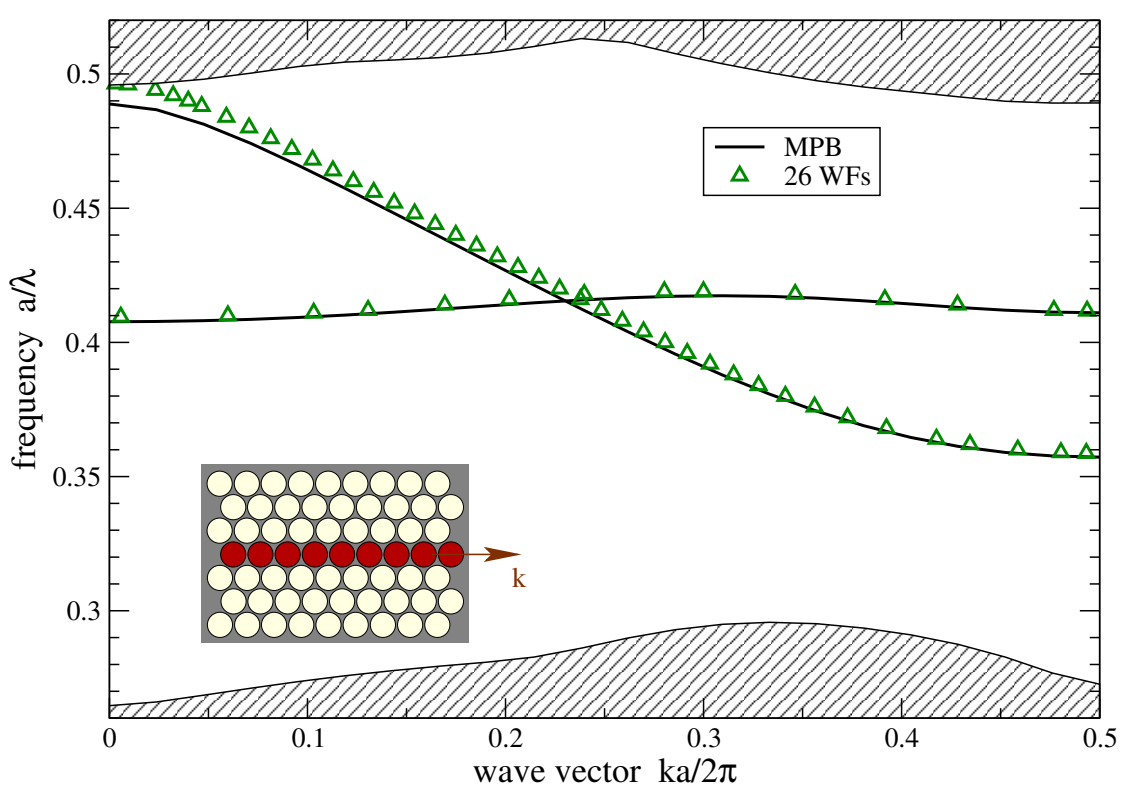

Figure 4.17.: Low-index waveguide in model system 2. A row of holes is filled with material of $\epsilon_{\mathrm{def}}=2.89$. The Wannier function results computed with the first twentysix Wannier functions agree well with MPB calculations (super cell extent 9, spatial resolution $a / 96$ ). The deviation exceeds $1 \%$ only near the upper band edge.

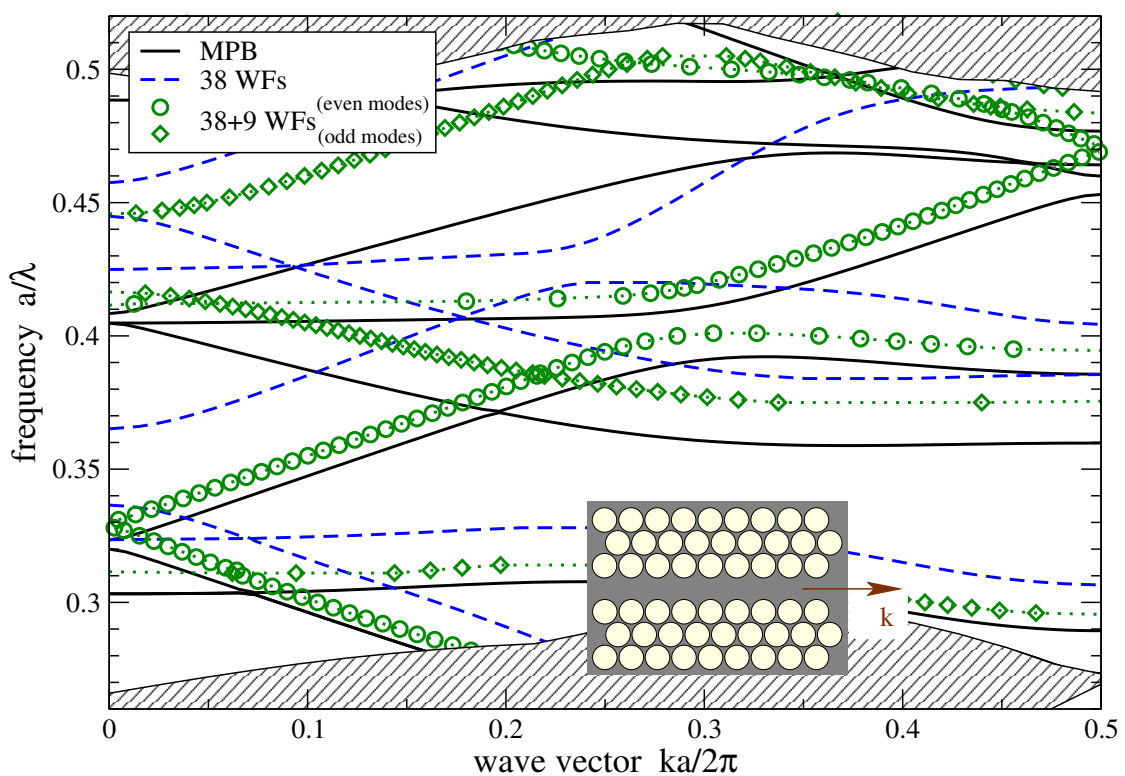

Figure 4.18.: Waveguide formed by a row of non-etched pores in model system 2 . Similar to the cavity mode calculations for a single non-etched pore $\left(\epsilon_{\mathrm{def}}=12.0\right)$, the calculations using thirty-eight Wannier functions significantly differ from the reference calculations using MPB (super cell extent 9, resolution a/96). The use of nine auxiliary Wannier functions for the complementary rod system relieves the deviation a bit. 


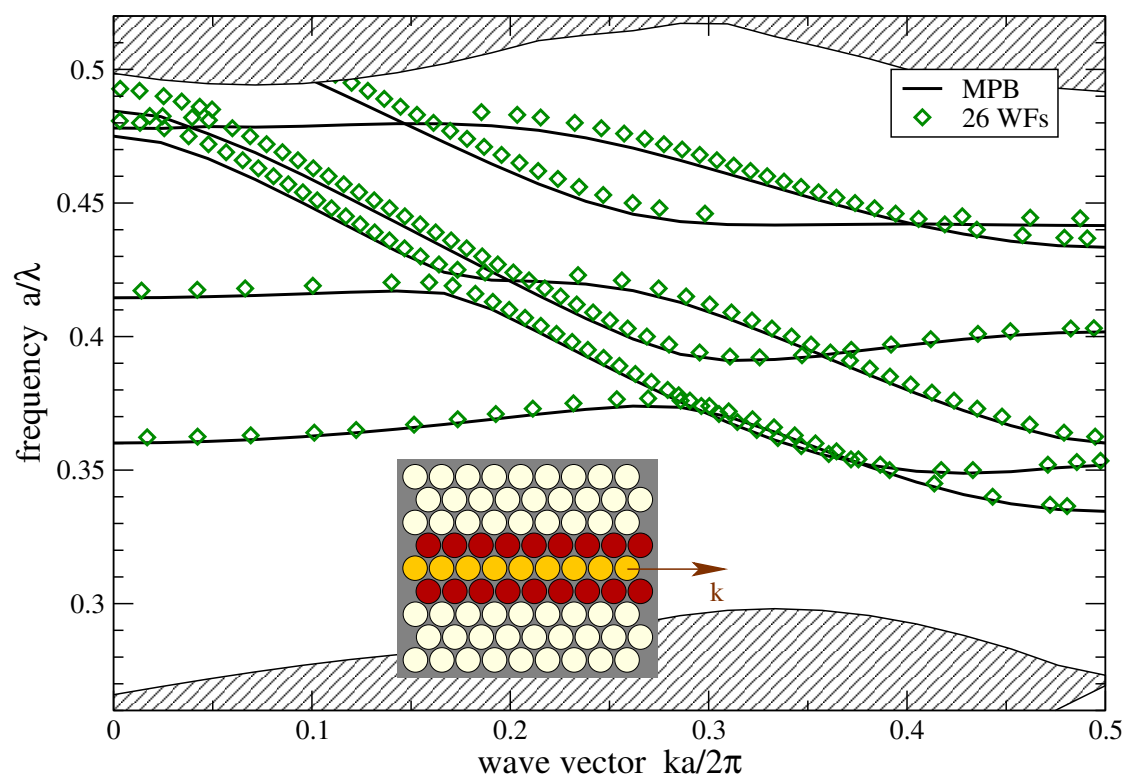

Figure 4.19.: Complex waveguide formed by three adjacent rows of filled pores in model system 2. The outer two rows are filled with a material of $\epsilon_{\text {def }}=2.89$, the central row with a material of $\epsilon_{\mathrm{def}}=2.40$. Results using twenty-six Wannier functions agree well with the MPB reference solutions (super cell extent 11, spacial resolution $a / 64)$.

\subsection{Waveguide as a Functional Element}

The S-matrix of a waveguide of finite length $L$ has a very simple form. It is reflectionless and contains only exponentials of the guided-modes' phase shifts over the length of the waveguide,

$$
S_{\mathrm{wg}}=\left(\begin{array}{cc}
0 & G \\
G^{T} & 0
\end{array}\right) \quad \text { with } \quad G=\left(\begin{array}{lll}
\ddots & & 0 \\
& \exp \left(\mathrm{i} k_{\ell} L\right) & \\
0 & & \ddots
\end{array}\right) .
$$

As a check, we can either calculate this S-matrix using the waveguide dispersion, or we can treat the waveguide as an ordinary two-port FE and compute the Smatrix for this FE. Fig. 4.20 shows the resulting comparison. By construction, the S-matrix derived from dispersion data, (4.1), has zero reflectance and a phase of the transmission elements $\phi_{T}(\omega)=k(\omega) \cdot L$. Therefore, we plot the reflectance of the FE-computed S-matrix, which should physically be zero, and the transmission phase deviation $\Delta \phi$ of the FE-computed S-matrix with respect to the phase $\phi_{T}(\omega)$ from dispersion data. These values can give an indication for the necessary distance between two ports of an FE. For short distances, the direct coupling between the two ports may lead to wrong results for the S-matrix of an FE. It is apparent from 

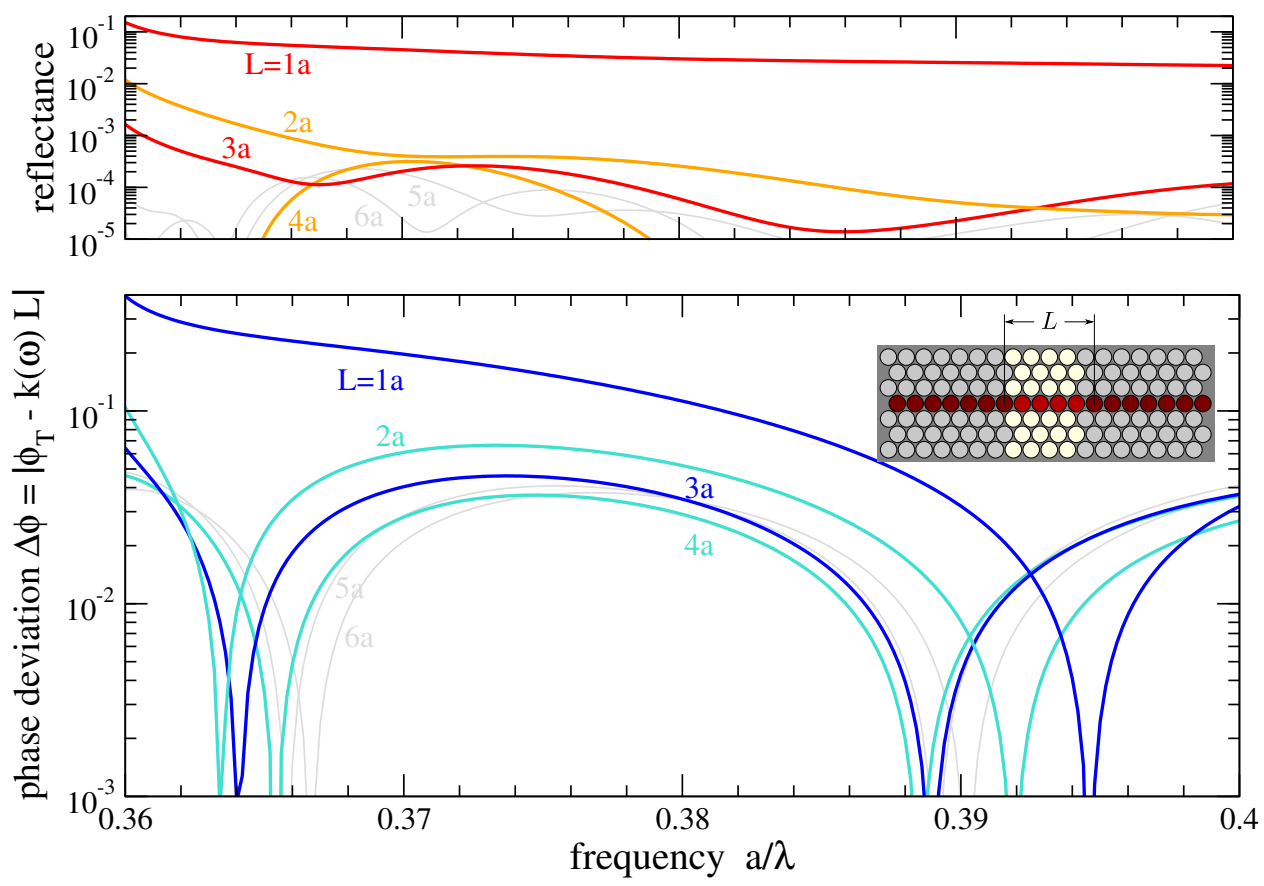

Figure 4.20.: Finite waveguide section of a single-mode waveguide $\left(\epsilon_{\mathrm{def}}=2.89\right)$ in model system 2 treated as a functional element. The reflectance, which should physically be zero, and the phase deviation $\Delta \phi$ from dispersion-derived data are plotted against frequency. For lengths $L \geq 3 a$, the numerically calculated reflectance is less than $10^{-3}$ and $\Delta \phi$ is well below $10^{-1}$. Thirty-eight Wannier functions were used.

Fig. 4.20 that a distance of $L \geq 3$ is sufficient to get reasonably low direct coupling between ports in this particular case. The reflectance does not exceed $10^{-3}$ and the phase deviation is well below 0.1 .

We may conclude that the distance between ports of FEs may be chosen quite small. Note, however, that this can not be generalized to arbitrary kinds of FE.

\subsection{Double Bend}

According to section 3.2.1, we reduce the individual S-matrices of basic FEs to the blocks relating propagating guided modes only. S-matrix entries for evanescent guided modes are ignored. For this case, the S-matrix combination to obtain the full S-matrix of a large-scale circuit becomes numerically extremely simple, since the matrices involved are very small (typically $2 \times 2$ or $4 \times 4$ ). This approximation requires that the field components that evanescently decay away from the individual FEs have negligible overlap between the FEs, and only the propagating guided modes in the waveguides between the FEs contribute to the coupling of the FEs. That means, we 


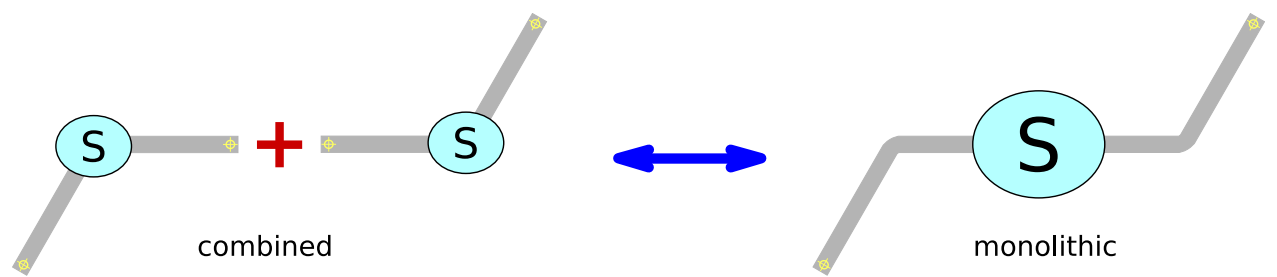

Figure 4.21.: Comparison of combined and monolithic S-matrices taking the double bend as an example. The validity of the reduced S-matrix approximation is checked, since ignoring the S-matrix entries corresponding to evanescent guided modes may lead to deviations of the combined from the monolithic S-matrix, especially for small distances of individual FEs.

have to check how far away from each other FEs must be placed to ensure that the reduced S-matrix approximation is valid. Therefore, for mid-size devices that can be divided into basic FEs, we compare the composed S-matrix, i.e., the full S-matrix that results by combining individual S-matrices of basic FEs, with the monolithic S-matrix, which results from treating the whole device as one block.

To be specific, we look at simple double bends depicted in Fig. 4.22. These devices are composed of two opposite bends that are concatenated with a certain distance $L$ between their centers. We first consider the double bend in model system 1 , Fig. 4.22(a), for which the transmittance and phase spectra are displayed in Fig. 4.23 for four different distances $L=a, 3 a, 5 a$ and $7 a$. Except for the $L=a$ case, the transmittance extracted from the combined S-matrix agrees well with the monolithic S-matrix calculation. The same is true for the transmission and reflection phases. Some small shifts in frequency of the transmittance and the reflection phase are visible for $L=3 a$ and $L=5 a$. However, we can conclude that the double bend with a quite short distance of $L=3 a$ can already be accurately handled by a reduced S-matrix combination. The evanescent coupling between the two single bends seems to be small compared to the propagating parts, even at such short distances.

Note that we use negative waveguide lengths between the two single bends if the distance $L$ is shorter than the distance that results when two single bends are connected without any space between them. Negative waveguide lengths correspond to negative phase shifts that eliminate the phase shifts already encoded in the S-matrices of the single bends due to the short waveguide stubs at their ports.

Next, we turn to the double bend in model system 2, Fig. 4.22(b). The spectra for this system are plotted in Fig. 4.24. Again, the overall agreement between combined and monolithic S-matrix data is very good, even for short distances. There are some deviations for $L=a$ and, to a smaller extent, for $L=3 a$. The agreement for $L=5 a$ and $7 a$ is excellent, though. We conclude that a distance of $L=5 a$ is sufficient for a good accuracy of combined S-matrix computations in this particular case.

Note that, in general, the necessary distance between two FEs may strongly depend on the resonant behavior of the FEs. In the above cases, the guidance in the two bends 


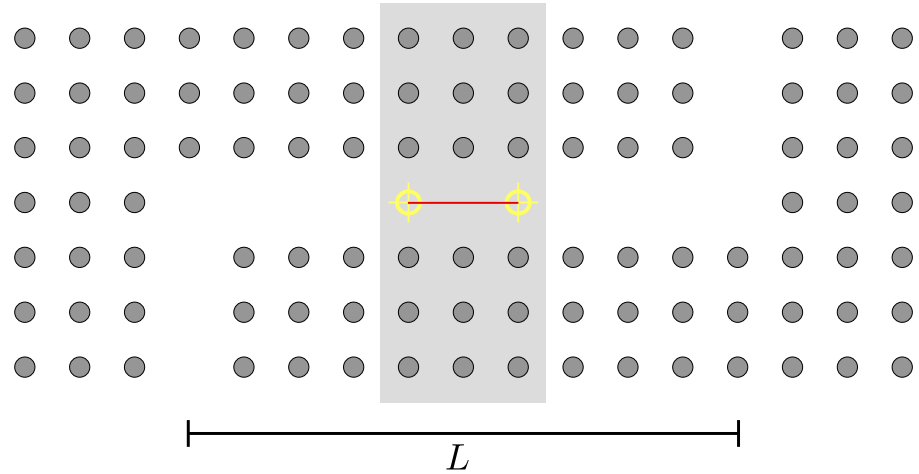

(a) in model system 1

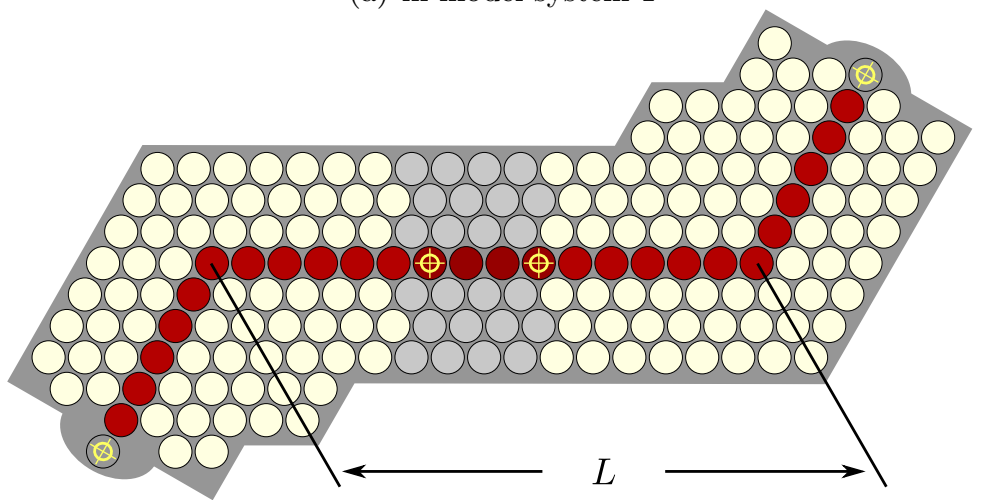

(b) in model system 2

Figure 4.22.: Double bends in the two model systems. The S-matrix of the entire double bend can either be calculated by combining S-matrices of single bends or by directly computing the S-matrix of a monolithic double bend. When combining S-matrices, we can simulate shorter double bends by assuming that the waveguide in between the two single bends (shaded region) has a negative length, which is equivalent with a negative phase shift.

is non-resonant, so the effect of the propagating field components is dominating the FE behavior. The evanescent components may play a much bigger role for resonantlycoupled FEs, for which we may need larger distances of FEs in a circuit to get accurate results for combined S-matrices.

\subsection{Simple Mach-Zehnder Interferometer}

As another simple check for the S-matrix approach, we consider a slightly more complicated composed device that can still be computed en bloc, such as a simple Mach-Zehnder interferometer (MZI) in model system 1, depicted in Fig. 4.25. The circuit can be decomposed into two T-splitters and four bends. Since the distance between the T-splitter and the bends is very small, the connections between those 

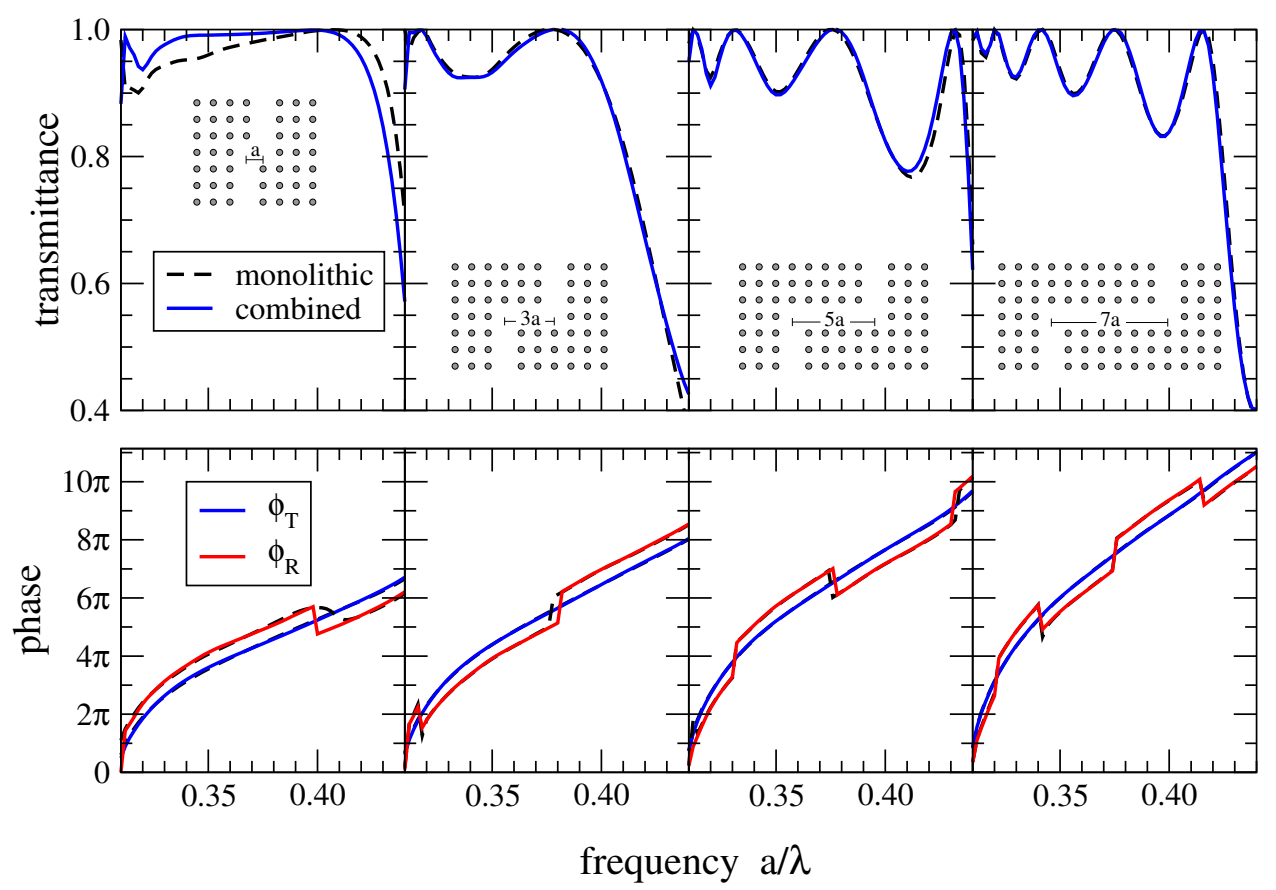

Figure 4.23.: Double bend in model system 1: Transmittance and transmission/reflection phase spectra for different distances of the single bends, $L=$ $a, 3 a, 5 a, 7 a$, are plotted. Results computed by combining S-matrices of two opposite bends (solid lines in color) are compared to results for the S-matrix of one monolithic double-bend. It is apparent that even for small distances, $L=3 a$, the results are in good agreement. Both cases were calculated using six Wannier functions, according to Fig. 4.6. The S-matrix combination for the smaller lengths $L=a, 3 a, 5 a$ was done using negative lengths for the connecting waveguide, which is equivalent to negative phase shifts.

FEs are treated as waveguides with negative lengths. This trick is the same as for the double bends discussed in the previous section.

Similar to the double bend, we compare the combined and the monolithic circuit S-matrix of the MZI. Both are computed using the six Wannier functions in Fig. 4.2. The corresponding transmittance and transmission/reflection phases are plotted in Fig. 4.26. We find that the agreement between the combined and the monolithic S-matrix is very good. The Fabry-Perot dips are well reproduced in both their depth and their frequency. Individual peaks and dips seem to be slightly shifted in frequency. However, this shift is well below $1 \%$.

The simple MZI is a testbed for large-scale circuits, which will be discussed in the following chapter. The circuits discussed there are too large to be handled monolithically. 

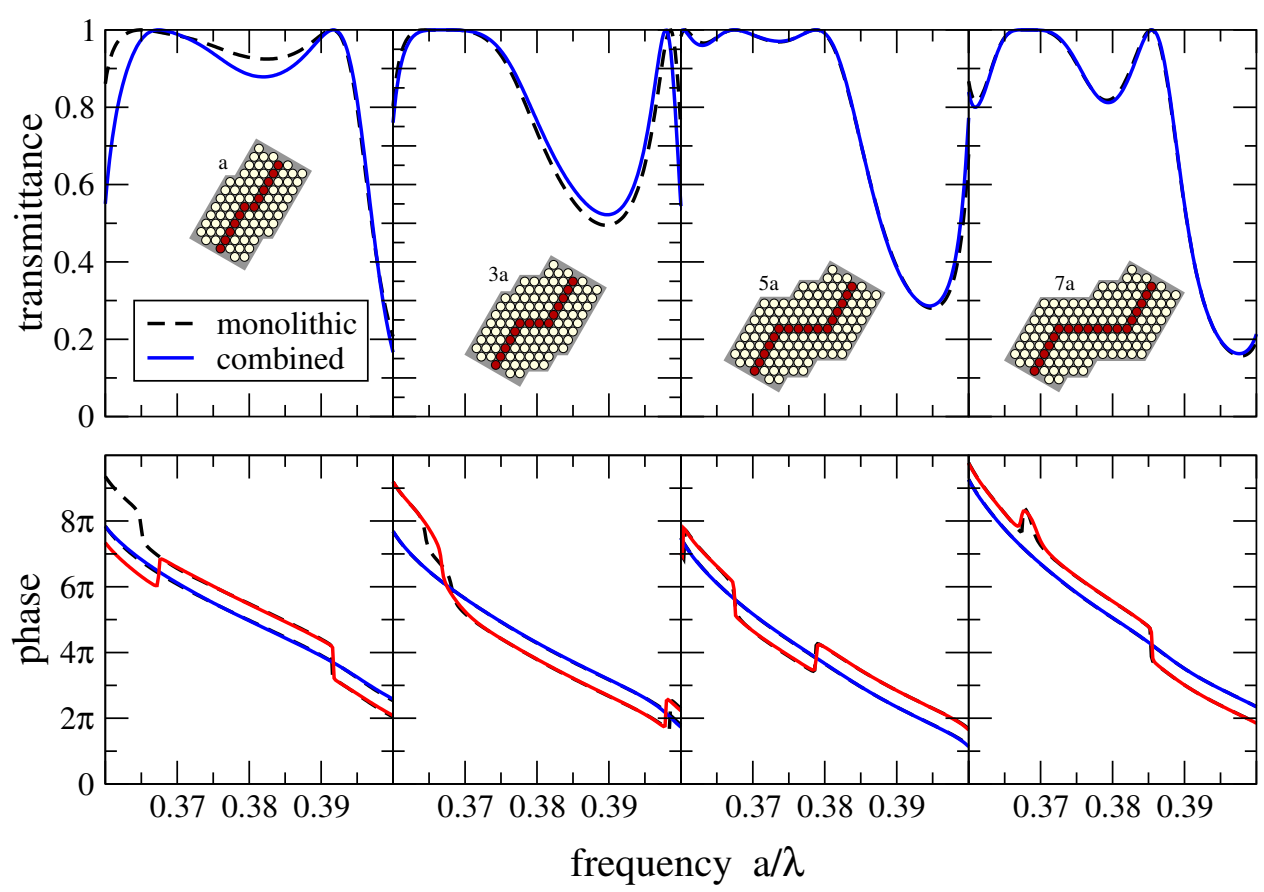

Figure 4.24.: Double bend in model system 2: Transmittance and transmission/reflection phase spectra for different distances of the single bends, $L=$ $a, 3 a, 5 a, 7 a$. Results computed by combining S-matrices of two opposite bends (solid lines in color) are compared to results for the S-matrix of one monolithic doublebend. While there are some deviations for $L=a$ and $3 a$, the agreement for $L=5 a$ and $L=7 a$ is excellent. The first twenty-six Wannier functions were used for the calculations.

\section{Conclusions about S-matrix combination}

From the results of the double bend and the simple Mach-Zehnder interferometer, we conclude that the Wannier function S-matrix calculations are self-consistent, i.e., the S-matrix combination gives similarly accurate results as the calculation of a monolithic circuit. 


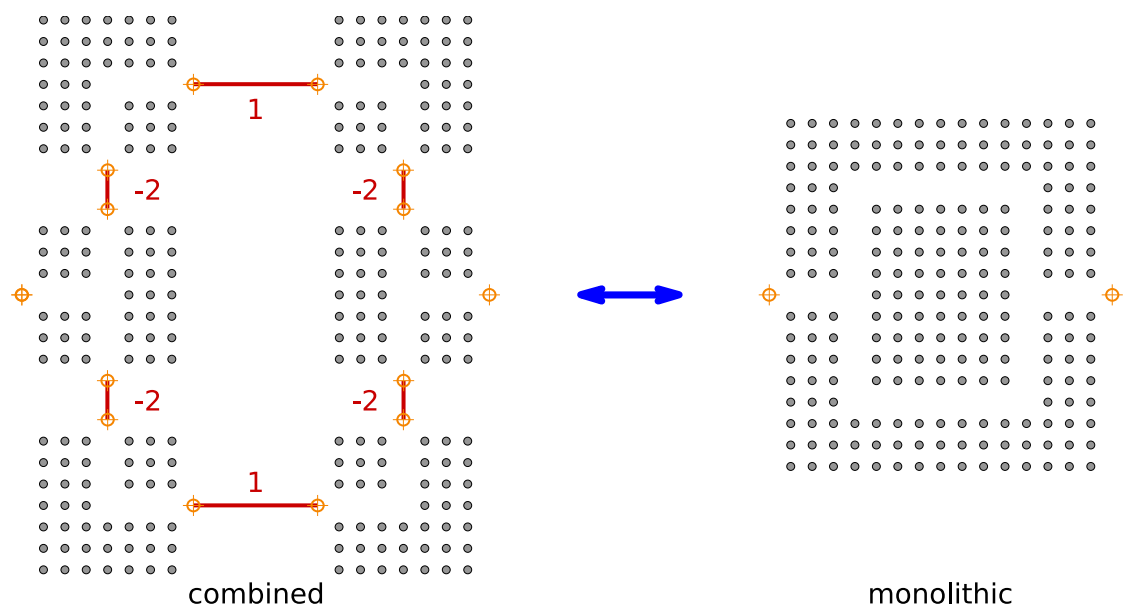

Figure 4.25.: Simple Mach-Zehnder interferometer in model system 1. On the left, it is decomposed into four bends and two T-splitters, each of which is represented by its individual S-matrix. By combining the S-matrices over waveguides of lengths $1 a$ and $-2 a$ (negative phase shift), respectively, the combined circuit S-matrix can be calculated. On the other hand, the monolithic circuit S-matrix can be calculated directly for one monolithic device.
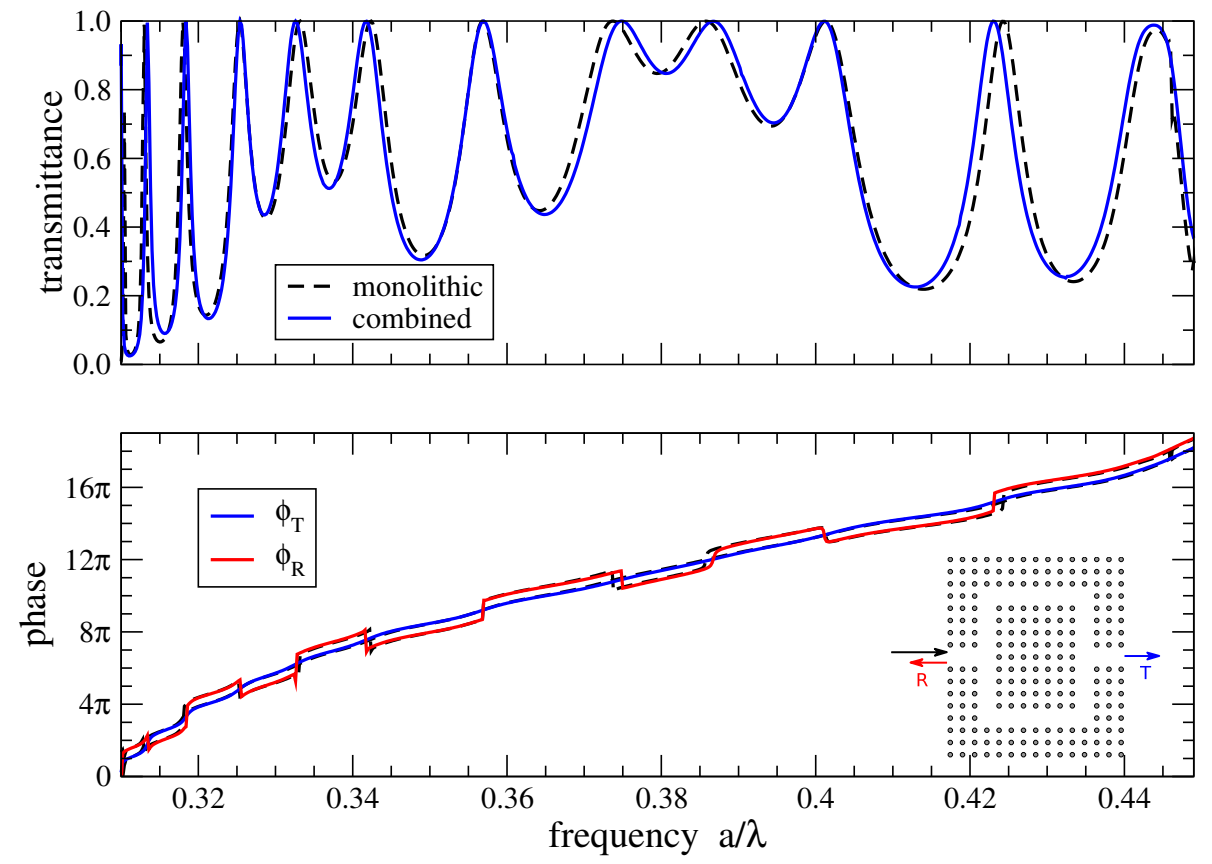

Figure 4.26.: Transmittance and phase spectrum of a simple Mach-Zehnder interferometer in model system 1 . Results calculated for the monolithic and the combined circuit are in very good agreement, both in transmittance and in phase. 


\section{Design of Large-Scale Circuits in a 2D Photonic Crystal}

In this chapter, we will discuss the design of large-scale circuits composed of basic functional elements (FEs) on the basis of model system 2 defined in section 4.1. First, we introduce the basic components (waveguides, optimized bends) for the circuits. Then we will gradually increase the circuit complexity and size, starting from one of the simplest compound devices, the directional coupler $(D C)$ presented in section 5.2. Within the scattering-matrix formalism, the length of the $D C$ is easily adjustable. This is a prerequisite for the design of more complex devices based on DCs, such as the Mach-Zehnder interferometer (MZI) discussed in section 5.3. Finally, we present an electrically tunable version of the MZI that can be used as a switch or modulator device.

\subsection{Basic Components}

First of all, for designing a particular circuit, we have to choose the underlying $\mathrm{PhC}$, which acts as a background isolating material for frequencies within its band gaps. Here, we take the $2 \mathrm{D}$ triangular lattice of air pores in silicon with radius $r=0.45 a$, introduced as model system 2 in section 4.1. This system is advantageous because of its large band gaps, especially for H-polarized light, and the overlap of band gaps for $\mathrm{H}$-polarization and E-polarization. In the following, we only make use of the large first band gap in H-polarization $\left(a / \lambda=[0.298,0.490], \Delta \omega / \omega_{c}=48.7 \%\right)$ and ignore the band structure for E-polarization in our considerations. For all calculations, we use the first twenty-six Wannier functions of model system 2 (cf. appendix B) with $R_{\max }=4$ according to $(2.26)$.

\subsubsection{Default Waveguide}

Besides the isolation provided by the band gap of the underlying $\mathrm{PhC}$, a $\mathrm{PhC}$ circuit needs "conductive paths" or, in terms of photonics, it needs waveguides. Typically, the bandwidth in which the circuit can be operated is mainly restricted by the bandwidth of the waveguides used in it. It is further reduced by requiring the waveguides to be single-mode. So it is important to make a good choice about the default waveguides used in the circuit.

In our model system using single-pore infiltration, the canonical choice for the default waveguide is a single line defect of pores filled with a low-index material such as a polymer with refractive index of $n=1.70$, for instance. The waveguide 


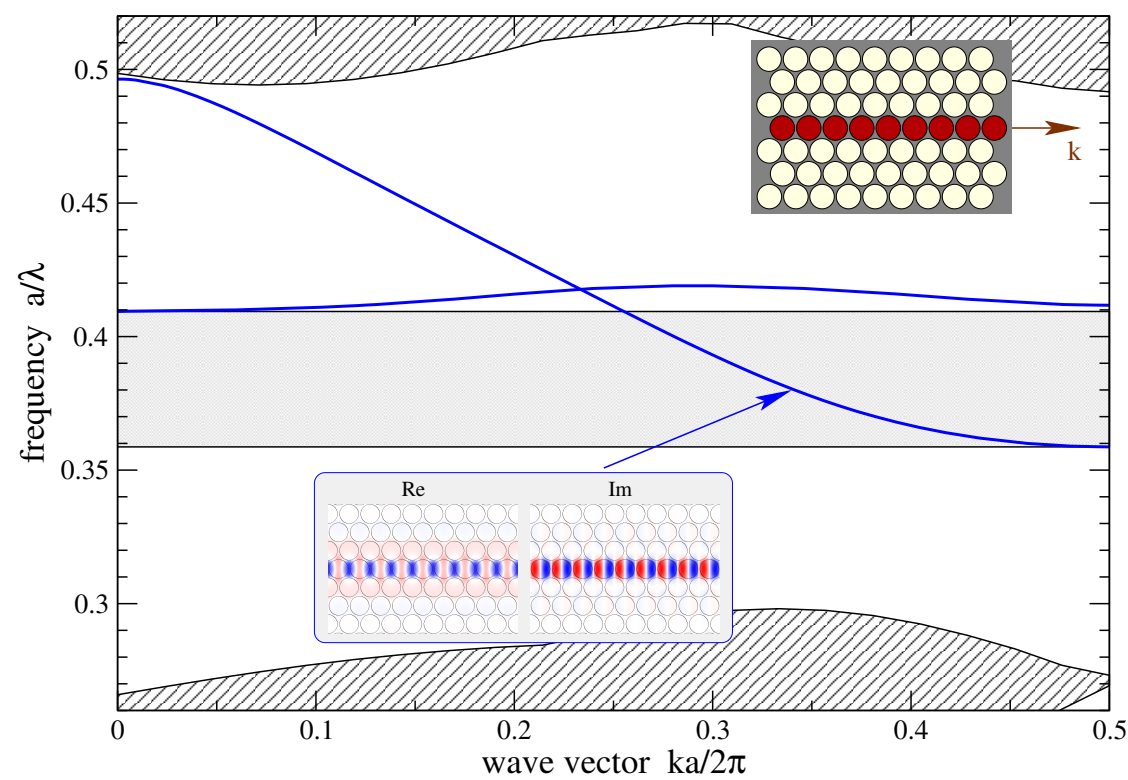

Figure 5.1.: Waveguide dispersion of the default waveguide for the circuit. The shaded region depicts the single-mode frequency range $(a / \lambda=[0.359,0.409])$, which is chosen for the operation of the circuit. The supported guided mode is symmetric with respect to the waveguide axis (see inset showing the periodic part of the mode).

dispersion of such a line defect is depicted in figure 5.1. The single-mode frequency range is identified as $a / \lambda=[0.359,0.409]$. In this range we choose to operate the circuit. It corresponds to a bandwidth of $\Delta \omega / \omega_{c}=12.7 \%$ with respect to the midgap frequency $\omega_{c}$, or $\Delta \lambda=211 \mathrm{~nm}$ for a midgap wavelength $\lambda_{c}=a / \omega_{c}=1550 \mathrm{~nm}$.

Fig. 5.1 also shows the periodic part of the guided mode for one frequency in the relevant frequency range. The mode is symmetric with respect to a mirror reflection at the waveguide axis. It is well confined to a narrow region around the waveguide.

\subsubsection{Optimized Bend}

The most important functional element for a $\mathrm{PhC}$ circuit is the waveguide bend. It occurs in almost every circuit and has to be designed such that internal reflections are avoided as much as possible. To fulfill this requirement, we optimize the waveguide bend with respect to minimal total reflectance over the frequency range of interest, allowing certain holes to be unfilled or filled with a material with variable refractive index. To be more specific, we search in the following configuration space, which is also depicted in figure 5.2:

- A range of $3 \times 3$ holes in the vicinity of the bend center may be unfilled or filled.

- The holes may be filled with different low-index materials with refractive indices of $n=1.55,1.60,1.65$ or 1.70 . 


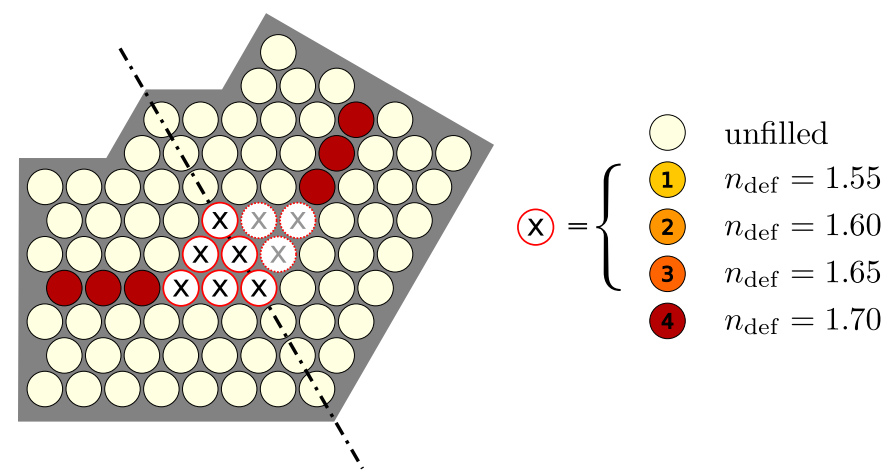

Figure 5.2.: Configuration space for the optimization of a waveguide bend for the $\mathrm{PhC}$ circuit. The holes depicted with " $x$ " may be unfilled or filled with low-index materials. Allowed configurations must be symmetric with respect to the axis depicted in the picture.

- Only symmetric configurations with respect to a mirror reflection on the plane bisecting the angle enclosed by the bend are taken into account.

This results in 5 different choices for the refractive index of each hole and a total of 6 holes that can be independently switched. Thus, we have a total number of $5^{6}=$ 15625 configurations to be scanned. The frequency range over which the reflectance is summed up is given by 5 discrete frequencies $a / \lambda=0.370,0.375,0.380,0.385$ and 0.390 .

Although we could use advanced techniques, such as simulated annealing, to solve this optimization task, the configuration space is not too large in the present case, so we used a simple brute-force optimization and computed all possible configurations. Since the Wannier function method allows to calculate a single configuration for a single frequency very efficiently, the task of computing all configurations for all frequencies was possible within a few days on a 32-processor cluster of AMD Opteron 248 processors. The first 26 Wannier functions were used on a specified device range (cf. figure 5.2). This resulted in a calculation time of approximately 1.5 minutes per configuration and frequency and a total computation time of approximately 62 hours on the 32-processor cluster for all configurations and all frequencies together. It has to be mentioned that the small-rank update procedure proposed in [99] (cf. section 2.3.4) could not be used here, since it is not efficient for such a high number of Wannier functions. This is because the singular-value decomposition and/or inversion of matrices involved in each small-rank update scales worse than the solution of the full system of equations for one configuration and, therefore, becomes slower at some point when increasing the number of Wannier functions.

The results of the optimization are shown in figure 5.3. The optimized bend has a reflectance below $10^{-2}=-20 \mathrm{~dB}$ in the frequency range $a / \lambda=[0.371,0.386]$. It has minimal reflectance at $a / \lambda=0.373$ and $a / \lambda=0.384$. The deep dips at these two frequencies will be useful for the following design of the directional couplers 


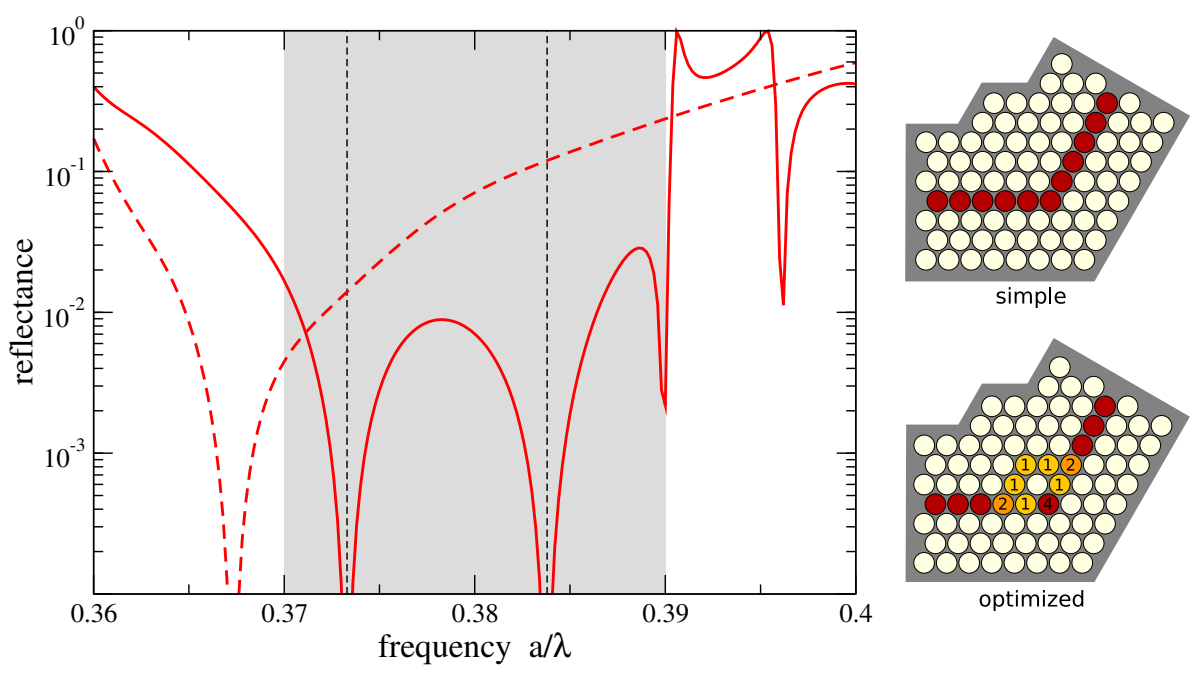

Figure 5.3.: Comparison of the performance of the simple and the optimized waveguide bend. The numbers in the sketch of the optimized bend correspond to different refractive indices according to figure 5.2. The vertical dashed lines depict the two frequencies with minimal reflectance of the optimized bend $(a / \lambda=0.373$ and $a / \lambda=0.384)$. They are repeated in subsequent spectra of the directional coupler and Mach-Zehnder interferometer.

and Mach-Zehnder interferometers, since those phase-sensitive devices work best if internal reflections are as small as possible. We will see that in frequency ranges where internal reflections can not be neglected, the large-scale circuit's performance deteriorates.

\subsection{Directional Coupler}

We start the discussion of large-scale circuit design in $\mathrm{PhCs}$ with a directional coupler (DC). The basic working principle of the DC was already introduced in section 1.3.1 for a conventional fiber DC. On the basis of the design choices of the underlying crystal and default waveguide, which we discussed in the previous section, we can sketch one possible realization of a $\mathrm{PhC} \mathrm{DC}$ in figure 5.4. Basically two choices have been made:

- The two waveguide lines are separated by two rows of unfilled holes.

- The optimized bend is utilized within the lower waveguide line.

In principle, many other DC designs could be investigated as well. Nevertheless, we restrict ourselves to the realization shown in figure 5.4 and focus on the length of the $\mathrm{DC}$ as the only remaining design freedom. 


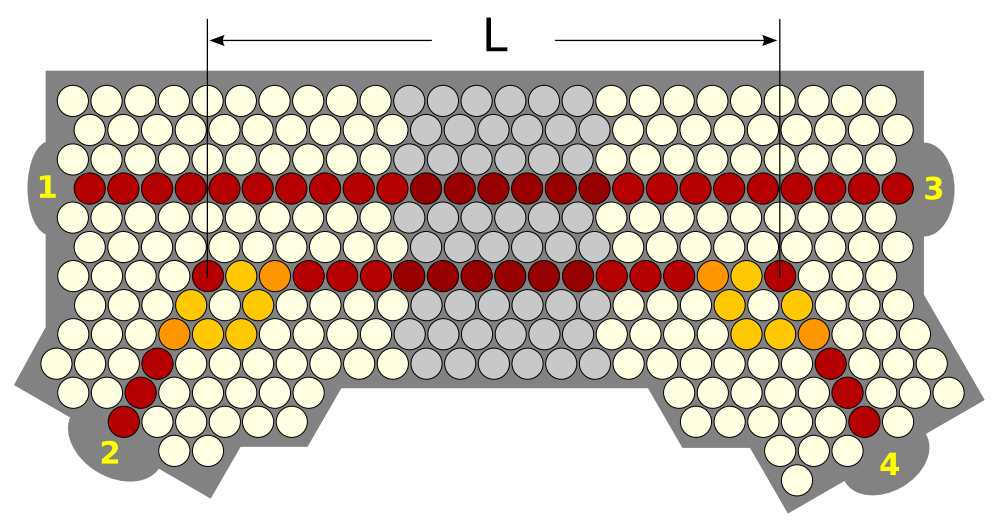

Figure 5.4.: Directional coupler built into a $\mathrm{PhC}$. It is decomposed into two coupler end pieces with a variable-length waveguide between them (gray-shaded region). We define the length $L$ of the coupler as the distance between the two bends in the lower arm.

\subsubsection{Additional Components}

We need two additional components for the description of the coupler depicted in Fig. 5.4 within the scattering matrix framework. The coupler consists of the two end pieces, which we will treat as functional elements, and of a coupling section between the end pieces, which is treated as a waveguide of variable length.

\section{Coupling-Section Waveguide}

Firstly, we have to calculate the dispersion and guided modes of the waveguide formed by the two parallel single-mode waveguides along the coupling section. This dualmode waveguide is less symmetric than a typical two-fiber coupling section (cf. section 1.3.1) due to the broken continuous translational symmetry of the underlying $\mathrm{PhC}$. However, it is still invariant under a glide reflection, i.e., a reflection on the axis equidistant from both single-mode waveguides in combination with a translation of $a / 2$ in the waveguide direction. Therefore, the two fundamental modes supported by the coupling-section waveguide are even and odd with respect to this glide reflection. Since they propagate with slightly different wave vectors $k_{\text {even }}$ and $k_{\text {odd }}$, a beat occurs when a superposition of both modes is excited. The waveguide dispersion and the mode profiles of the coupling-section waveguide are depicted in Fig. 5.5.

More precisely, the guided modes $\psi(\vec{r})$ of the coupling-section waveguide are even and odd with respect to the glide reflection only up to a phase $\phi$, which can be derived from the Bloch theorem,

$$
\hat{T}_{a} \psi(\vec{r})=\mathrm{e}^{\mathrm{i} k a} \psi(\vec{r}) .
$$

The glide reflection can be expressed by the successive application of a translation operator $\hat{T}_{a / 2}$ and a mirror operator $\hat{\sigma}$. Both operators are unitary and they commute, 


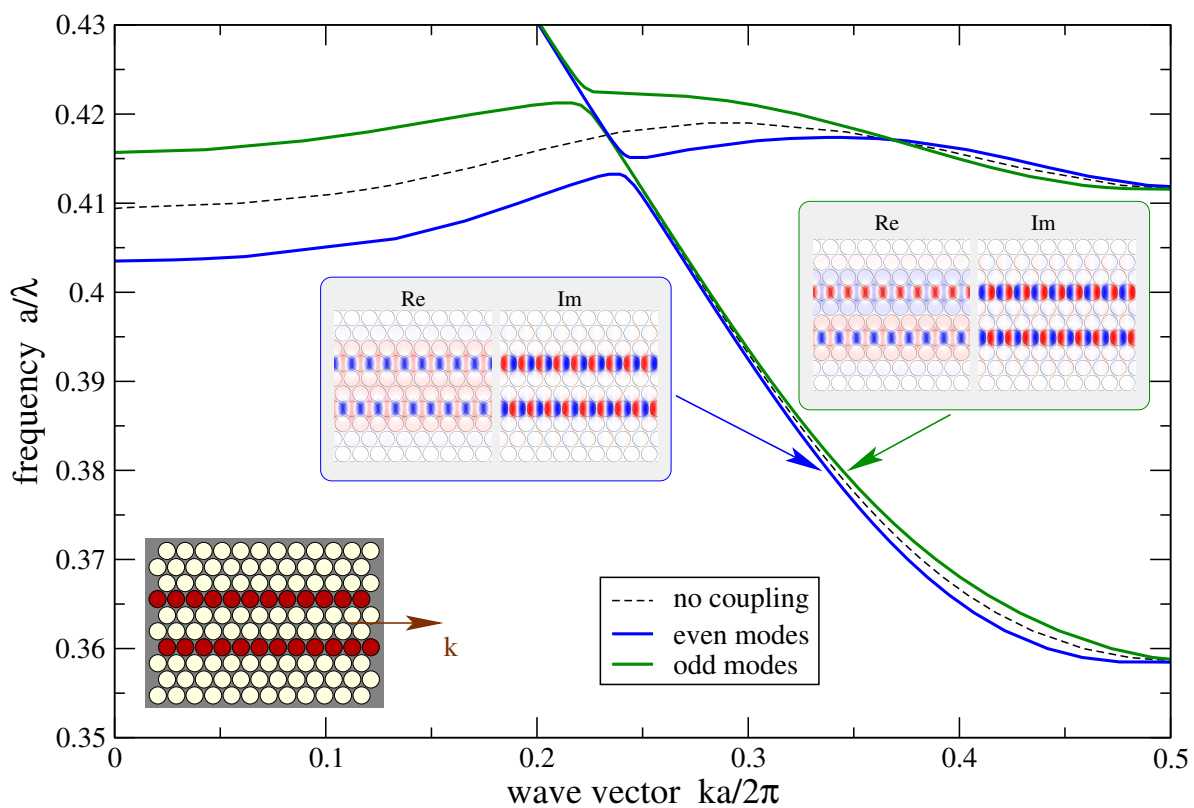

Figure 5.5.: Dispersion and mode profiles of the fundamental modes of the couplingsection waveguide, which is part of the directional coupler discussed here. For frequencies around $a / \lambda=0.38$, there exist two modes, an even and an odd mode with respect to a glide reflection, and they propagate with different wave vectors $k_{\text {even }}$ and $k_{\text {odd }}$. The dashed line represents the dispersion of the single-pore waveguide.

so the glide reflection operator is also unitary and has only eigenvalues of the form $\mathrm{e}^{\mathrm{i} \phi}$ (one-dimensional unitary representations),

$$
\hat{T}_{a / 2} \hat{\sigma} \psi(\vec{r})=\mathrm{e}^{\mathrm{i} \phi} \psi(\vec{r}) .
$$

On the other hand, the two-fold application of the glide reflection is equivalent to a simple translation by a distance of $a$, so that

$$
\begin{array}{cc}
\left(\hat{T}_{a / 2} \hat{\sigma}\right)^{2} \equiv \hat{T}_{a} \quad \Longrightarrow \quad \mathrm{e}^{2 \mathrm{i} \phi}=\mathrm{e}^{\mathrm{i} k a} \\
\Longrightarrow \quad \phi=\frac{k a}{2}+m \pi \quad m \in \mathbb{Z} .
\end{array}
$$

This phase relation of the guided modes of the coupling-section waveguide is important to correctly handle the phases of the S-matrix elements of different congruent coupler end pieces.

\section{Coupler End Piece}

The end pieces located at either side of the DC are treated as functional elements (FEs) that connect two single-mode waveguides with the coupling-section waveguide, 

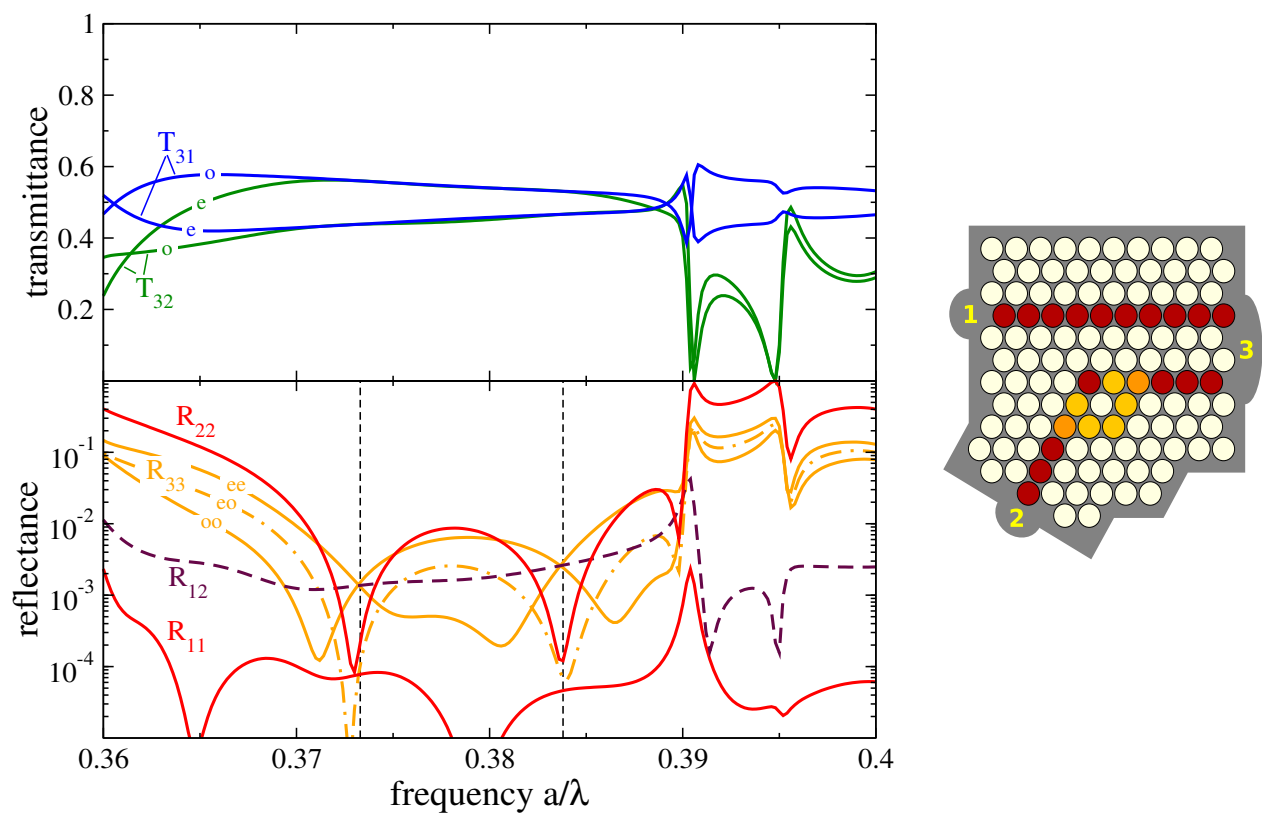

Figure 5.6.: Reflectance and transmittance spectrum of the coupler end piece depicted to the right. This is a three-port FE with a $4 \times 4 \mathrm{~S}$-matrix. The curves for matrix entries involving port 3 are labeled by letters 'e' for even and 'o' for odd, corresponding to the respective mode in port 3 . The optimized bend is incorporated into one arm and ensures very low reflectances at all three ports in the optimal range $a / \lambda=[0.371,0.386]$, including the "cross reflectance" $R_{21}$.

which supports two propagating guided modes. One representation of the coupler end piece and its reflectance and transmittance spectrum is shown in figure 5.6. It incorporates the optimized bend from the previous section in order to keep the reflectances as low as possible.

The coupler end piece is a three-port FE and is characterized in this case by a $4 \times 4$ S-matrix, according to the four propagating guided modes supported in the three ports. The S-matrix takes the form

$$
S_{\text {coupler }}=\left(\begin{array}{cccc}
r_{11} & & & \\
r_{21} & r_{22} & & \\
t_{31}^{(e)} & t_{32}^{(e)} & r_{33}^{(e e)} & \\
t_{31}^{(o)} & t_{32}^{(o)} & r_{33}^{(o e)} & r_{33}^{(o o)}
\end{array}\right),
$$

where the upper right part is left out, since it is equal to the lower left part due to reciprocity. We treat all S-matrix entries that connect the "left side" of the coupler end piece (ports 1 and 2) with the "right side" (port 3) and vice versa as transmission coefficients. All S-matrix entries that exclusively involve modes on either side are treated as reflection coefficients, including the "cross reflection coefficient" 
$r_{21}$. This somewhat unconventional labeling is natural for the present case, since an ideal coupler end piece has vanishing reflection coefficients, in the sense of the above definition, while the transmission coefficients include all features for the operation of the FE. In addition, we label the individual S-matrix entries related to port 3 with superscripts 'e' and 'o', depending on whether they are associated with the even mode or the odd mode in port 3 , respectively.

The transmittance spectrum in Fig. 5.6 basically shows two interesting features. Firstly, the transmittances into the even and odd modes in port 3 differ from each other. The coupling of an incoming mode in port 1 to the odd mode in port 3 is larger than its coupling to the even mode. The opposite is true for the coupling of an incoming mode in port 2, which is coupled more into the even mode. Secondly, except for exchanging the role of the even and the odd mode in port 3, the transmittances $T_{31}$ and $T_{32}$ are nearly equal in the optimal frequency range of the bend $(a / \lambda=$ $[0.371,0.386])$. Outside this range, $T_{32}$ deteriorates due to the worse transmittance through the bend.

Similarly, the reflectance spectrum in Fig. 5.6 shows features that can directly be attributed to the performance of the optimized bend. For example, the reflectances, including the "cross reflectance" $R_{12}$, stay well below $10^{-2}$ in the optimal frequency range of the bend. Furthermore, at the two frequencies $a / \lambda=0.373$ and 0.384 , where the reflectance of the bend goes to zero, the two diagonal elements in $R_{33}$ (even-to-even and odd-to-odd reflectance) and $R_{12}$ take all the same value and the non-diagonal terms in $R_{33}$ (odd-to-even reflectance and vice versa) go to zero. At these frequencies, ports 1 and 2 of the coupler end piece become balanced and there is no mechanism that couples even and odd modes in the coupling-section waveguide.

\subsubsection{Length-Dependent Performance and Spectrum}

Now that we have characterized the necessary waveguides and FEs involved in the directional coupler, we can analyze the entire DC as a circuit of two FEs. We first take the length $L$ of the coupling section, defined in Fig. 5.4, as a parameter and compute the length-dependent performance of the DC for one fixed frequency $a / \lambda=0.373$. At this frequency, the optimized bend (cf. section 5.1.2), which is incorporated into the coupler end piece, exhibits minimal reflectance. The results are depicted in Fig. 5.7. The DC nicely reproduces the behavior of a typical DC as described in the introduction (section 1.3.1). For instance, light entering port 1 is transmitted to port 3 ("bar transmittance" $T_{31}$ ) and port 4 ("cross transmittance" $T_{41}$ ), where the respective fractions depend on the length of the coupler. Therefore, the "bar transmittances" $T_{31}=T_{42}$ and the "cross transmittances" $T_{41}=T_{32}$ alternate when increasing the DC length. All reflectances (including the "cross reflectance" $R_{21}$ ) are below $10^{-2}$. There is a slight asymmetry between $T_{31}$ and $T_{41}$ that stems from the asymmetry created by the bends in the lower coupler arm. From Fig. 5.7, we can estimate the beat length to be $L_{B}=95 a$. This is in very good agreement with the expected value of $L_{B}=2 \pi /\left|k_{\text {even }}-k_{\text {odd }}\right|=94.1$ derived directly from dispersion data in Fig. 5.5. 


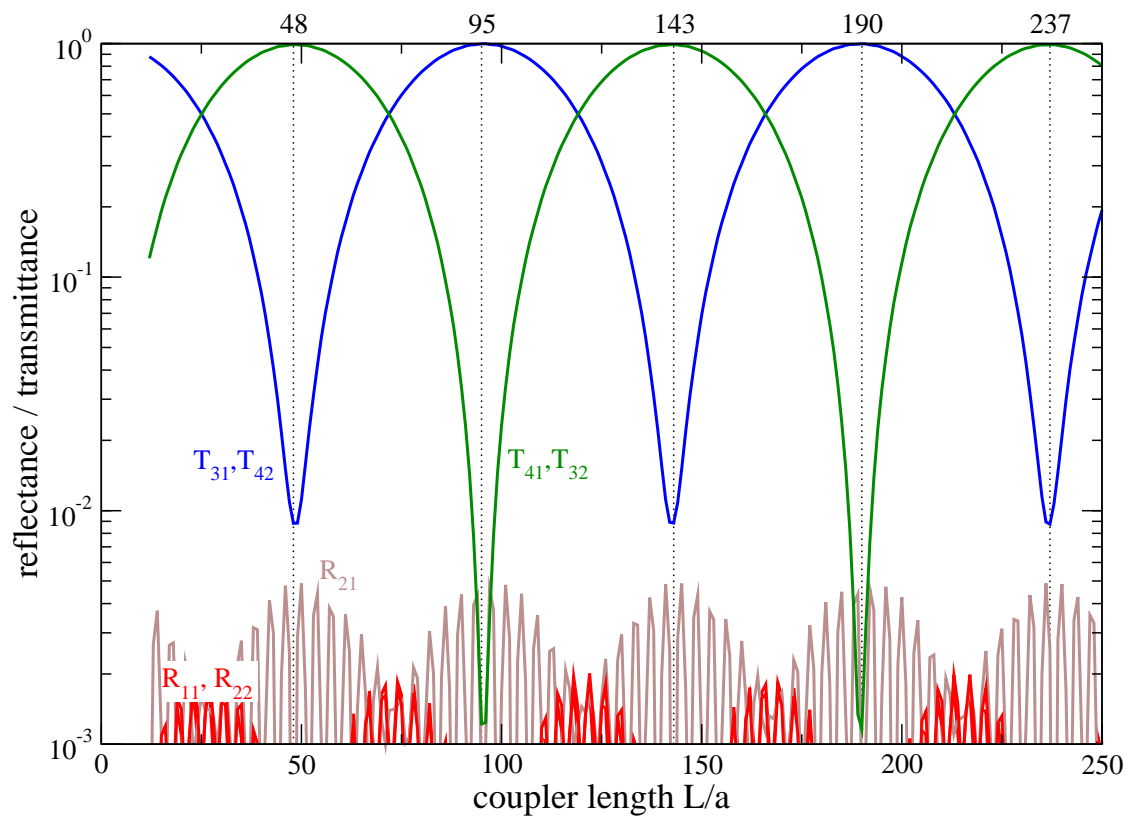

Figure 5.7.: Length-dependence of the reflectances and transmittances of the directional coupler shown in Fig. 5.4 for one fixed frequency $a / \lambda=0.373$. The "bar transmittances" $T_{31}=T_{42}$ and the "cross transmittances" $T_{41}=T_{32}$ alternate. The reflectances do not exceed $10^{-2}$.

The entire length-dependent performance of the DC can be computed easily, once the basic components are characterized. This means that we have to calculate the guided modes of the involved waveguides and the S-matrices of the coupler end pieces for one frequency. The congruence of the waveguides and coupler end pieces helps to reduce this effort. Then, the coupler length is tuned by only adjusting the length of the coupling-section waveguide when combining the S-matrices of the two opposite coupler end pieces. The actual effort to evaluate the S-matrix combination by means of Eq. (3.30) is negligible, it involves - for a given frequency - only $4 \times 4$ matrices. Therefore, the S-matrix approach is ideal for the characterization of circuits, in which the length of connections acts as a parameter.

Instead of varying the length of the DC for a fixed frequency, we now calculate the frequency spectrum of a fixed-length DC. The results are plotted in Fig. 5.8. Since the transmittance of an incoming field into a specific output port depends on the ratio of the coupler length and the beat length, and the beat length $L_{B}(\lambda)$ is wavelengthdependent, we get an oscillating behavior of the transmittances with frequency. This is at least the case in frequency regions where the reflectances ("back reflectances" $R_{11}$ and $R_{22}$ as well as "cross reflectance" $R_{21}$ ) are small. In other frequency regions, the device performance is mainly governed by Fabry-Perot oscillations due to nonnegligible reflectances and resonant behavior. These effects can completely destroy the characteristic features of a DC. Again, we identify the optimal frequency range 


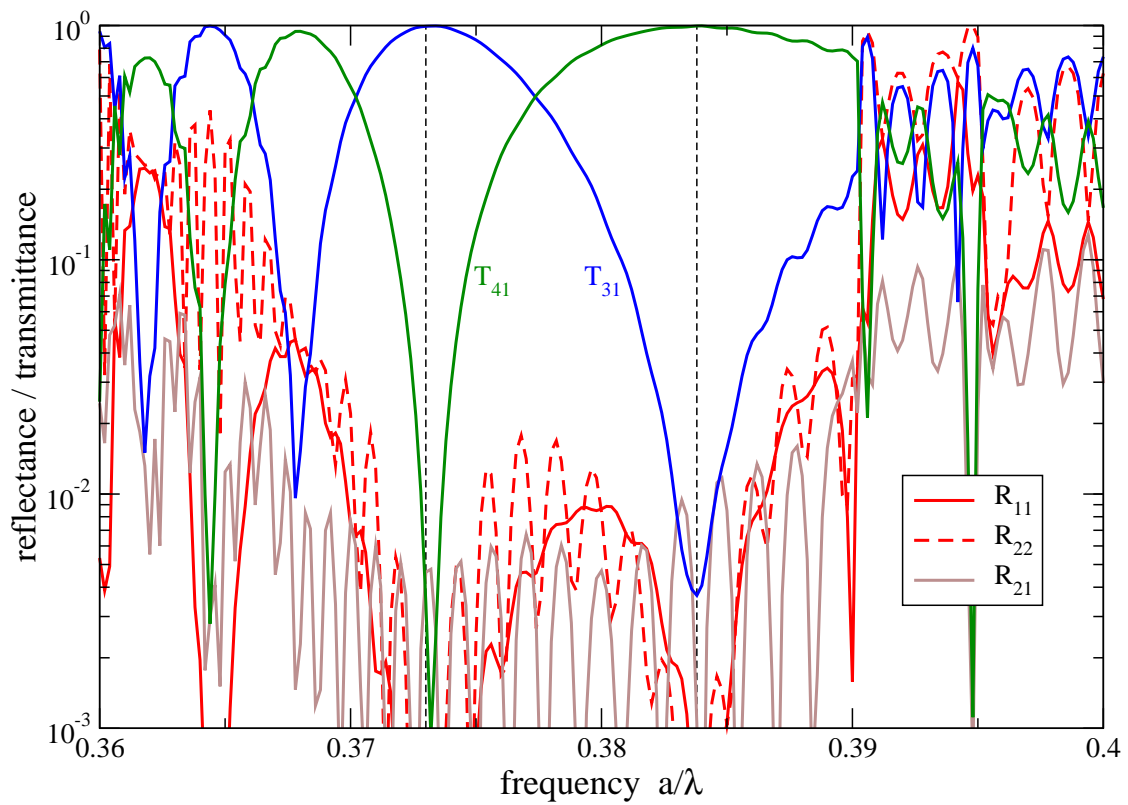

Figure 5.8.: Reflectance/transmittance spectrum of a fixed-length DC as shown in Fig. 5.4 with $L=97 a$. For this length, the DC is in the bar state (maximum transmittance along one arm $1 \rightarrow 3$ or $2 \rightarrow 4$ ) for $a / \lambda=0.373$ and in the cross state (maximum transmittance across the two arms) for $a / \lambda=0.384$.

$a / \lambda=[0.371,0.386]$ of the bend (cf. section 5.1.2), in which the DC can be operated with good performance.

Note that for the frequency spectrum, the guided modes of the involved waveguides and the S-matrices of the coupler end pieces have been calculated for each frequency. No additional data would be necessary to calculate the length-dependent performance of the DC for each frequency.

\subsection{Mach-Zehnder Interferometer}

On the basis of the DC discussed in the previous section, our next goal is to design and characterize a Mach-Zehnder interferometer in a 2D PhC. We specify that the MZI consists of two DCs as designed in the previous section, which are interconnected via two separate arms. One arm (or both) may include a delay line to create relative phase shifts between the two arms. A sketch of the device is shown in Fig. 5.9.

\subsubsection{Additional Components}

Besides the optimized bend and the DC components, we need two additional elements for the description of delay lines that may appear in the arms of the MZI. Those components are (i) the waveguide that forms the delay line (called delay-line 


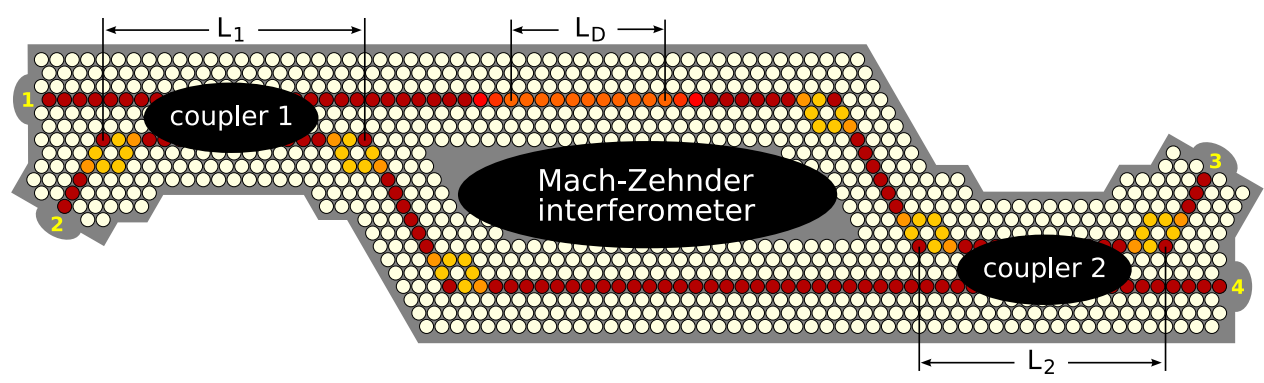

Figure 5.9.: Sketch of a Mach-Zehnder interferometer (MZI) in a 2D PhC. It consists of two couplers whose ports are interconnected via two separate arms. A delay line is incorporated in the upper arm by filling the pores of the waveguide with a different material over a certain length.

waveguide) and (ii) an additional FE to account for the transitions from the standard waveguide to the delay-line waveguide and back. Due to the congruence of the two transitions, only one FE is necessary to describe both of them.

\section{Delay-Line Waveguide}

By definition, a delay-line waveguide must have a dispersion different from the dispersion of the standard waveguide. Thus, a delay line of a certain length $L_{\mathrm{D}}$ creates a relative phase shift $\Delta \phi$ as compared to a standard waveguide of the same length. Since we want to stay in the framework of single-pore infiltration, the most straightforward choice for composing the delay-line waveguide is by using a different infiltration material as compared to the standard waveguide fill material. Therefore, the only design freedom we retain is the contrast of the refractive indices of the delay-line and standard waveguide fill materials $n_{\mathrm{D}}: n_{\text {ref }}$.

There are two constraints that constrict good choices for the index contrast. The stronger it is, the shorter the delay line needs to be to achieve a certain phase shift. To minimize the device size, a strong index contrast is favorable. On the other hand, within the $\mathrm{PhC}$, the delay-line length $L_{\mathrm{D}}$ can only be increased or decreased by integer multiples of the lattice constant. Therefore, $\Delta \phi$ is also quantized. The steps in which $\Delta \phi$ can be increased or decreased are smaller for weaker index contrast. Therefore, a weak index contrast is favorable for fine-tuning of the delay line's relative phase shift.

A good compromise between these two constraints is an index contrast of $n_{\mathrm{D}}$ : $n_{\text {ref }}=1.65: 1.70$. Fig. 5.10 shows the dispersion of such a delay-line waveguide in comparison to the standard waveguide. The step in which $\Delta \phi$ is increased per unit length of the delay line lies in the range of $\Delta k a \approx[0.08 \pi, 0.04 \pi]$ in the frequency range $a / \lambda=[0.37,0.39]$. This corresponds to delay-line lengths of $L_{\mathrm{D}} \approx 12 a$ to $25 a$ to achieve a relative phase shift of $\Delta \phi=\pi$ at the respective frequencies. 


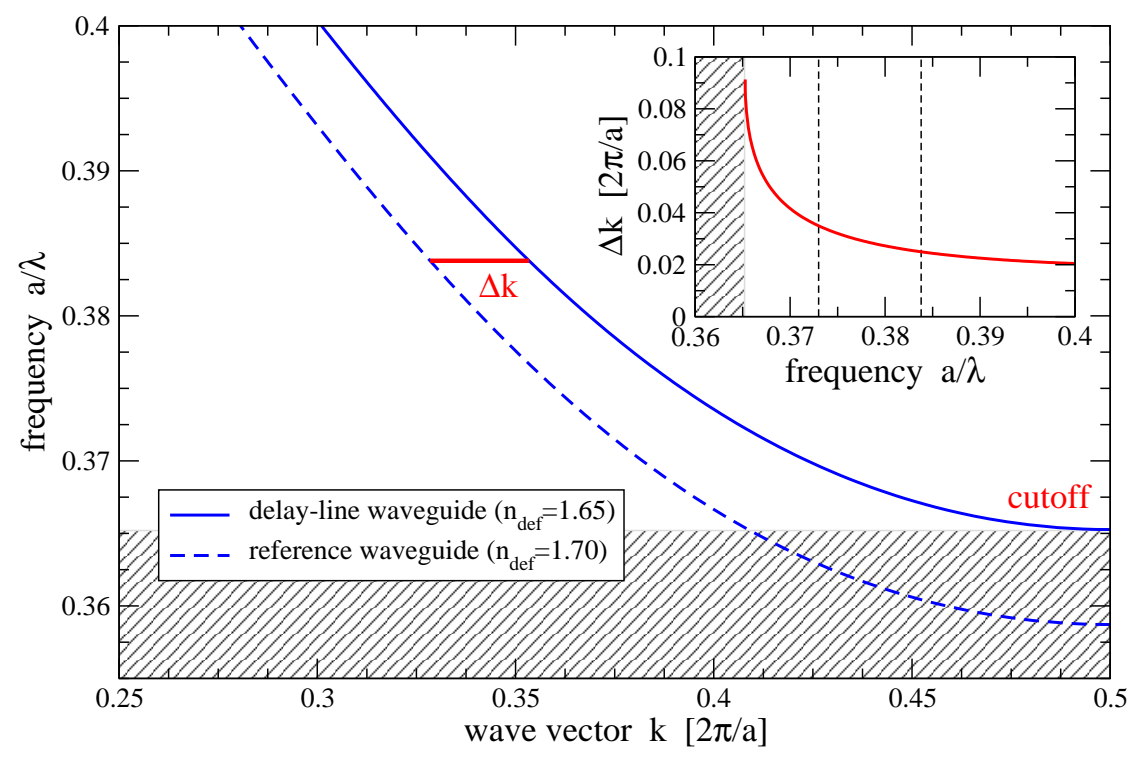

Figure 5.10.: Comparison of the dispersion of a delay-line waveguide $\left(n_{\text {def }}=1.65\right)$ and the default waveguide $\left(n_{\text {def }}=1.70\right)$. The difference in wave vector $\Delta k$ at a given frequency determines the relative phase shift $\Delta \phi$ per unit length of the delay line.

\section{Delay-Line Transition}

Besides the delay-line waveguide, the delay-line is formed by a transition from the standard to the delay-line waveguide and a transition back, which are handled as simple two-port FEs. Both FEs are described by the same S-matrix, since they are congruent. Therefore, we only need to compute the S-matrix of one transition.

An ideal delay line would work like a reflectionless phase shifter, so we design the delay-line transitions such that they are smooth enough to get low reflectance over a broad frequency range. Fig. 5.11 shows the reflectance of two instances, an abrupt and a smooth transition. In the latter, two holes are filled with materials of intermediate refractive index, $n_{\text {def }}=1.667$ and 1.683. Although the reflectance of the abrupt transition is not that bad, the smooth transition has even more reduced reflectance. In the following, we choose the smooth transition for the MZI calculations.

\subsubsection{Balanced and Unbalanced $\mathrm{MZI}$}

Next, we put all components together and characterize the full MZI depicted in Fig. 5.9. In this large-scale circuit, we have three adjustable design parameters: the lengths $L_{1}$ and $L_{2}$ of the two couplers involved in the circuit and the length $L_{\mathrm{D}}$ of the delay line in one of the MZI arms. To get an idea of the MZI performance with respect to the different lengths, we first consider an MZI in which the delay line is left out completely, and we scan through the two coupler lengths $L_{1}$ and $L_{2}$. This case represents a balanced MZI, in which the two arms have equal optical path lengths. 


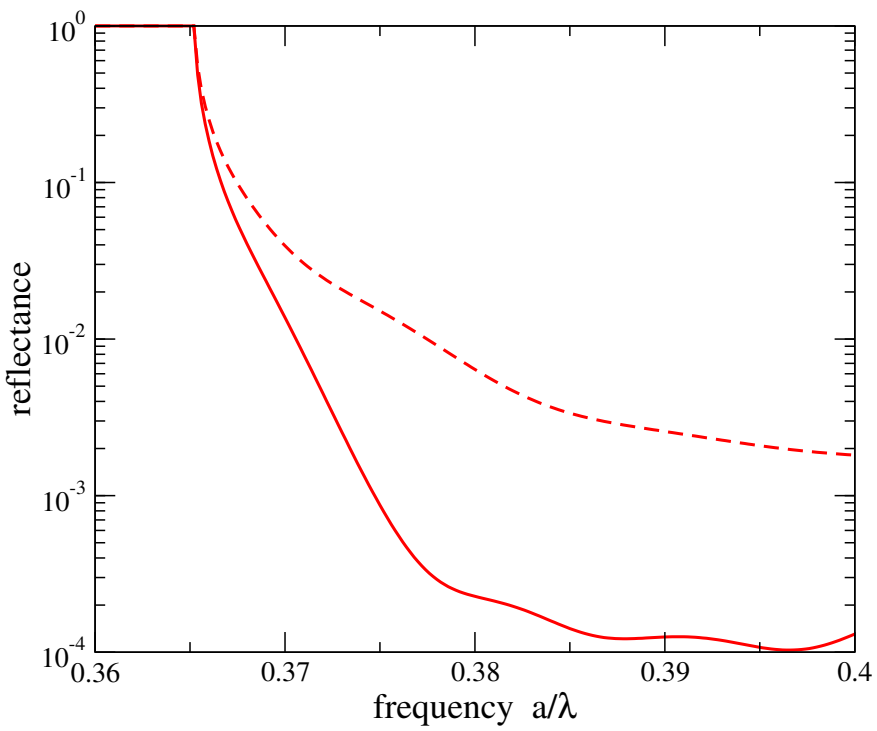

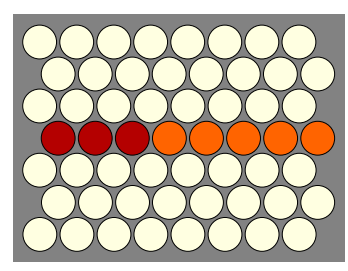

abrupt

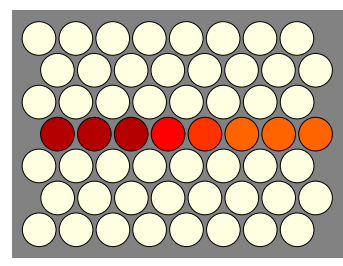

smooth

Figure 5.11.: Reflectance of the abrupt and the smooth delay-line transition between the default waveguide $\left(n_{\text {def }}=1.70\right)$ and the delay-line waveguide $\left(n_{\text {def }}=1.65\right)$. In the smooth transition, two holes are filled with materials of intermediate refractive index $\left(n_{\text {def }}=1.667\right.$ and 1.683).

Fig. 5.12 gives an overview about the results of the parameter scan for $L_{1}$ and $L_{2}$. The balanced MZI is equivalent to a DC of length $L_{1}+L_{2}$. This is because light does not accumulate relative phases along the two arms, so that the exit phase relation at coupler 1 is the same as the input phase relation at coupler 2 . Thus, the effects of both couplers simply add up. The transmittance spectrum of a specific realization of the balanced MZI $\left(L_{1}=L_{2}=49 a\right)$ is plotted in Fig. 5.13. We have chosen this particular combination of lengths because a DC of length $L=97 a$ is in the cross state $\left(T_{41} \approx 1.0\right)$ for the frequency $a / \lambda=0.384$ (cf. Fig. 5.8). Consequently, the balanced MZI with $L_{1}+L_{2}=98 a$ shows a very similar overall behavior. This can be verified by directly comparing Figs. 5.13 and 5.8.

In the next step, we incorporate the delay line into one arm and thereby create an unbalanced MZI, in which the optical path length of one arm is larger than that of the other arm. This induces a relative phase shift $\Delta \phi$ for light travelling along the modified arm. The phase shift for a fixed delay-line length is frequency-dependent. In Fig. 5.14 we plot the spectrum of $\Delta \phi$ for three different delay-line lengths, $L_{\mathrm{D}}=$ $6 a, 10 a$, and $17 a$. We see that $\Delta \phi$ varies slowly within the shown frequency range. Note that for $L_{\mathrm{D}}=17 a$, the relative phase shift is $\Delta \phi=\pi$ at the frequency $a / \lambda=$ 0.384. In an unbalanced MZI with two identical couplers of equal lengths $L_{1}=L_{2}$, a relative phase shift of $\pi$ is special, since for this case, the effect of the first coupler on the incoming field is exactly reverted by the second one, if the couplers are sufficiently close to ideal. This means that the light always exits in the arm it was launched. 


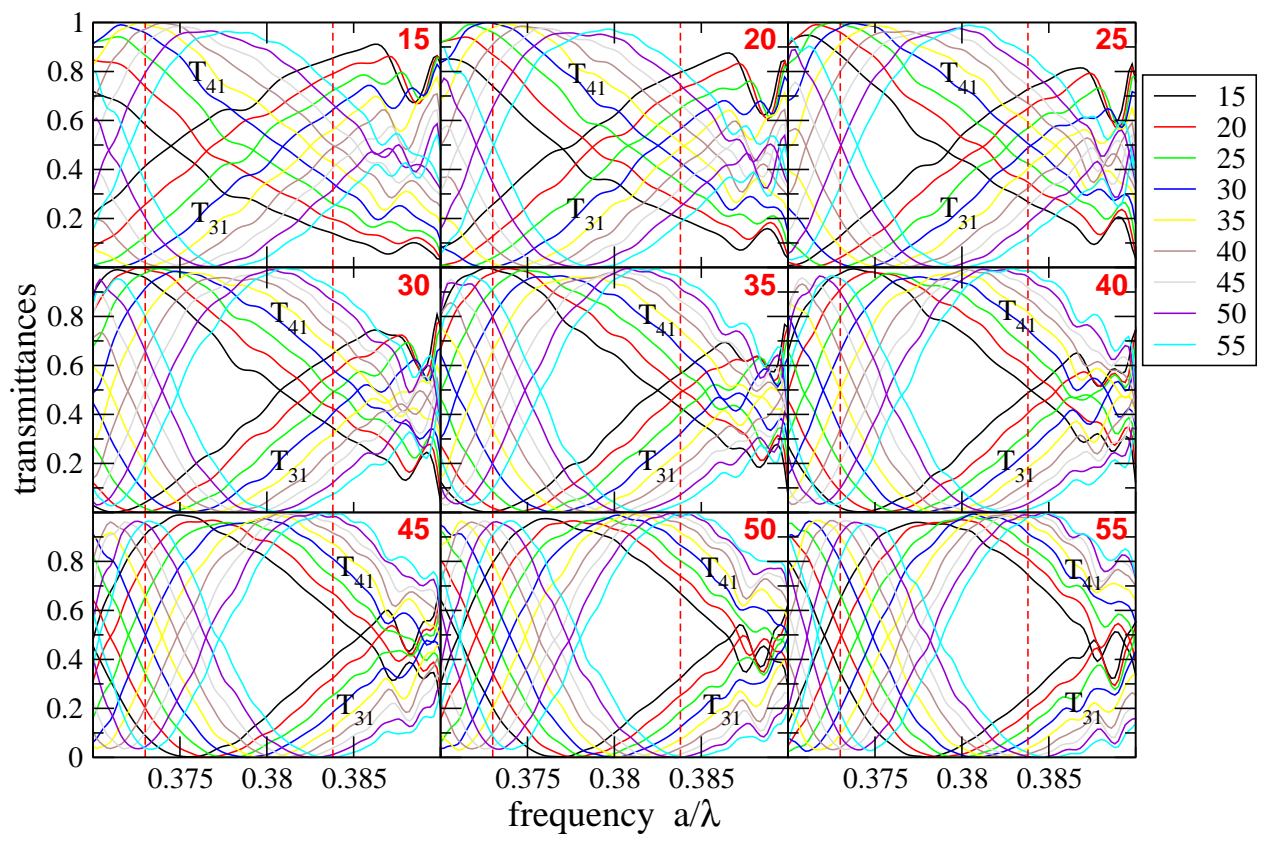

Figure 5.12.: Parameter scan for the balanced MZI. Transmittance spectra are plotted for different lengths $L_{1}$ and $L_{2}$ of the couplers. The red number in each subgraph corresponds to $L_{1}$, while $L_{2}$ is represented by the color of each curve.

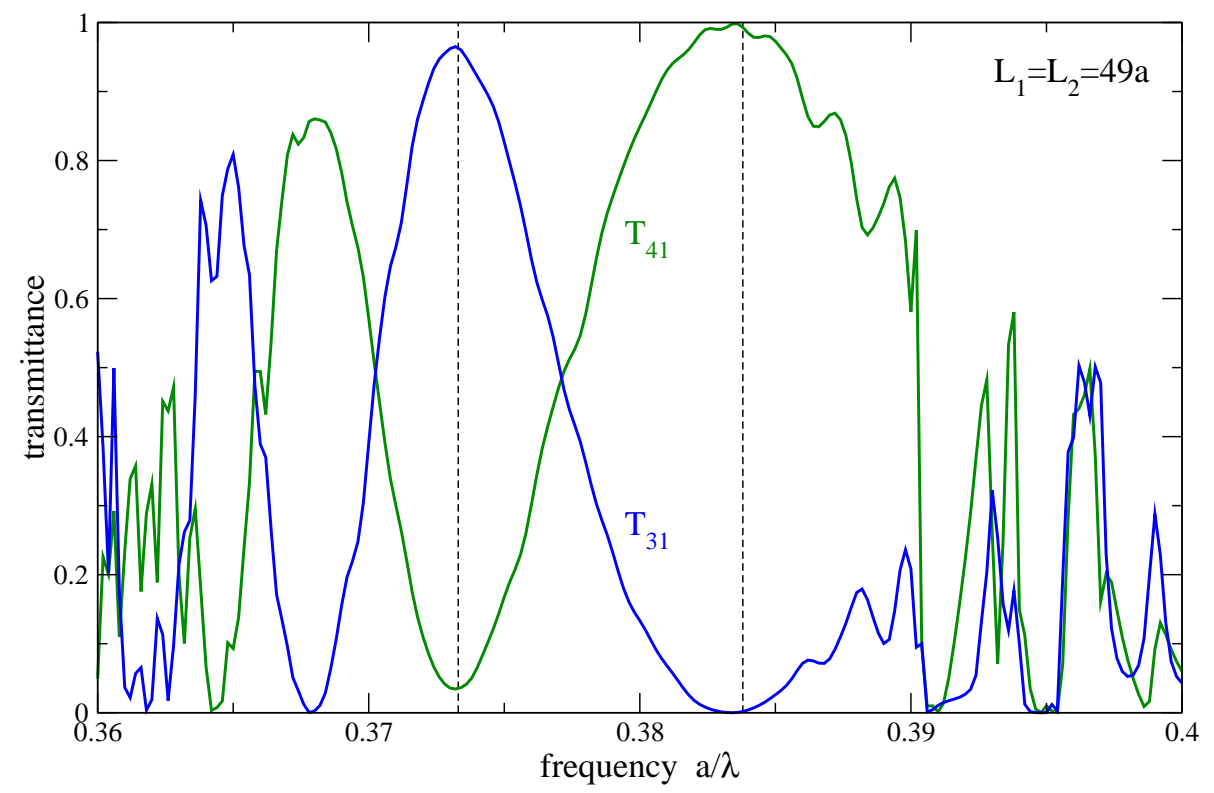

Figure 5.13.: Transmittance of the MZI depicted in figure 5.9 with $L_{1}=L_{2}=49 a$ without the delay line (both arms have the same optical length). This balanced MZI performs similar to a DC of length $L_{1}+L_{2}=98 a$. Compare with figure 5.8. 

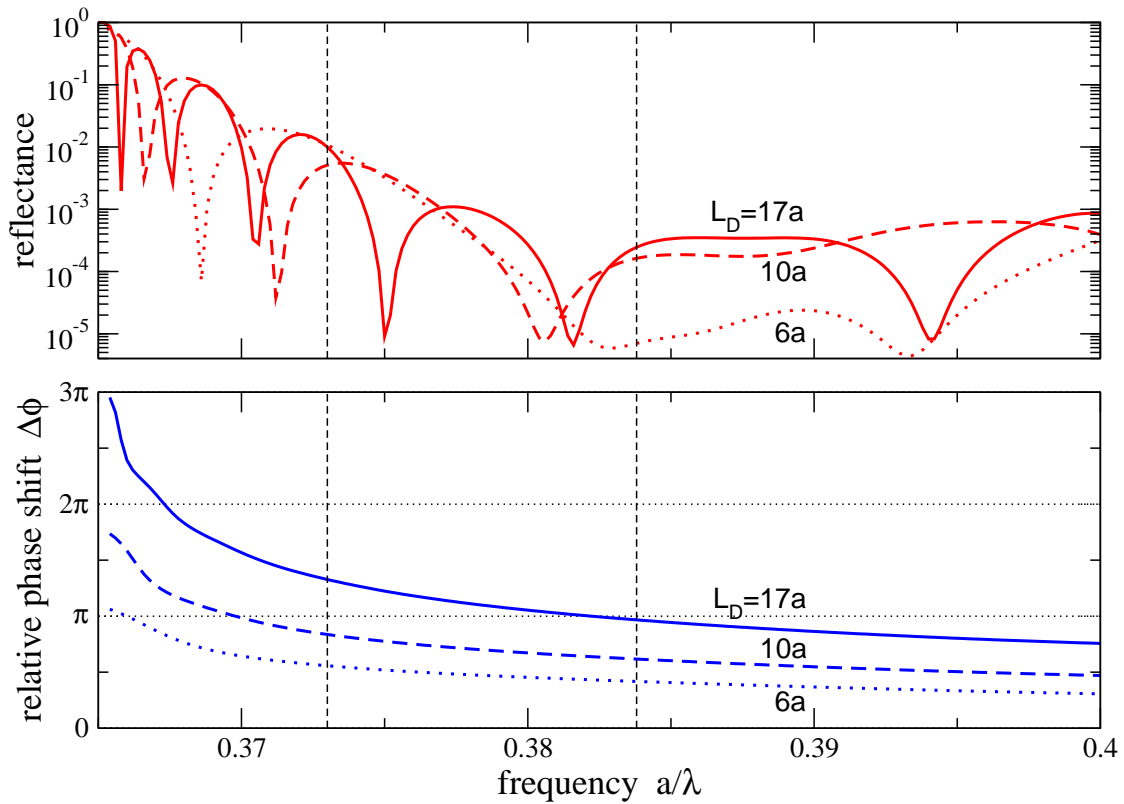

Figure 5.14.: Spectra of the reflectance and relative phase shift $\Delta \phi$ for delay lines (with smooth transitions) of different lengths $\left(L_{\mathrm{D}}=6 a, 10 a, 17 a\right)$.

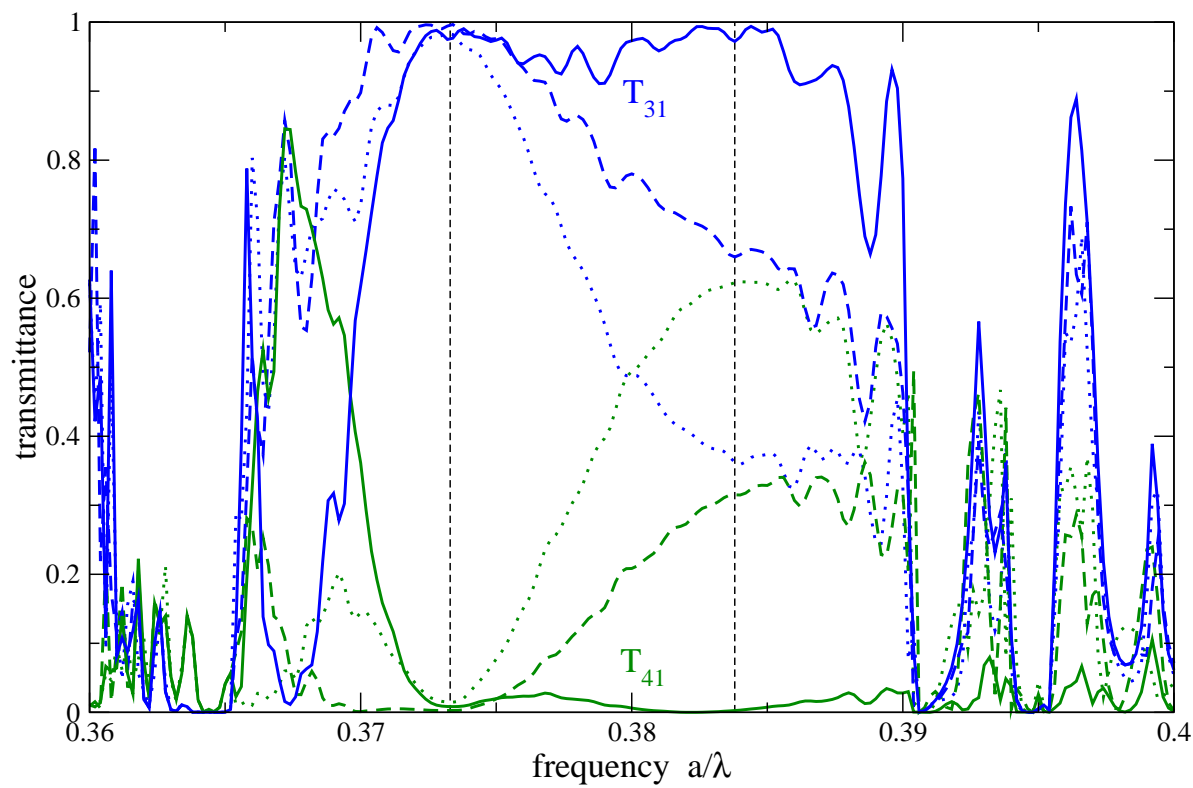

Figure 5.15.: Transmittance of the MZI depicted in figure 5.9 with $L_{1}=L_{2}=49 a$ and a delay line of variable length $L_{\mathrm{D}}=17 a$ (solid curves), $10 a$ (dashed curves) and $6 a$ (dotted curves). For $L_{\mathrm{D}}=17 a$, the relative phase shift approximately corresponds to $\Delta \phi=\pi$ at $a / \lambda=0.384$, leading to $T_{31} \approx 1.0$. For shorter delay lines, it takes some value $0<\Delta \phi<\pi$ at $a / \lambda=0.384$, so the transmittances tend to change towards the results for the balanced MZI. Compare with Fig. 5.13 ( $\Delta \phi=0$ for all frequencies). 


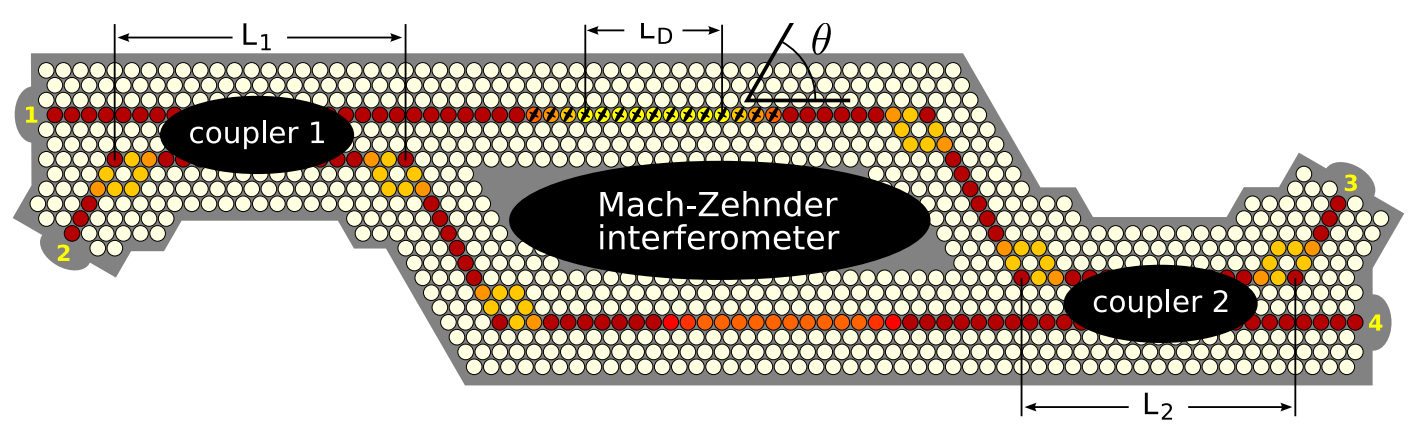

Figure 5.16.: Actively tunable MZI incorporating a liquid-crystal filled delay line in the upper arm. The lower arm includes a reference delay line whose length is always adjusted to the length $L_{\mathrm{D}}$ of the $\mathrm{LC}$ delay line. The LC director angle $\theta$ represents the tuning parameter for the MZI.

\subsection{Actively Tunable Mach-Zehnder Interferometer}

We now go one step further and design an MZI with tunable relative phase shift between the two arms. It is illustrated in Fig. 5.16. The tunability is provided by a liquid-crystal (LC) filled delay line in one arm, where the LCs can be orientated by applying an external electric field. In the other arm, we add a reference delay line that has (almost) the same dispersion as the LC delay line for a certain director angle $\theta=60^{\circ}$ of the LCs. So we can tune the relative phase shift to values $0 \leq \Delta \phi \leq$ $\Delta \phi^{(\max )}$ by adjusting the director angle. The maximum relative phase shift $\Delta \phi^{(\max )}$ we can achieve grows with the delay line length. The resulting circuit can be used as a electrically operated optical switch or modulator.

\subsubsection{Additional Components}

Besides the passive MZI components we discussed above, we need to describe the tunable delay line, which includes anisotropic LCs. As briefly mentioned in section 1.2.5, the Wannier function approach can handle anisotropic materials [76]. We treat the LC-filled pores like normal point defects. The director angle $\theta$ is specified as a parameter. The tunable delay line is split into two FEs, the transitions to and from the delay-line, and the tunable delay-line waveguide. Due to the congruence of the transitions, we only need to characterize one transition.

\section{Tunable Delay Line}

In our calculations, we use LCs with ordinary and extraordinary refractive index $n_{\mathrm{o}}=1.5$ and $n_{\mathrm{e}}=1.7$, respectively, for the tunable material incorporated in the delay line. Analogous to the static delay line components discussed in section 5.3.1, we smooth the transitions by filling three pores with LCs of intermediate refractive index $\left(n_{\mathrm{e}}=1.7, n_{\mathrm{o}}=1.55,1.60,1.65\right)$ to ensure low reflectance at the transitions. 


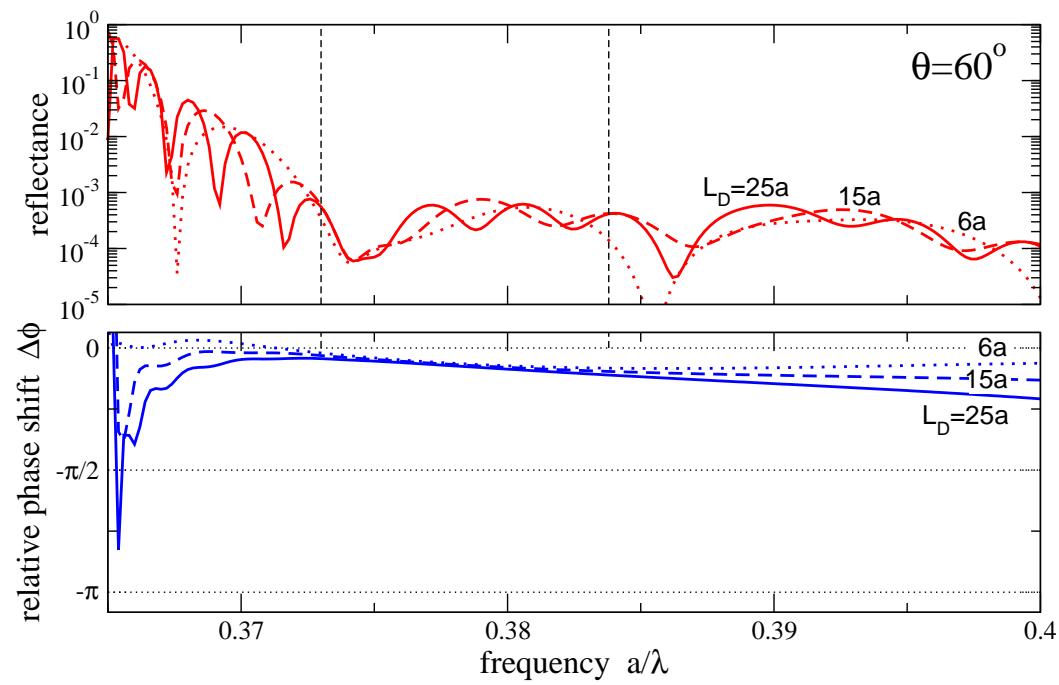

Figure 5.17.: Reflectance and relative phase shift for a tunable delay line with LC director angle $\theta=60^{\circ}$. The dispersion of the LC delay line is almost identical to the dispersion of the reference delay line. Therefore, the relative phase shift is very small and the length of the delay lines is not important.

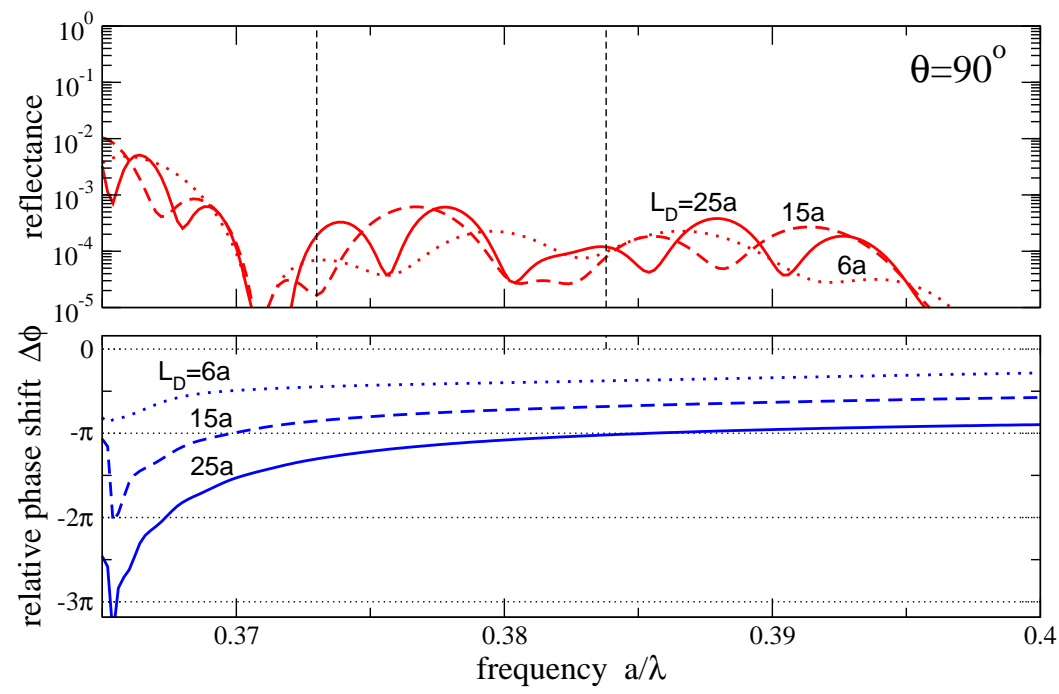

Figure 5.18.: Reflectance and relative phase shift $\Delta \phi$ for a tunable delay line with LC director angle $\theta=90^{\circ}$. The dispersions of the $\mathrm{LC}$ delay line and the reference delay line differ. Therefore, $\Delta \phi$ varies with frequency and can be increased or decreased by adjusting the length $L_{\mathrm{D}}$ of the delay lines.

The dispersion of the delay-line waveguide can be tuned by adjusting the director angle $\theta$ of the LCs. Figs. 5.17 and 5.18 show the reflectance and relative phase shift $\Delta \phi$ for LC delay lines of different lengths. $\Delta \phi$ is calculated with respect to the reference delay line, whose length is always increased or decrease by the same 


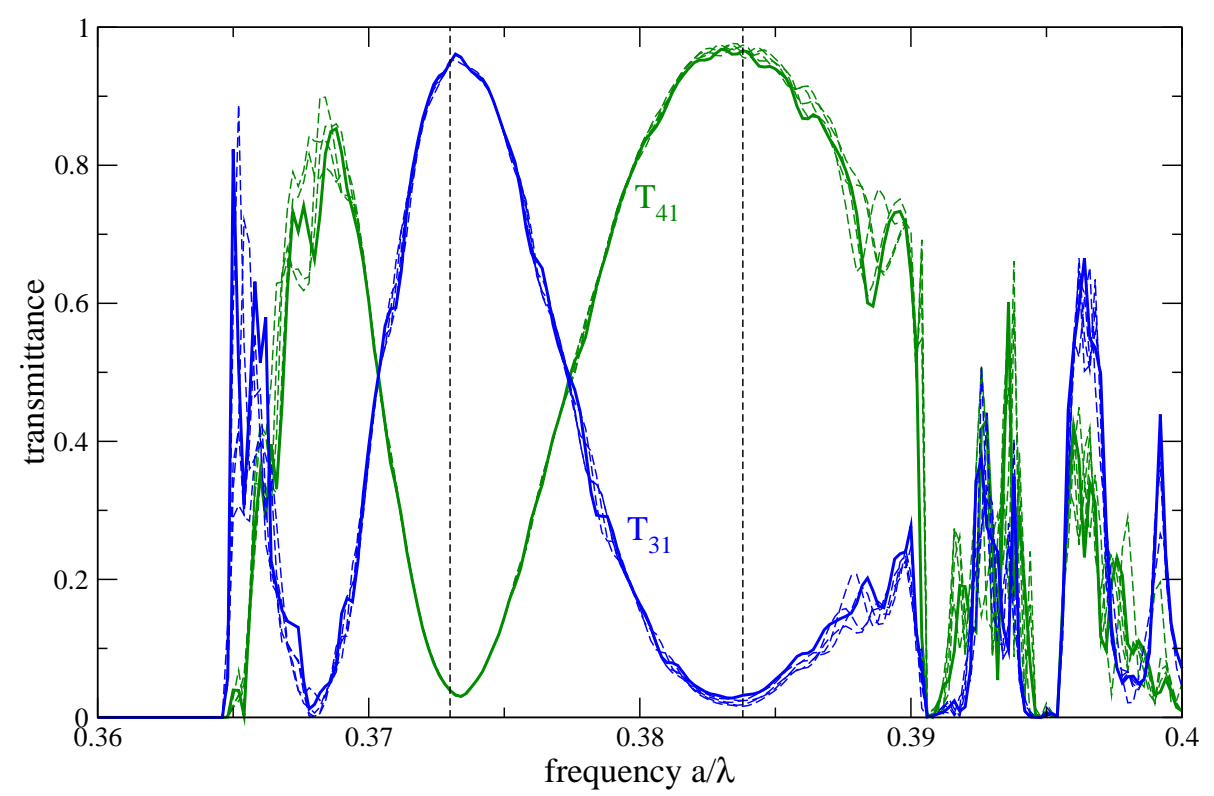

Figure 5.19.: Transmittance spectrum for the actively tunable MZI with a director angle $\theta=60^{\circ}$ in the liquid-crystal delay line with length $L_{\mathrm{D}}=25 a$ (solid curve). Since both delay lines have nearly the same dispersion, this case is comparable to the balanced MZI discussed in Fig. 5.13. The effect of changing the delay line length (dashed curves, $L_{\mathrm{D}}=6 a, 10 a, 15 a, 20 a$ ) is very small.

amount as $L_{\mathrm{D}}$ is. See Fig. 5.16 for the absolute lengths of the delay lines relative to each other, including transitions. The dispersion of the LC delay line for $\theta=60^{\circ}$ is almost equal to the dispersion of the reference delay line, so the relative phase shift for $\theta=60^{\circ}$ is nearly zero for all frequencies. On the other hand, the LC delay line generates a relative phase shift for $\theta=90^{\circ}$, which varies slowly with frequency.

\subsubsection{Application: Switch or Modulator}

Putting it all together, we characterize the tunable MZI depicted in Fig. 5.16 with respect to the design parameters $L_{1}, L_{2}$ and $L_{D}$. We take two values for the tuning parameter (the director angle), $\theta=60^{\circ}$ and $90^{\circ}$. The design goal is that the circuit behaves like a balanced MZI for $\theta=60^{\circ}$ and like an unbalanced MZI with $\Delta \phi=\pi$ at a given frequency, $a / \lambda=0.384$, for $\theta=90^{\circ}$. The lengths $L_{1}$ and $L_{2}$ of the two couplers involved in the MZI can be adopted from the designs in section 5.3, where we found that $L_{1}=L_{2}=49 a$ is suitable. For $\theta=60^{\circ}$, the delay line length $L_{D}$ is irrelevant, so the only design parameter remaining is the delay line length $L_{D}$ for $\theta=90^{\circ}$. In Fig. 5.18, we see that a delay line with length $L_{D}=25 a$ causes a relative phase shift of $\Delta \phi=\pi$ at $a / \lambda=0.384$. Therefore, this length fulfills our design requirements. In Figs. 5.19 and 5.20, the transmittance spectrum is plotted for $\theta=60^{\circ}$ and $90^{\circ}$, respectively. It is apparent that the bar transmittance $T_{31}$ is approximately zero at $a / \lambda=0.384$ for $\theta=60^{\circ}$, while for $\theta=90^{\circ}$, it is nearly one. 


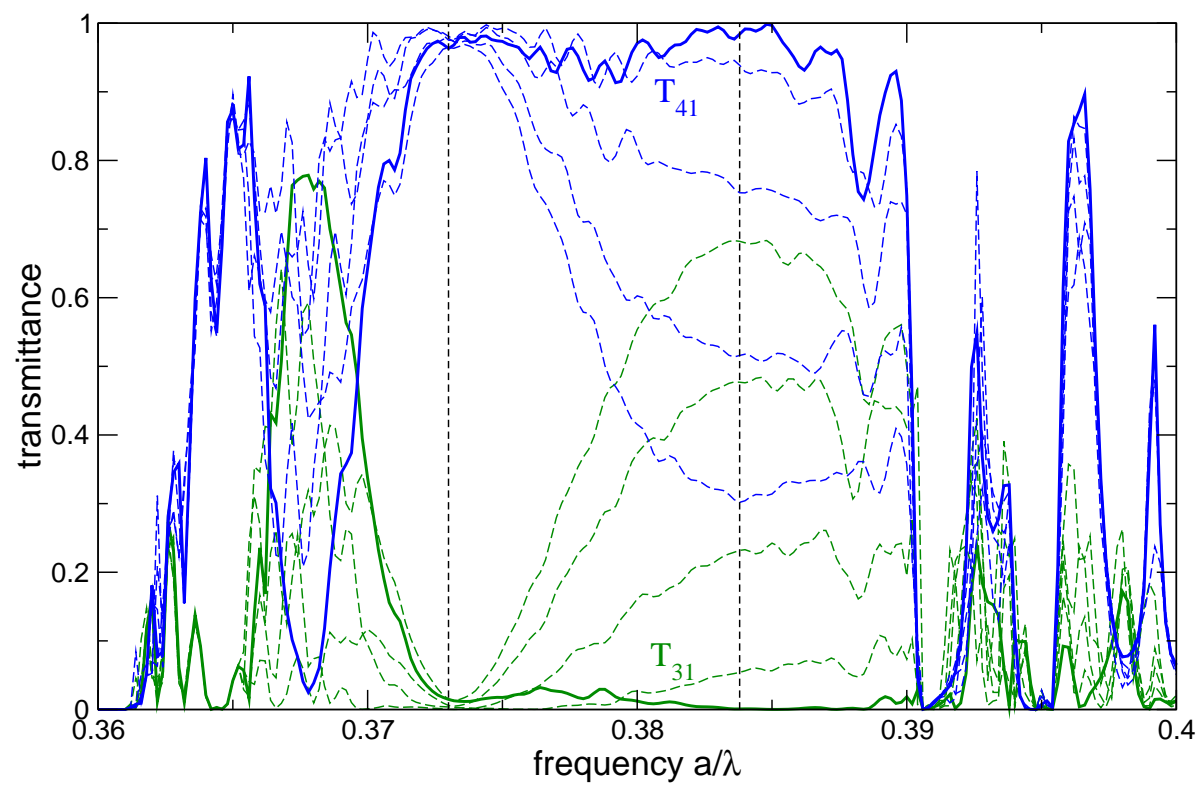

Figure 5.20.: Transmittance spectrum for the actively tunable MZI with a director angle $\theta=90^{\circ}$ in the liquid-crystal delay line with length $L_{\mathrm{D}}=25 a$ (solid curve). The relative phase shift is $\Delta \phi=\pi$ between the two arms of the MZI at the frequency $a / \lambda=0.384$. For shorter delay lines $L_{\mathrm{D}}=6 a, 10 a, 15 a, 20 a$ (dashed lines), $\Delta \phi$ takes values $0<\Delta \phi<\pi$ at $a / \lambda=0.384$. This case is comparable to the unbalanced MZI discussed in Fig. 5.15.

In conclusion, we have designed an MZI that can be switched from the cross state $\left(T_{31} \approx 0.0\right)$ to the bar state $\left(T_{31} \approx 1.0\right)$ by tuning the LC director angle $\theta$ by means of an external electric field. This design could serve as a starting point for a miniaturized switch or modulator device in wavelength-division multiplexing applications. Of course, the result can be further optimized. 


\section{Summary and Conclusions}

A key requirement in the development and optimization of nanophotonic structures is the availability of powerful computational methods. In this thesis, I have presented a guided-mode scattering-matrix approach for the efficient treatment of photonic crystal circuits (published in [69]). The approach relies on the efficient calculation of scattering matrices of basic functional elements via the Wannier-function technique [70]. Fields within a functional element are expanded into photonic Wannier functions. I have discussed in detail the accuracy and convergence of the Wannierfunction expansion and demonstrated the applicability of the approach to large-scale circuits.

The technical and conceptual framework has been given in chapters 2 and 3. In chapter 2, basic matrix equations have been derived for the treatment of cavities, waveguides and functional elements using a Wannier-function expansion basis. The main point is that, ideally, only a few Wannier functions per lattice site are needed to obtain converged results. Furthermore, overlap matrix elements of Wannier functions can be precomputed and reused for the characterization of any defect constellation in a host photonic crystal. This is what makes the Wannier-function expansion very efficient.

The general concept of scattering matrices (S-matrices) has then been introduced in chapter 3. A functional element (FE) is fully characterized by its S-matrix, which contains information about amplitudes and phases of scattered fields. In the present case, the incoming and outgoing fields are guided modes of waveguides connected to the ports of the FE. A circuit, which is a composition of connected FEs, can again be described by an S-matrix. While the S-matrices of FEs are computed on a "microscopic" level using Wannier functions, the circuit S-matrix is constructed by combining those S-matrices. In this procedure, it is of great importance that fundamental symmetries such as reciprocity and congruences of FEs are taken into account. It is then possible to minimize the number of individual FE S-matrices that have to be computed on the costly "microscopic" level. The combination of S-matrices is extremely fast, since it includes only matrix elements of propagating guided modes, resulting in S-matrices of very small size.

A significant part of this work has been the investigation of the accuracy and convergence of the Wannier-function expansion for some representative test cases. In chapter 4, two model systems have been chosen, in which cavity modes and waveguide dispersions have been calculated with the Wannier-function method and checked against reference solutions computed with the MIT Photonic Bands package [63]. Based on the results, I arrive at the following conclusions. Firstly, the Wannierfunction expansion works nicely in some cases, in which only very few Wannier func- 
tions per lattice site are necessary to get converged results. For instance, defect rods in model system 1 in which the permittivity is changed require only six Wannier functions per lattice site. In model system 2, weak defects, i.e., pores filled with a low-index material, can be accurately handled with twenty-six Wannier functions. Secondly, there exist defects for which the Wannier-function expansion shows very slow convergence with the number of Wannier functions. For radius defects in model system 1, it has been observed that the twenty-three available Wannier functions for this system were not sufficient to get quantitatively correct results. Similarly, strong defects in model system 2 , i.e., non-etched pores can not be quantitatively described by the thirty-eight available Wannier functions for this system. The third conclusion is that auxiliary Wannier functions, i.e., Wannier functions computed for a complementary photonic crystal, can help to improve the accuracy of the Wannier-function expansion. For instance, the results for radius defects in model system 1 could be improved significantly. Although the additional basis functions destroy some desirable features of the original Wannier-function basis, such as the orthonormality and the information about band structure, they provide a shortcut to a more complete basis for a specific set of defects in a specific host material. However, they should be applied with care to avoid spurious modes.

In the last part, chapter 5 , the Wannier-function based scattering-matrix approach has then been applied to design an actively-tunable Mach-Zehnder interferometer in a photonic crystal. The main purpose of this part was to demonstrate that with this approach, it is indeed possible to design large-scale circuits in photonic crystals very efficiently. Starting from basic components such as waveguides and optimized bends, successively more complex circuits have been composed. For the directional coupler (DC) presented next, it is apparent that the scattering-matrix approach allows to efficiently handle circuits in which intermediate straight waveguide sections are arbitrarily long. The Mach-Zehnder interferometer (MZI) design based on two connected DCs involves three freely adjustable lengths, including the length of a delay line incorporated into one arm of the MZI. Finally, an actively tunable version of the MZI has been considered by means of filling the pores in the delay line with liquid crystals. The phase shift in the delay line can be designed such that, for one specific frequency, the MZI transmits all light into one output port for one orientation of the liquid-crystal directors, while for another orientation, all light goes into the other output port. Therefore, the resulting device might be used as a switch or a modulator.

Large-scale nanostructured circuits as presented in chapter 5 are very difficult to treat with standard all-purpose techniques such as Finite-Difference Time-Domain (FDTD) [66] or Finite Elements [67] due to the computational expenses they require. The scattering-matrix method based on Wannier functions fills this gap and provides a powerful tool to overcome the limitations of the all-purpose techniques. Clearly, the presented method will not supersede FDTD or Finite Elements for arbitrarily structured media. Rather, it is an alternative approach in the field of photonic crystal based structures. However, since the scattering-matrix approach is not limited to the use of Wannier functions to obtain S-matrices of individual functional elements, it 
could be applied to photonic wire circuits [6] as well. The main requirement for the computation of the S-matrices of individual functional elements is the correct treatment of the phase of the involved fields.

The present work gives rise to a number of new questions and future prospects. Concerning the Wannier-function expansion, further investigation of the use of auxiliary Wannier functions is necessary. It would be desirable to work out the problem of slow convergence with the number of original Wannier functions in greater detail. Unfortunately, the generation of Wannier functions is highly complex [82], so the number of available Wannier functions is limited in practice. Another interesting project would be the formulation and implementation of a time-domain method using Wannier functions as an expansion basis for the fields. This would allow to investigate nonlinear circuits, for instance. Furthermore, in the area of wavelength division multiplexing/demultiplexing (WDM) [100], there are interesting concepts for the wavelength flattening of the device performance [101-103], which could be investigated using photonic crystal circuits. Last but not least, the application of the method to circuits in 3D photonic crystals and 2D photonic crystal slabs is certainly another interesting project. 


\section{A. Model System 1}

In this appendix, we present data for model system 1. This is a square lattice of silicon rods with radius $r=0.18 a$. E-polarized radiation is considered. Figs. A.2 and A.3 show the band structure and the twenty-three Wannier functions, respectively. The Wannier functions were generated according to [82] using the band groupings and frequency windows listed in table A.1.

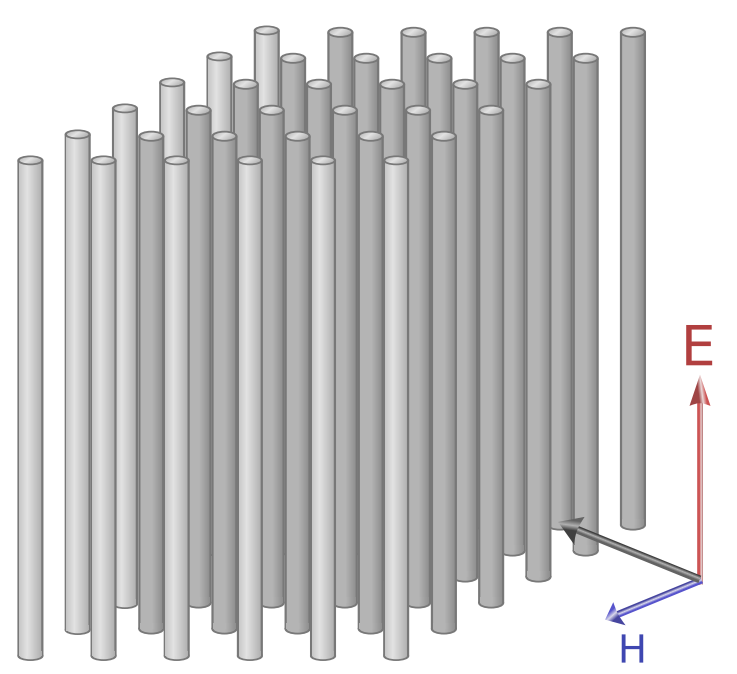

Figure A.1.: Square lattice of silicon rods with radius $r=0.18 a$ in $E$-polarization.

\begin{tabular}{c|c|c} 
bands & inner frequency window & outer frequency window \\
\hline 1 & - & - \\
$2-4$ & - & - \\
$5-9$ & 0.947 & 1.365 \\
$10-16$ & 1.390 & 1.750 \\
$17-23$ & 1.750 & 2.100
\end{tabular}

Table A.1.: Band groupings for the construction. 


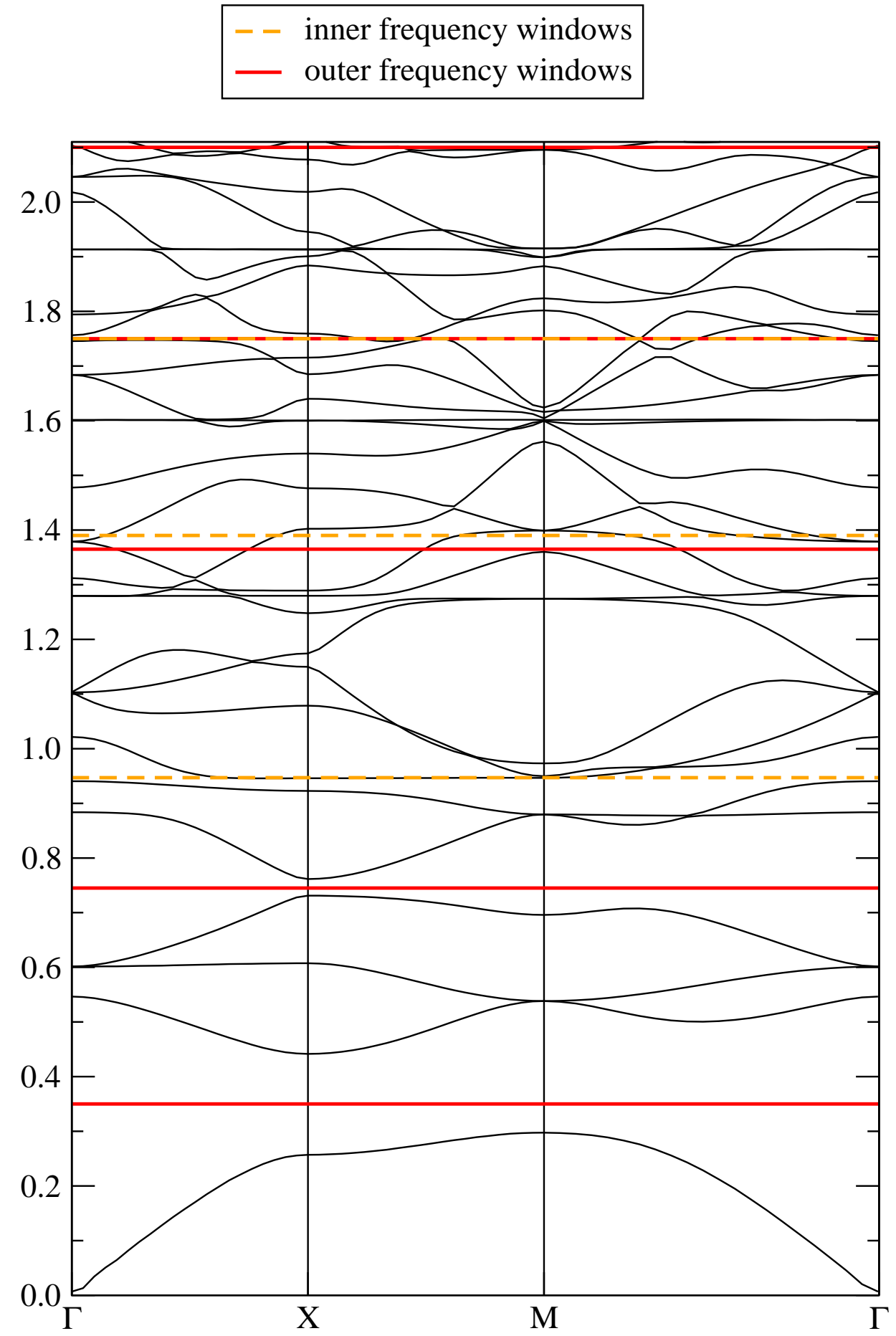

Figure A.2.: Band structure of model system 1. The inner and outer frequency windows are depicted by horizontal lines. Band 1 and bands 2-4 are treated as isolated band groups. 


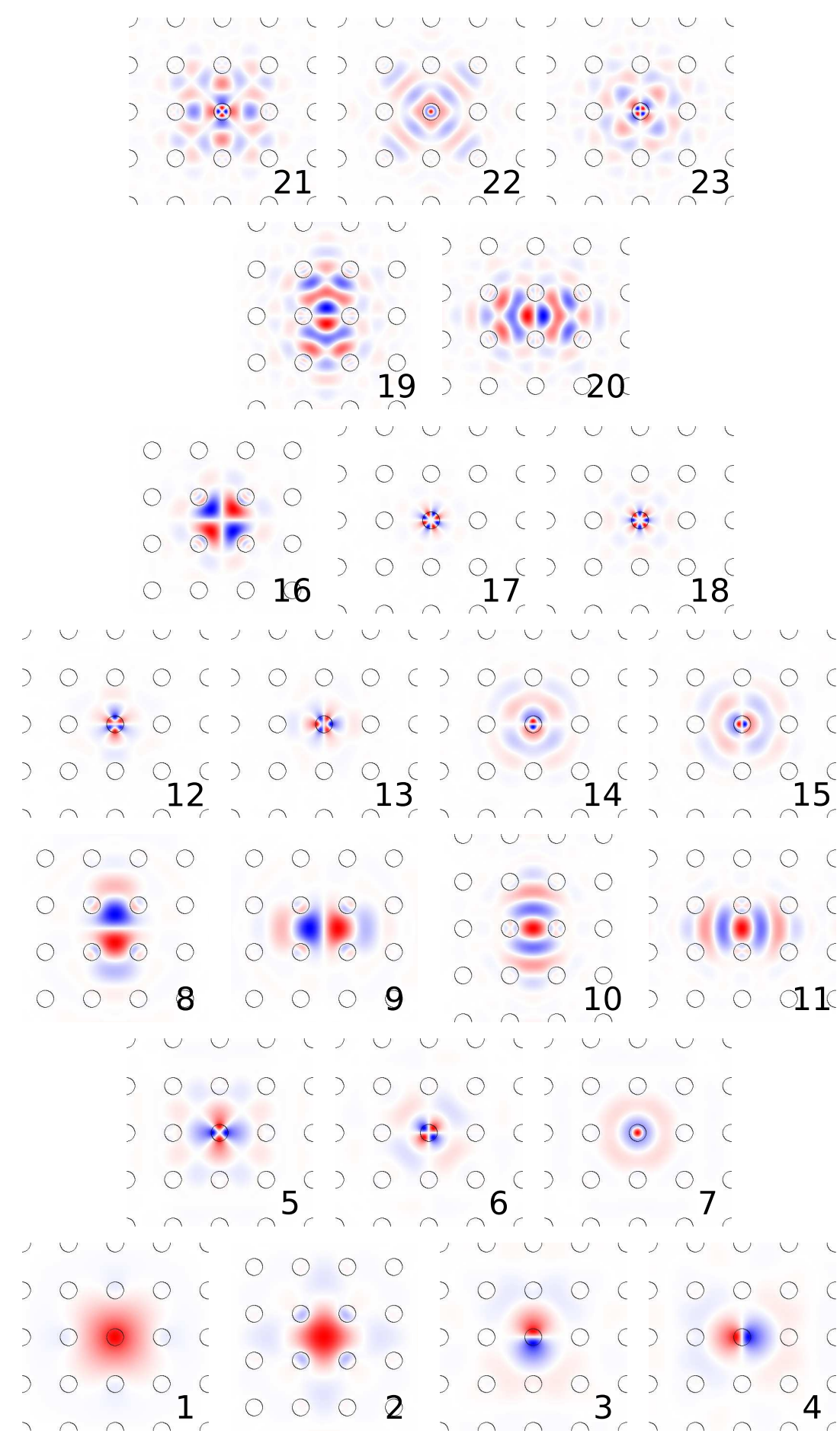

Figure A.3.: Twenty-three Wannier functions of model system 1. 
A. Model System 1 


\section{B. Model System 2}

In this appendix, we present data for model system 2. This is a triangular lattice of air holes with radius $r=0.45 a$ in silicon. $H$-polarized radiation is considered. Figs. B.2 and B.3 show the band structure and the thirty-eight Wannier functions, respectively. The Wannier functions are the ones generated in [82] using the band groupings and frequency windows listed in table B.1.

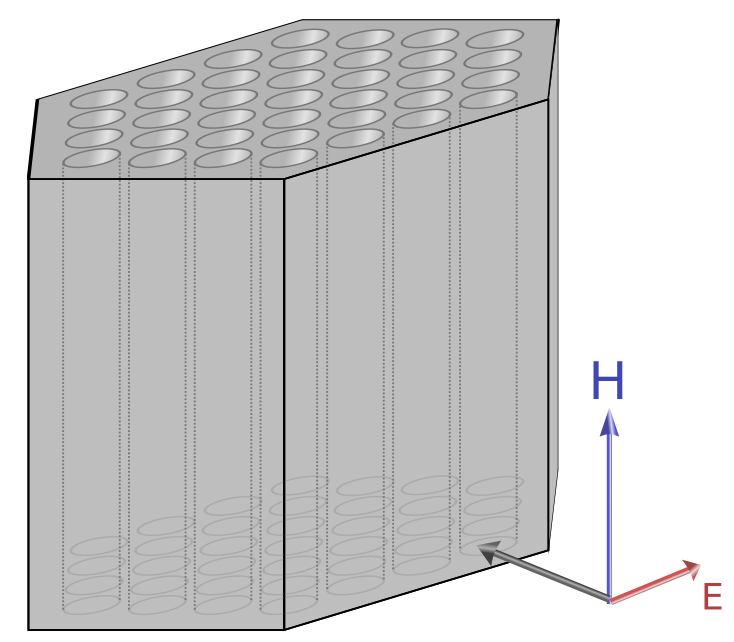

Figure B.1.: Triangular lattice of air holes with radius $r=0.45 a$ in silicon. with $H$-polarized light.

\begin{tabular}{c|c|c} 
bands & inner frequency window & outer frequency window \\
\hline 1 & - & - \\
$2-9$ & 0.88 & 1.12 \\
$10-17$ & 1.30 & 1.49 \\
$18-26$ & 1.6 & 1.715 \\
$27-38$ & - & -
\end{tabular}

Table B.1.: Band groupings for the construction according to [82]. 


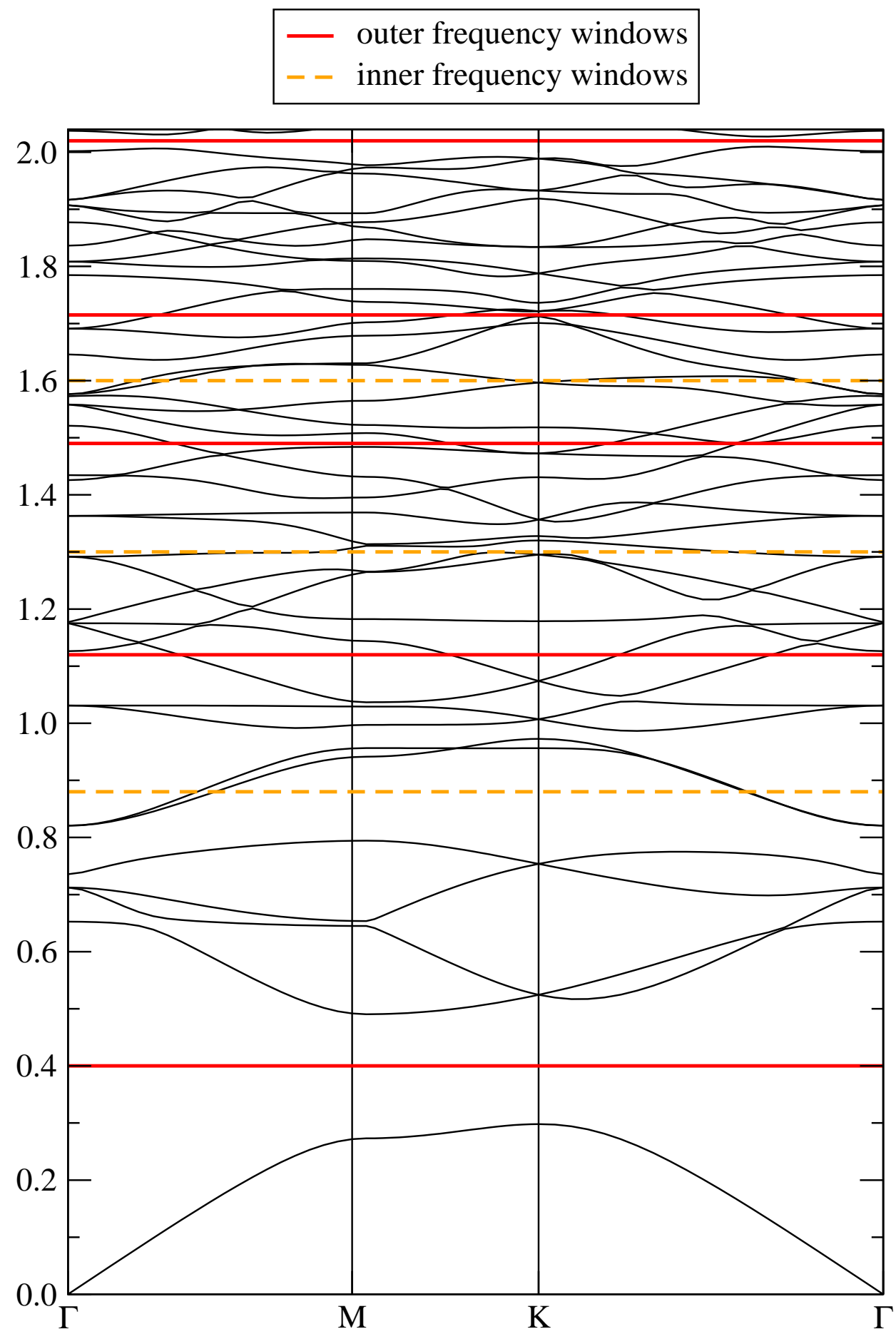

Figure B.2.: Band structure of model system 2. The inner and outer frequency windows are depicted by horizontal lines. Band 1 is treated as an isolated band. 


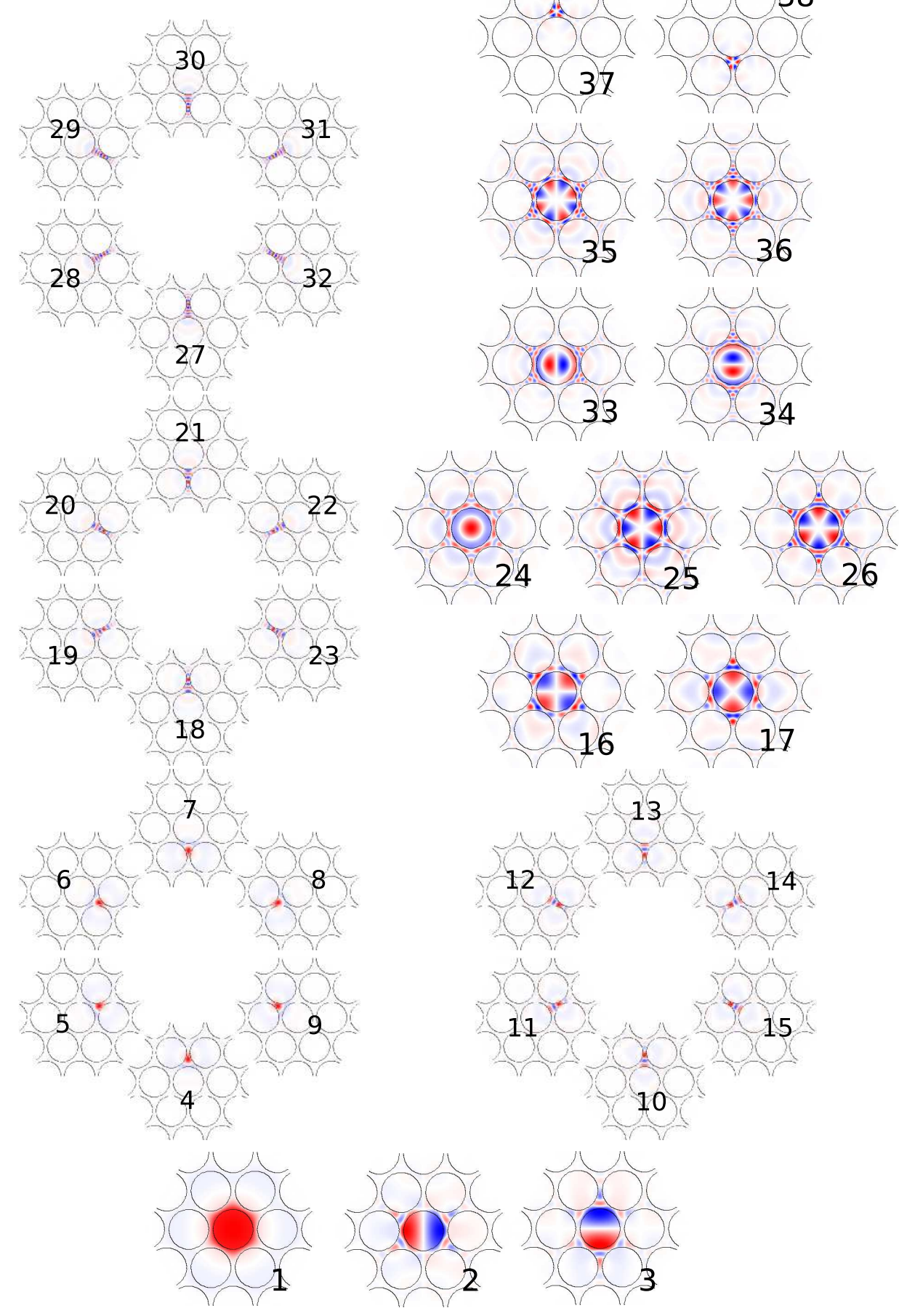

Figure B.3.: Thirty-eight Wannier functions of model system 2. [82] 
B. Model System 2 


\section{Acronyms}

1D one-dimensional

2D two-dimensional

3D three-dimensional

BZ (first) Brillouin zone

DC directional coupler

FE functional element

FDTD finite-difference time-domain

FEM finite element method

LC liquid crystal

LCAO linear combination of atomic orbitals

MPB MIT photonic bands package

MZI Mach-Zehnder interferometer

PhC photonic crystal

PBG photonic band gap

PDE partial differential equation

PWM plane wave expansion method

TE transverse electric

TM transverse magnetic

WDM wavelength division multiplexing/demultiplexing

WF Wannier function

WSC Wigner-Seitz cell 


\section{Bibliography}

[1] http://tenyears-www.web.cern.ch/ (2003).

[2] Source: BITKOM (http://www.bitkom.org/) (2008).

[3] W. Bogaerts. Nanophotonic Waveguides and Photonic Crystals in Silicon-onInsulator. Ph.D. thesis, Universiteit Gent, Belgium (2003).

[4] W. Bogaerts, R. Baets, P. Dumon, V. Wiaux, S. Beckx, D. Taillaert, B. Luyssaert, J. Van Campenhout, P. Bienstman, and D. Van Thourhout. Nanophotonic waveguides in silicon-on-insulator fabricated with CMOS technology. J. Lightwave Technol. 23 (1), 401 (2005).

[5] Y. Hibino. Silica-based planar lightwave circuits and their applications. MRS Bulletin 28 (5), 365 (2003).

[6] W. Bogaerts, D. Taillaert, B. Luyssaert, P. Dumon, J. Van Campenhout, P. Bienstman, D. Van Thourhout, R. Baets, V. Wiaux, and S. Beckx. Basic structures for photonic integrated circuits in silicon-on-insulator. Opt. Express 12 (8), 1583 (2004).

[7] R. De La Rue, H. Chong, M. Gnan, N. Johnson, I. Ntakis, P. Pottier, M. Sorel, A. M. Zain, H. Zhang, E. Camargo, C. Jin, M. Armenise, and C. Ciminelli. Photonic crystal and photonic wire nano-photonics based on silicon-on-insulator. New J. Phys. 8 (10), 256 (2006).

[8] Y. Vlasov and S. McNab. Losses in single-mode silicon-on-insulator strip waveguides and bends. Opt. Express 12 (8), 1622 (2004).

[9] H. Yamada, T. Chu, S. Ishida, and Y. Arakawa. Si photonic wire waveguide devices. IEEE J. Sel. Top. Quantum Electron. 12 (6), 1371 (2006).

[10] S. John, O. Toader, and K. Busch. Photonic band gap materials: a semiconductor for light. In R. A. Meyers, ed., Encyclopedia of Physical Science and Technology, vol. 12, 3rd ed. (Academic Press, 2001).

[11] J. D. Joannopoulos, R. D. Meade, and J. N. Winn. Photonic Crystals: Molding The Flow of Light (Princeton University Press, 1995).

[12] E. Yablonovitch. Photonic band-gap structures. J. Opt. Soc. Am. B 10 (2), 283 (1993). 
[13] K. Busch, G. von Freymann, S. Linden, S. F. Mingaleev, L. Tkeshelashvili, and M. Wegener. Periodic nanostructures for photonics. Phys. Rep. 444 (3-6), 101 (2007).

[14] S. Noda. Recent progresses and future prospects of two- and three-dimensional photonic crystals. J. Lightwave Technol. 24 (12), 4554 (2006).

[15] M. Notomi, K. Yamada, A. Shinya, J. Takahashi, C. Takahashi, and I. Yokohama. Extremely large group-velocity dispersion of line-defect waveguides in photonic crystal slabs. Phys. Rev. Lett. 87 (25), 253902 (2001).

[16] Y. A. Vlasov, M. O'Boyle, H. F. Hamann, and S. J. McNab. Active control of slow light on a chip with photonic crystal waveguides. Nature 438 (7064), 65 (2005).

[17] M. F. Yanik and S. Fan. Stopping light all optically. Phys. Rev. Lett. 92 (8), 083901 (2004).

[18] H. Kosaka, T. Kawashima, A. Tomita, M. Notomi, T. Tamamura, T. Sato, and S. Kawakami. Superprism phenomena in photonic crystals. Phys. Rev. B 58 (16), R10096 (1998).

[19] C. Luo, S. G. Johnson, J. D. Joannopoulos, and J. B. Pendry. All-angle negative refraction without negative effective index. Phys. Rev. B 65 (20), 201104 (2002).

[20] M. Notomi. Theory of light propagation in strongly modulated photonic crystals: Refractionlike behavior in the vicinity of the photonic band gap. Phys. Rev. B 62 (16), 10696 (2000).

[21] S. John and J. Wang. Quantum electrodynamics near a photonic band gap: Photon bound states and dressed atoms. Phys. Rev. Lett. 64 (20), 2418 (1990).

[22] S. John and T. Quang. Resonant nonlinear dielectric response in a photonic band gap material. Phys. Rev. Lett. 76 (14), 2484 (1996).

[23] E. Yablonovitch. Inhibited spontaneous emission in solid-state physics and electronics. Phys. Rev. Lett. 58 (20), 2059 (1987).

[24] S. John. Strong localization of photons in certain disordered dielectric superlattices. Phys. Rev. Lett. 58 (23), 2486 (1987).

[25] I. Fushman, D. Englund, A. Faraon, N. Stoltz, P. Petroff, and J. Vučković. Controlled phase shifts with a single quantum dot. Science 320 (5877), 769 (2008).

[26] T. F. Krauss, R. M. De La Rue, and S. Brand. Two-dimensional photonicbandgap structures operating at near infrared wavelengths. Nature 383 (6602), 699 (1996). 
[27] T. F. Krauss and R. M. De La Rue. Photonic crystals in the optical regime past, present and future. Prog. Quantum Electron. 23 (2), 51 (1999).

[28] A. Birner, R. B. Wehrspohn, U. M. Gösele, and K. Busch. Silicon-based photonic crystals. Adv. Mater. 13 (6), 377 (2001).

[29] A. C. Edrington, A. M. Urbas, P. DeRege, C. X. Chen, T. M. Swager, N. Hadjichristidis, M. Xenidou, L. J. Fetters, J. D. Joannopoulos, Y. Fink, and E. L. Thomas. Polymer-based photonic crystals. Adv. Mater. 13 (6), 421 (2001).

[30] S. Noda, M. Imada, A. Chutinan, and N. Yamamoto. III-V based-semiconductor photonic crystals. Opt. Quantum Electron. 34 (8), 723 (2002).

[31] S. Lin, J. Fleming, D. Hetherington, B. Smith, R. Biswas, K. Ho, M. Sigalas, W. Zubrzycki, S. Kurtz, and J. Bur. A three-dimensional photonic crystal operating at infrared wavelengths. Nature 394 (6690), 251 (1998).

[32] J. E. G. J. Wijnhoven and W. L. Vos. Preparation of photonic crystals made of air spheres in titania. Science 281 (5378), 802 (1998).

[33] S. Noda, K. Tomoda, N. Yamamoto, and A. Chutinan. Full three-dimensional photonic bandgap crystals at near-infrared wavelengths. Science 289 (5479), 604 (2000).

[34] A. Blanco, E. Chomski, S. Grabtchak, M. Ibisate, S. John, S. W. Leonard, C. Lopez, F. Meseguer, H. Miguez, J. P. Mondia, G. A. Ozin, O. Toader, and H. M. van Driel. Large-scale synthesis of a silicon photonic crystal with a complete three-dimensional bandgap near 1.5 micrometres. Nature 405 (6785), 437 (2000).

[35] M. Campbell, D. Sharp, M. Harrison, R. Denning, and A. Turberfield. Fabrication of photonic crystals for the visible spectrum by holographic lithography. Nature 404 (6773), 53 (2000).

[36] Y. V. Miklyaev, D. C. Meisel, A. Blanco, G. von Freymann, K. Busch, W. Koch, C. Enkrich, M. Deubel, and M. Wegener. Three-dimensional face-centered-cubic photonic crystal templates by laser holography: fabrication, optical characterization, and band-structure calculations. Appl. Phys. Lett. 82 (8), 1284 (2003).

[37] H.-B. Sun, S. Matsuo, and H. Misawa. Three-dimensional photonic crystal structures achieved with two-photon-absorption photopolymerization of resin. Appl. Phys. Lett. 74 (6), 786 (1999).

[38] M. Straub and M. Gu. Near-infrared photonic crystals with higher-order bandgaps generated by two-photon photopolymerization. Opt. Lett. 27 (20), 1824 (2002). 
[39] M. Deubel, G. von Freymann, M. Wegener, S. Pereira, K. Busch, and C. Soukoulis. Direct laser writing of three-dimensional photonic-crystal templates for telecommunications. Nat. Mater. 3 (7), 444 (2004).

[40] S. Wong, M. Deubel, F. Pérez-Willard, S. John, G. A. Ozin, M. Wegener, and G. von Freymann. Direct laser writing of three-dimensional photonic crystals with complete a photonic bandgap in chalcogenide glasses. Adv. Mater. 18 (3), 265 (2006).

[41] N. Tétreault, G. von Freymann, M. Deubel, M. Hermatschweiler, F. PérezWillard, S. John, M. Wegener, and G. A. Ozin. New route to three-dimensional photonic bandgap materials: Silicon double inversion of polymer templates. Adv. Mater. 18 (4), 457 (2006).

[42] M. Lončar, T. Doll, J. Vučković, and A. Scherer. Design and fabrication of silicon photonic crystal optical waveguides. J. Lightwave Technol. 18 (10), $1402(2000)$.

[43] T. Krauss. Planar photonic crystal waveguide devices for integrated optics. Phys. Status Solidi A 197 (3), 688 (2003).

[44] V. Lehmann. Electrochemistry of Silicon (Wiley-VCH, Weinheim, 2002).

[45] K. Busch, S. Lölkes, R. B. Wehrspohn, and H. Föll, eds. Photonic Crystals: Advances in Design, Fabrication, and Characterization (Wiley-VCH, Weinheim, 2004).

[46] S.-I. Takayama, H. Kitagawa, Y. Tanaka, T. Asano, and S. Noda. Experimental demonstration of complete photonic band gap in two-dimensional photonic crystal slabs. Appl. Phys. Lett. 87 (6), 061107 (2005).

[47] A. Green, E. Istrate, and E. Sargent. Efficient design and optimization of photonic crystal waveguides and couplers: The interface diffraction method. Opt. Express 13 (19), 7304 (2005).

[48] A. Mekis, J. C. Chen, I. Kurland, S. Fan, P. R. Villeneuve, and J. D. Joannopoulos. High transmission through sharp bends in photonic crystal waveguides. Phys. Rev. Lett. 77 (18), 3787 (1996).

[49] A. Chutinan, M. Okano, and S. Noda. Wider bandwidth with high transmission through waveguide bends in two-dimensional photonic crystal slabs. Appl. Phys. Lett. 80 (10), 1698 (2002).

[50] S. F. Mingaleev, M. Schillinger, D. Hermann, and K. Busch. Tunable photonic crystal circuits: concepts and designs based on single-pore infiltration. Opt. Lett. 29 (24), 2858 (2004).

[51] M. Koshiba. Wavelength division multiplexing and demultiplexing with photonic crystal waveguide couplers. J. Lightwave Technol. 19 (12), 1970 (2001). 
[52] S.-H. Jeong, N. Yamamoto, J.-I. Sugisaka, M. Okano, and K. Komori. GaAsbased two-dimensional photonic crystal slab ring resonator consisting of a directional coupler and bent waveguides. J. Opt. Soc. Am. B 24 (8), 1951 (2007).

[53] M. Soljačić, S. G. Johnson, S. Fan, M. Ibanescu, E. Ippen, and J. D. Joannopoulos. Photonic-crystal slow-light enhancement of nonlinear phase sensitivity. J. Opt. Soc. Am. B 19 (9), 2052 (2002).

[54] K. Guven and E. Ozbay. Coupling and phase analysis of cavity structures in two-dimensional photonic crystals. Phys. Rev. B 71 (8), 085108 (2005).

[55] K. Busch and S. John. Liquid-crystal photonic-band-gap materials: The tunable electromagnetic vacuum. Phys. Rev. Lett. 83 (5), 967 (1999).

[56] K. Yoshino, Y. Shimoda, Y. Kawagishi, K. Nakayama, and M. Ozaki. Temperature tuning of the stop band in transmission spectra of liquid-crystal infiltrated synthetic opal as tunable photonic crystal. Appl. Phys. Lett. 75 (7), 932 (1999).

[57] H. Takeda and K. Yoshino. Tunable light propagation in $Y$-shaped waveguides in two-dimensional photonic crystals utilizing liquid crystals as linear defects. Phys. Rev. B 67 (7), 073106 (2003).

[58] T. Yasuda, Y. Tsuji, and M. Koshiba. Tunable light propagation in photonic crystal coupler filled with liquid crystal. IEEE Photonics Technol. Lett. 17 (1), 55 (2005).

[59] K. Busch. Photonic band structure theory: assessment and perspectives. Comptes Rendus Physique 3 (1), 53 (2002).

[60] K. M. Ho, C. T. Chan, and C. M. Soukoulis. Existence of a photonic gap in periodic dielectric structures. Phys. Rev. Lett. 65 (25), 3152 (1990).

[61] R. D. Meade, A. M. Rappe, K. D. Brommer, J. D. Joannopoulos, and O. L. Alerhand. Accurate theoretical analysis of photonic band-gap materials. Phys. Rev. B 48 (11), 8434 (1993).

[62] K. Busch and S. John. Photonic band gap formation in certain self-organizing systems. Phys. Rev. E 58 (3), 3896 (1998).

[63] S. Johnson and J. Joannopoulos. Block-iterative frequency-domain methods for Maxwell's equations in a planewave basis. Opt. Express 8 (3), 173 (2001).

[64] D. Dobson. An efficient method for band structure calculations in $2 D$ photonic crystals. J. Comput. Phys. 149 (2), 363 (1999).

[65] D. Hermann, M. Frank, K. Busch, and P. Wölfle. Photonic band structure computations. Opt. Express 8 (3), 167 (2001). 
[66] A. Taflove and S. C. Hagness. Computational Electrodynamics: The FiniteDifference Time-Domain Method. 3rd ed. (Artech House, 2005).

[67] J. Jin. The Finite Element Method in Electromagnetics (John Wiley \& Sons, 2002).

[68] M. Koshiba, Y. Tsuji, and M. Hikari. Time-domain beam propagation method and its application to photonic crystal circuits. J. Lightwave Technol. 18 (1), $102(2000)$.

[69] D. Hermann, M. Schillinger, S. F. Mingaleev, and K. Busch. Wannier-function based scattering-matrix formalism for photonic crystal circuitry. J. Opt. Soc. Am. B 25 (2), 202 (2008).

[70] K. Busch, S. F. Mingaleev, A. García-Martín, M. Schillinger, and D. Hermann. The Wannier function approach to photonic crystal circuits. J. Phys.: Condens. Matter 15 (30), R1233 (2003).

[71] J. D. Jackson. Classical Electrodynamics. 3rd ed. (Wiley, 1999).

[72] V. Veselago. Electrodynamics of substances with simultaneously negative values of $\sigma$ and $\mu$. Sov. Phys. USPEKHI 10 (4), 509 (1968).

[73] D. R. Smith, J. B. Pendry, and M. C. K. Wiltshire. Metamaterials and negative refractive index. Science 305 (5685), 788 (2004).

[74] J. B. Pendry. Negative refraction makes a perfect lens. Phys. Rev. Lett. 85 (18), 3966 (2000).

[75] T. Ochiai, U. Leonhardt, and J. C. Nacher. A novel design of dielectric perfect invisibility devices. J. Math. Phys. 49 (3), 032903 (2008).

[76] P. Mack. Tunable anisotropic defect structures in 2D photonic crystals using the Wannier function approach. Master's thesis, Universität Karlsruhe, Institut für Theoretische Festkörperphysik (2006).

[77] N. W. Ashcroft and N. D. Mermin. Solid State Physics (Brooks Cole, 1976).

[78] T. Inui, Y. Tanabe, and Y. Onodera. Group theory and its applications in physics (Springer-Verlag, Berlin, 1990).

[79] K. Sakoda. Optical Properties of Photonic Crystals (Springer, Berlin, 2001).

[80] G. H. Wannier. The structure of electronic excitation levels in insulating crystals. Phys. Rev. 52 (3), 191 (1937).

[81] W. Kohn. Analytic properties of Bloch waves and Wannier functions. Phys. Rev. 115 (4), 809 (1959). 
[82] M. Schillinger. Maximally localized photonic Wannier functions for the highly efficient description of integrated Photonic Crystal circuits. Ph.D. thesis, Universität Karlsruhe, Institut für Theoretische Festkörperphysik (2006).

[83] N. Marzari and D. Vanderbilt. Maximally localized generalized Wannier functions for composite energy bands. Phys. Rev. B 56 (20), 12847 (1997).

[84] I. Souza, N. Marzari, and D. Vanderbilt. Maximally localized Wannier functions for entangled energy bands. Phys. Rev. B 65 (3), 035109 (2001).

[85] F. Intonti, S. Vignolini, V. Türck, M. Colocci, P. Bettotti, L. Pavesi, S. L. Schweizer, R. Wehrspohn, and D. Wiersma. Rewritable photonic circuits. Appl. Phys. Lett. 89 (21), 211117 (2006).

[86] E. P. Kosmidou, E. E. Kriezis, and T. D. Tsiboukis. Analysis of tunable photonic crystal directional couplers. J. Appl. Phys. 100 (4), 043118 (2006).

[87] Z. Wang and S. Fan. Optical circulators in two-dimensional magneto-optical photonic crystals. Opt. Lett. 30 (15), 1989 (2005).

[88] S. Cohn and R. Levy. History of microwave passive components with particular attention to directional couplers. IEEE Trans. Microwave Theory Tech. 32 (9), 1046 (1984).

[89] L. Zehnder. Ein neuer Interferenzrefraktor. Zeitschrift für Instrumentenkunde 11 (8), 275 (1891).

[90] L. Mach. Über einen Interferenzrefraktor. Zeitschrift für Instrumentenkunde 12 (3), 89 (1892).

[91] S. Kirkpatrick, C. D. Gelatt, and M. P. Vecchi. Optimization by simulated annealing. Science 220 (4598), 671 (1983).

[92] R. L. Haupt and D. H. Werner. Genetic Algorithms in Electromagnetics (John Wiley \& Sons, 2007).

[93] Numerical Recipes in C: The Art of Scientific Computing (Cambridge University Press, 1992).

[94] A. T. de Hoop. Reciprocity of the electromagnetic field. Appl. Sci. Res. B 8, 135 (1959).

[95] D. S. Saxon. Tensor scattering matrix for the electromagnetic field. Phys. Rev. 100 (6), 1771 (1955).

[96] R. Carminati, J. J. Sáenz, J.-J. Greffet, and M. Nieto-Vesperinas. Reciprocity, unitarity, and time-reversal symmetry of the $S$ matrix of fields containing evanescent components. Phys. Rev. A 62 (1), 012712 (2000). 
[97] R. J. Potton. Reciprocity in optics. Rep. Prog. Phys. 67 (5), 717 (2004).

[98] E. Istrate and E. H. Sargent. Photonic crystal heterostructures and interfaces. Rev. Mod. Phys. 78 (2), 455 (2006).

[99] Y. Jiao, S. Fan, and D. A. B. Miller. Demonstration of systematic photonic crystal device design and optimization by low-rank adjustments: an extremely compact mode separator. Opt. Lett. 30 (2), 141 (2005).

[100] H. J. R. Dutton. Understanding Optical Communications. 1st ed. (IBM International Technical Support Organization (http://www.redbooks.ibm.com), 1998).

[101] M. Cherchi. Wavelength-flattened directional couplers: A geometrical approach. Appl. Opt. 42 (36), 7141 (2003).

[102] M. Cherchi. Design scheme for Mach-Zehnder interferometric coarse wavelength division multiplexing splitters and combiners. J. Opt. Soc. Am. B 23 (9), 1752 (2006).

[103] K. Jinguji, N. Takato, Y. Hida, T. Kitoh, and M. Kawachi. Two-port optical wavelength circuits composed of cascaded Mach-Zehnder interferometers with point-symmetrical configurations. J. Lightwave Technol. 14 (10), 2301 (1996). 


\section{Danksagung}

$\mathrm{Zu}$ guter Letzt möchte ich an dieser Stelle allen Personen danken, die direkt oder indirekt zum Entstehen dieser Arbeit beigetragen haben.

Zunächst bedanke ich mich ganz herzlich bei Prof. Dr. Kurt Busch für die interessante Themenstellung und seine jahrelange Unterstützung, auch in Zeiten, wo mir die Computeradministration wenig Zeit für Physik ließ. Die anregenden Diskussionen mit ihm waren immer ein Quell neuer Motivation und Ideen, ohne die diese Arbeit nicht möglich gewesen wäre. Er hat mir immer genug Freiraum gelassen, um eigene Vorstellungen zu verwirklichen.

Prof. Dr. Peter Wölfle danke ich für die Möglichkeit, meine Promotion am Institut für Theorie der Kondensierten Materie zu beginnen und für die Übernahme des Korreferats.

Ein besonderer Dank gilt den Mitgliedern der "Photonics Group", die ich von Beginn an kennen und schätzen gelernt habe. I thank Dr. Antonio García-Martín for his friendly style at work and personally, Dr. Sergei Mingaleev for providing the code basis upon which I could build and for valuable discussions, as well as Dr. Lasha Tkeshelashvili for many nightly conversations on physics, not only in the final preparation stage of this work. Ganz besonders danke ich meinen langjährigen Weggefährten und Zimmerkollegen, Dr. Marcus Diem, Dr. Meikel Frank und Dr. Matthias Schillinger. Es war wunderbar, mit Euch das Zimmer zu teilen. Gerne erinnere ich mich auch an die spontanen Grillparties auf der Chemieterrasse. Auch allen nachfolgenden Generationen von Diplomanden und Doktoranden möchte ich für die angenehme Arbeitsatmosphäre und die zahlreichen geselligen Zusammenkünfte danken. Außerdem hätte ich die Zeit des Zusammenschreibens ohne das Süßigkeitendoping von Dipl.-Phys. Christian Wolff vermutlich nicht überstanden.

Bei Dr. Lasha Tkeshelashvili, Dipl.-Phys. Christian Wolff, Dipl.-Phys. Jens Niegemann, Dipl.-Phys. Patrick Mack, Dipl.-Phys. Sabine Essig und Christopher Kölper bedanke ich mich außerdem für das kritische Korrekturlesen dieser Arbeit.

Nicht vergessen möchte ich an dieser Stelle auch meine Kollegen Dr. Andreas Poenicke und Dipl.-Phys. Tobias Ulbricht, mit denen ich so manches Computerproblem für die beiden Institute TKM und TFP zu lösen hatte. Auch wenn es manchmal stressig war, ich hab' immer gerne mit Euch zusammengearbeitet.

Abschließend möchte ich meinen Eltern ganz herzlich für die stete Unterstützung danken, ohne die das alles nicht möglich gewesen wäre. 